\title{
Modelling and Stress Analysis of a \\ Polyurethane Diaphragm for use in \\ Artificial Hearts
}

by

Shamir Doshi, B.A.Sc.

University of Ottawa

A thesis submitted to

the Faculty of Graduate Studies and Research

in partial fulfillment of

the requirements for the degree of

Masters of Applied Science

Ottawa-Carleton Institute for

Mechanical and Aerospace Engineering

Department of

Mechanical and Aerospace Engineering

Carleton University

Ottawa, Ontario

Dec 22, 2003

(c) Copyright 2003 


\author{
National Library \\ of Canada \\ Acquisitions and \\ Bibliographic Services \\ 395 Wellington Street \\ Ottawa ON K1A ON4 \\ Canada
}

Bibliothèque nationale

du Canada

Acquisisitons et services bibliographiques

395 , rue Wellington Ottawa ON K1A ON4 Canada
Your file Votre référence ISBN: 0-612-93934-0

Ourfile Notre référence ISBN: 0-612-93934-0
The author has granted a nonexclusive licence allowing the National Library of Canada to reproduce, loan, distribute or sell copies of this thesis in microform, paper or electronic formats.

The author retains ownership of the copyright in this thesis. Neither the thesis nor substantial extracts from it may be printed or otherwise reproduced without the author's permission.
L'auteur a accordé une licence non exclusive permettant à la Bibliothèque nationale du Canada de reproduire, prêter, distribuer ou vendre des copies de cette thèse sous la forme de microfiche/film, de reproduction sur papier ou sur format électronique.

L'auteur conserve la propriété du droit d'auteur qui protège cette thèse. $\mathrm{Ni}$ la thèse ni des extraits substantiels de celle-ci ne doivent être imprimés ou aturement reproduits sans son autorisation.
In compliance with the Canadian Privacy Act some supporting forms may have been removed from this dissertation.

While these forms may be included in the document page count, their removal does not represent any loss of content from the dissertation.
Conformément à la loi canadienne sur la protection de la vie privée, quelques formulaires secondaires ont été enlevés de ce manuscrit.

Bien que ces formulaires aient inclus dans la pagination, il n'y aura aucun contenu manquant. 
PAGINATION ERROR.

TEXI COMPLETE.
ERREOR DE PAGINATION.

LE TEXTE EST COMPLET. 


\section{Abstract}

Artificial heart polyurethane diaphragms are subjected to cyclic loading which makes devices scheduled for long-term use prone to fatigue failure. Stress concentrations developed during the deformation of the diaphragm are the sources of microcracks that lead to the untimely fatigue failure. A solid model of a typical diaphragm shape was analyzed for stress and strain using Finite Element Analysis (FEA)software, ABAQUS/Standard. The Mooney-Rivlin, hyperelastic, strain energy model for strains $<50 \%$, is evaluated for use in the FEA analysis. The material constants for the strain energy model can be determined from standard homogenous material tests, uniaxial, biaxial and planar materials tests. For this study, the material constants were determined from uniaxial stress-strain data and biaxial stress-strain data for biomedical grade polyurethane. Uniaxial data was provided from a local manufacturer of the polyurethane material. For biaxial data, an inflation apparatus was created to determine the biaxial stress-strain curve for the same material. Planar test data was excluded from the analysis. The results from both data sets were evaluated for use with the Mooney-Rivlin curve and are compared against published data for Biomer. A step-by-step analysis of ABAQUS/Standard was undertaken to assess boundary conditions, analysis methods and element types for 3D analysis of the polyurethane diaphragm. A structured hexagonal mesh using second order, hybrid, reduced integration elements, C3D20RH and a built-in boundary condition is recommended for use in the diaphragm inversion and deformation analysis. The model is expected to form the basis of future efforts investigating more complex shapes. This study found strains over $50 \%$ and stresses over 5MPa appear near the periphery of the model when the diaphragm is inverted and pressurized to $30 \mathrm{KPa}(255 \mathrm{mmHg})$. 


\section{Acknowledgements}

I would like to thank my supervisor and friend, Dr. Robert Bell, for the patience, the clarity and the encouragement over the years.

Thank you to Dr. John Goldak for his time in answering my many, many questions in the area of FEA.

I am grateful to Jim Sliwka and Alex Proctor of the Mechanical and Aerospace Machine Shop who, rather than making the inflation apparatus for me, taught me how to use the milling machine and lathe so that I could make it myself; an even better arrangement.

Special thanks go to the biomedical companies and researchers that supported this work through the contribution of data and materials. Dr. Tofy Mussivand for his support and encouragement. Thank you to Dr. Carl McMillin for all the contact information and for answering my questions on fatigue. To Robert Ward and Jim Serpillio for their contribution. I am also grateful to Dr. John Kardos from the University of Washington, for providing me the Biomer data.

I thank my Mom and Dad and sister, Sheema who encouraged me to begin Masters studies and have always supported me with important advice and encouragement.

Finally, I am thankful to my wife, Jayna, my source of strength and love. 


\section{Contents}

Acceptance $\quad$ ii

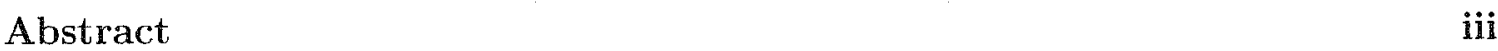

Acknowledgements $\quad$ iv

Contents $\quad$ v

List of Figures $\quad$ xii

List of Tables $\quad$ xvi

1 Introduction 1

1.1 Background . . . . . . . . . . . . . . . . . . 1

1.2 Thesis Objectives ........................... 5

1.3 Thesis Overview . . . . . . . . . . . . . . . . . . . . 6

2 Literature Review $\quad 9$

2.1 LVAD Arrangement and Function . . . . . . . . . . . . . . . . . 9

2.2 Diaphragm Requirements . . . . . . . . . . . . . . . . . . . 13

2.3 Pressure Development Behind the SAC and Deformation . . . . . . . . 14

2.4 Mechanical model . . . . . . . . . . . . . . . . . . . . 18 
2.5. Finite Element Analysis of Diaphragms . . . . . . . . . . . . . . . . . 19

2.6 Photoelastic Analysis . . . . . . . . . . . . . . . . 20

2.7 Fatigue Analysis . . . . . . . . . . . . . . . . . . 22

2.8 Early Efforts in Diaphragm Design . . . . . . . . . . . . . . 24

3 Molecular Theory of Polyurethane $\quad 29$

3.1 Thermoplastic Polyurethane . . . . . . . . . . . . . . . . 29

3.2 Elastomers Background . . . . . . . . . . . . . . . . . . 30

3.3 Polyurethane Microstructure . . . . . . . . . . . . . . . . 33

3.4 Polyurethane Macrostructure . . . . . . . . . . . . . . . . . 34

3.5 Molecular Interaction During Stress . . . . . . . . . . . . . . 35

3.6 Molecular Theory of Rubber Elasticity . . . . . . . . . . . . . . 36

4 Finite Deformation Elasticity $\quad 39$

4.1 Measures of Strain . . . . . . . . . . . . . . . . 40

4.2 Measures of Stress . . . . . . . . . . . . . . . . . 44

4.3 Equilibrium Laws of Continuum Mechanics . . . . . . . . . . . . 46

4.3 .1 Conservation of Mass . . . . . . . . . . . . . 46

4.3 .2 Conservation of Linear Momentum . . . . . . . . . . . . . 47

4.3 .3 Conservation of Angular Momentum . . . . . . . . . . . . 48

4.3.4 Principle of Virtual Work (Conservation of Energy) . . . . . . . . 48

4.4 Neumann and Dirichlet Boundary Conditions . . . . . . . . . . . . 49

4.5 Constitutive Equation . . . . . . . . . . . . . . . 50

4.6 Application to Diaphragm Design . . . . . . . . . . . . . 56

4.6.1 Other Considerations . . . . . . . . . . . . . 58

4.7 Material Properties and Coefficients . . . . . . . . . . . . . 60

4.7 .1 Uniaxial Tensile Test . . . . . . . . . . . . . . . 61 
4.7 .2 Biaxial Tensile Test . . . . . . . . . . . . . . 71

4.7.3 Calculation of Mooney-Rivlin Coefficients . . . . . . . . . 78

5 Finite Element Formulation $\quad 87$

5.1 Incompressibility constraint . . . . . . . . . . . 88

5.2 Numerical Procedures . . . . . . . . . . . . . . . . . . . 90

5.2 .1 Newton Approximation . . . . . . . . . . . . . . . . . . 90

5.2 .2 Arc-Length Method . . . . . . . . . . . . . . . . . . . . 90

5.3 Elements . . . . . . . . . . . . . . . . . . . . . 92

5.3 .1 Element Shape . . . . . . . . . . . . . . . . . . . 92

5.3 .2 Element Order . . . . . . . . . . . . . . . . 92

5.3 .3 Integration Method . . . . . . . . . . . . . . . . 93

5.3 .4 Hybrid Elements . . . . . . . . . . . . . . . . . 93

5.4 ABAQUS Benchmarking . . . . . . . . . . . . . . . 94

5.4 .1 Metal Disk Analysis . . . . . . . . . . . . . . . 94

5.4 .2 Rubber Disk Analysis . . . . . . . . . . . . . . . . . 100

5.4.3 Polyurethane Disk Analysis . . . . . . . . . . . . . 107

6 Diaphragm Model 112

6.1 Results . . . . . . . . . . . . . . . . . . 114

7 Discussion, Recommendations and Conclusions $\quad 122$

7.1 Discussion of Objectives . . . . . . . . . . . . . . . . . . 122

7.2 Recommendations. . . . . . . . . . . . . . . . 124

7.3 Conclusions . . . . . . . . . . . . . . . . . 126

$\begin{array}{lr}\text { References } & 128\end{array}$

$\begin{array}{ll}\text { Appendix } & 134\end{array}$

vii 
A MathCAD Metal Plate Simulation

B Williams Model Spreadsheet

C CAE and Input Files 


\section{Nomenclature}

$l_{s} \quad$ Deformed gauge length

$l_{o} \quad$ Original gauge length

$\left(s_{1}, s_{2}, s_{3}\right)$ Eigenvectors of right Cauchy stretch tensor

$\delta_{A B} \quad$ Kroenecker delta

$\delta v \quad$ Arbitrarily small displacement

$\epsilon_{i j} \quad$ Cauchy strain tensor

$\lambda_{1}, \lambda_{2}, \lambda_{3}$ Stretch ratios in 3 principal directions

$\lambda_{u} \quad$ Stretch in the loading direction

C Mooney-Rivlin coefficient for Neo-Hookean form

$\omega \quad$ Eigenvector tensor

$\rho_{o} \quad$ Density of continuum at time $t=0$

$\sigma_{i j} \quad$ Cauchy stress tensor

$\theta \quad$ Angle subtended by deformed gauge length on inflated test specimen

$b \quad$ Body forces (per unit mass) 
$C_{01} \quad$ Second Mooney-Rivlin coefficient

$C_{10} \quad$ First Mooney-Rivlin coefficient

$C_{A B} \quad$ Right Cauchy deformation tensor

$E_{A B} \quad$ Green-Lagrangian deformation tensor

$F_{i, A}, \mathbf{F}$ Deformation gradient tensor

$l_{o} \quad$ Original gauge length on test specimen

$l_{s} \quad$ Deformed gauge length on test specimen

$P \quad$ Hydrostatic pressure

$R \quad$ Orthogonal rotation tensor

$r \quad$ Radius of curvature in an inflated membrane

$S \quad$ Portion of surface subjected to boundary conditions

$s \quad$ External surface tractions or stress vectors

$s_{A j} \quad 1^{s t}$ Piola-Kirchoff stress tensor

$S_{x} \quad$ Portion of surface not subjected to boundary conditions

$t \quad$ Internal stress vector

$T_{A B} \quad 2^{\text {nd }}$ Piola-Kirchoff stress tensor

$t_{o} \quad$ Initial thickness in a test sample

$t_{s} \quad$ Thickness in deformed sample

$\operatorname{tr}(\mathbf{C})$ Sum of the diagonal terms of the right Cauchy stretch tensor, $C_{m m}$ 
$U \quad$ Symmetric positive definite right stretch tensor

$v \quad$ Velocity field

$V_{o} \quad$ Volume of continuum at time $t=0$

W. Strain energy per unit volume

$X_{i} \quad$ Original location of nodes

$x_{i} \quad$ Deformed location of nodes

$I_{A} \quad$ Basis vector in undeformed state

$i_{i} \quad$ Basis vector in deformed state

$I_{i} \quad$ Principal Invariants of right Cauchy stretch tensor 


\section{List of Figures}

2.1 HSVAD configuration and anatomical placement $[2] \ldots \ldots . . \ldots 10$

2.2 Schematic of typical VAD setup showing diaphragm mounted in VAD hous-

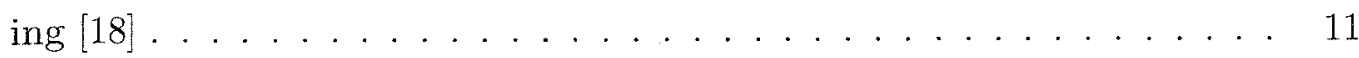

2.3 Novacor pusher-plate design $[19] \ldots \ldots \ldots \ldots 11$

2.4 Diaphragm at various stages of inversion and inflation $[18] \ldots \ldots 12$

2.5 Pressure profile across LVAD diaphragm . . . . . . . . . . . 15

2.6 Air pressure profile behind LVAD diaphragm . . . . . . . . . . 16

2.7 Comparison between the membrane and bending stress within a blood pump diaphragm $[21] \ldots \ldots \ldots \ldots \ldots \ldots$

2.8 Photograph of the isochromatic fringe pattern of polyurethane diaphragm, Chronoflex AR [25] . . . . . . . . . . . . . . . . . . 21

2.9 McMillin Fatigue Test Apparatus $[27] \ldots \ldots \ldots \ldots$

2.10 Close-up of Fatigue Test Apparatus with carbon black rubber mounted . . 25

2.11 Strain at $50 \%$ failure for various candidate VAD biomaterials for cut speci-

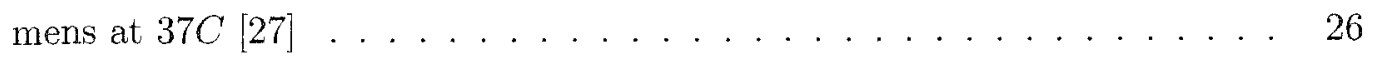

2.12 Strain at $50 \%$ failure for Biospan and Chronoflex AR, cut specimens at

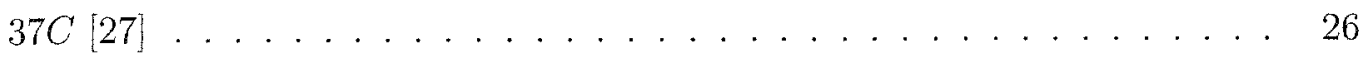

2.13 Cutaway assembly drawing of the Utah-100 total artificial heart with 4 layer Biomer system $[9] \ldots \ldots \ldots \ldots \ldots . \ldots \ldots . \ldots \ldots$ 
2.14 Diaphragm designs in Jarvik and Utah-100 artificial hearts showing inverted position in dashed lines $[9] \ldots \ldots \ldots . \ldots . \ldots . \ldots 27$

3.1 Repeating mer units of ethylene form polyethylene . . . . . . . . . . . . 31

3.2 Entanglement of chains shown on left. The right image shows the motion restricted due to covalent bonds $[35] \ldots \ldots \ldots . \ldots \ldots$

3.3 Tear strength as a function of crosslink density . . . . . . . . . . 33

3.4 Molecular architecture of Biomer . . . . . . . . . . . . . . . . 34

3.5 Segmented polyurethane elastomer: hard and soft segments . . . . . . . 35

4.1 Principal extension ratios in simple extension [38] . . . . . . . . . . . 54

4.2 Principal extension ratios in equibiaxial extension $[38] \ldots \ldots \ldots \ldots$

4.3 Stress-strain response of carbon black rubber in three deformation states[43] 58

4.4 Uniaxial and biaxial folding modes $[21] \ldots \ldots \ldots \ldots$

4.5 Effect of hysteresis on virgin stress-strain curve for Material \#2[39] . . . 59

4.6 Residual strain Material \#2 formulation at varying strains[39] . . . . . 60

4.7 Full curve of uniaxial tensile data for Material \#2 (Biomer clone) . . . . . 62

4.8 Uniaxial tensile data for Material \#1 and Material \#2 (Biomer clones) . . 63

4.9 Mooney-Rivlin Plot of Uniaxial Tension . . . . . . . . . . . . . 64

4.10 Mooney-Rivlin Plot over range of interest (ROI) . . . . . . . . . . 66

4.11 Evaluation of Mooney-Rivlin coefficients shown in Table 4.1 . . . . . . 70

4.12 AXEL apparatus to determine biaxial constants [45] . . . . . . . 72

4.13 Exploded view of base and clamp [46] . . . . . . . . . . . 74

4.14 Nikon Optical Comparator . . . . . . . . . . . . . . 75

4.15 Equidistant point measurement $[46] \ldots \ldots \ldots$. . . . . . . 76

4.16 Inflation pressure-stretch curve for Material \#1 . . . . . . . . . . 79 
4.17 Variable definition for trigonometric relationship to determine radius of cur-

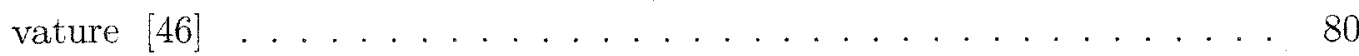

4.18 Biaxial stress-stretch curve for Material \#1 . . . . . . . . . . . . . 82

4.19 Mooney-Rivlin plot for the biaxial strain case for Material \#1 . . . . . . 83

4.20 Evaluation of Mooney-Rivlin coefficients determined from least squares fit over biaxial data for Material \#1 . . . . . . . . . . . . . 85

5.1 Volumetric strain locking in 2D and egg-crate warped mesh [47] . . . . 89

5.2 Load displacement curve for non-linear response . . . . . . . . . . . . . . 91

5.3 Thindisk model used in study with coarse mesh $\leq 150$ elements . . . . . 96

5.4 Boundary conditions for thin disk model. Filled circles indicate no motion in Z-direction . . . . . . . . . . . . . . . . . 97

5.5 Meshed disk with inset showing periphery of disk with built-in, boundary condition along bottom row of nodes . . . . . . . . . . . . 97

5.6 Stress distribution at equally spaced nodes along the pressurized metal disk at $30 \mathrm{KPa} \ldots \ldots \ldots \ldots \ldots \ldots$

5.7 Built-in boundary conditions for axisymmetric model . . . . . . . . . . 101

5.8 Pinned boundary conditions for axisymmetric model . . . . . . . . . . . 102

5.9 Thick rubber disk analysis . . . . . . . . . . . . . . . . . . . . . . 104

5.10 Assumed shape of cap of sphere used in Williams model. . . . . . . . . . 106

5.11 Comparison of response of 1st and 2nd order elements. Rubber is superimposed for comparison . . . . . . . . . . . . . . . . 108

5.12 Material \#1 disk stress analysis for second order, C3D20RH, elements at 30KPa. Rubber analysis is superimposed. . . . . . . . . . . . . . . 109

6.1 Isometric view of diaphragm model . . . . . . . . . . . . . . . . 113

6.2 Evaluation of ABAQUS generated coefficients in biaxial tension . . . . . 115 
6.3 Evaluation of ABAQUS generated coefficients in uniaxial tension . . . . . 116

6.4 Deformation at $0 \mathrm{KPa} \ldots \ldots \ldots \ldots \ldots \ldots$

6.5 Deformation at $7 \mathrm{KPa} \ldots \ldots \ldots \ldots \ldots$

6.6 Deformation at $14 \mathrm{KPa} \ldots \ldots \ldots \ldots \ldots$

6.7 Deformation at $21 \mathrm{KPa} \ldots \ldots \ldots \ldots \ldots \ldots$

6.8 Deformation at $27 \mathrm{KPa} \ldots \ldots \ldots \ldots \ldots \ldots$

6.9 Deformation at $30 \mathrm{KPa} \ldots \ldots \ldots \ldots \ldots$

7.1 Vertical(left) and horizontal(right) orientation of VAD and effect on diaphragm motion $[55] \ldots \ldots \ldots \ldots \ldots \ldots \ldots$ 


\section{List of Tables}

3.1 Hard and soft segments and their chemical formulation . . . . . . . . . 34

4.1 Mooney-Rivlin coefficients for Biomer clones and published Biomer data for strains in uniaxial tension .................... 67

4.2 Precision of instruments for inflation test . . . . . . . . . . . 78

4.3 Mooney-Rivlin coefficients for Material \#1 and published values for Biomer for biaxial tension . . . . . . . . . . . . . . . . . . . . 84

5.1 Deflection at centre for metal disk analysis using various element types and density . . . . . . . . . . . . . . . . . . . . . 98

5.2 Deflection at centre for Hart-Smith and Crisp axisymmetric model . . . . 103

6.1 Strain ranges within which stable response is achieved in ABAQUS for entered raw data. . . . . . . . . . . . . . . . . . . . 114 


\section{Chapter 1}

\section{Introduction}

The target design life for cardiac assist devices is five years [1]. The operation of this device, for this period, subjects the components to approximately 200 million loading cycles which, in turn, makes elastomeric components within them prone to fatigue failure. Membrane abnormalities, such as crack appearance on the surface of the diaphragm, may result in significant mechanical problems cropping up in patients with long-term devices. Indeed, surface cracks which may propagate through flexible polyurethane diaphragm membranes in cardiac assist devices may lead to device failure. This presents a design and manufacturing challenge as reliability in medical devices is critical. This thesis employs a computer and numerical simulation to determine areas of stress concentrations in elastomeric diaphragms and to provide a tool to substantiate any adjustment to the design to relieve the stress, and thus, improve reliability.

\subsection{Background}

Between 1995 and 2000, an estimated 50000 people in Canada developed heart failure each year. In Canada alone, 5000-8000 patients die annually from heart failure [2]. In these patients, modern pharmaceutical treatment fails to improve the cardiovascular blood flow 
and pressure to an acceptable level [3].

Many of these patients can benefit from a transplant. However, in Canada, only 250300 donor hearts become available each year. Due to low donor heart availability; it is estimated that 1500-2000 patients, per year, are left without a chance to receive a natural heart transplant [2]. One option for those suffering from "heart failure", and without a chance to receive a transplant, is to receive assistance from an LVAD, or left ventricular assist device. The LVAD is an implantable mechanical device which receives oxygenated blood from the lungs and pumps the blood via the passive and failing left ventricle to the body and vital organs. One such device is the Novacor LVAD, first introduced in 1984 and currently sold by Worldheart Corporation based in Ottawa, Ontario, Canada. In North America, the LVAD is primarily used as a bridge to transplant(BTT) of a donated heart. Another benefit to LVAD therapy is that the LVAD relieves the cardiac muscle of the work load(to achieve adequate blood flow) and, in some cases, the damaged heart tissue can repair itself and the patient can be weaned off the device [4].

Currently, with the number of patients awaiting a donor heart and in some instances, patients who are unsuitable transplant candidates, the LVAD, and, in particular, the Novacor LVAD, is often relied upon as a long-term implant, greater than 1 year use. [5] [3]. This thesis regards implants $<1$ year as short-term and $>1$ year as long-term as defined by Loisance [5].

The Novacor LVAD, as mentioned earlier, is considered to be one of the most mechanically reliable devices available [2]. Indeed, Worldheart Corporation, the device manufacturer, reports of recipients with more than 4 yrs running time, functioning adequately, with no mechanical failures, and still using their original devices. This, incidentally, is a record in long-term circulatory support. In addition to this, there are clinical studies available that have reported few mechanical failures resulting in premature death from the use of the Novacor LVAD [6] [7]. 
Earlier this year, Worldheart Corporation proposed a next generation of LVAD that offers two significant design improvements on the original Novacor [2]. Firstly, reducing the size of the device is a priority. Current design pulsatile flow LVAD's cannot be used in patients with a small body surface area because of the large pump size [8]. Secondly, making the device fully implantable is advantageous. Infection is a major complication for this type of device as it is implanted subcutaneously in the abdomen and various conduits to the device penetrate the skin.

It is noted from short-term studies and even reports of long-term patients that a particular component, the internal flexible rubber-like diaphragm or sac, is critical to device function. Due to cyclic loading they may also be prone to fatigue failure if stressed in an adverse manner, as will be discussed later. These components not only eject blood from the pump but also act as an interface between the blood and the rest of the mechanical components.

Biomedical grade polyurethane is the material of choice for device manufacturers as they possess good qualities in biocompatibility, flexibility and fatigue. Nevertheless, designing with this material presents a challenge. New diaphragm designs proposed by device manufacturers should be analyzed for stress concentrations and deformation modes. If upstream design concept evaluation is not part of the design cycle, failures may occur as shown by some early studies in the development of cardiac assist devices.

A short-term study released in 1989 [9], on the Jarvik series artificial heart implanted in calves and sheep from $1976-1983$, showed that $30.7 \%$ of its failures were due to mechanical causes. No less than $30 \%$ of these problems were diaphragm related. The diaphragm failures occurred within 268 days of implant. Changes to this diaphragm design alleviated the stress and reduced the number of failures.

A long-term study [3], of 20 Heartmate LVAD's produced by Thermocardio Systems Inc., from 1996-1998, showed one recipient having a technical failure due to diaphragm 
failure after 14 months. The internal diaphragm was found to have developed minor cracks leading to a leakage of blood into the motor chamber causing the device to stop operating. It is important to note that the average support time in this study was $174.6 \pm 175$ days. This particular device was under long-term, $>1 \mathrm{yr}$, use when the device failed. Fortunately, the patient, originally under out-of-hospital care, had the pump exchanged. At the time of the study, the patient returned home to await a natural heart transplant.

In a more recent article in the August 2003 issue of Mechanical Engineering a device failure was reported in the new AbioCor Implantable Heart, manufactured by Abiomed. A long term recipient, surviving close to 17 months, died due to fatigue of the internal membrane [10].

To summarize, there are a number of reasons that motivate this research. The Novacor LVAD, for example, is no longer seen as a bridge to transplant (BTT), but more as long-term support or even permanent support for a failing heart [7]. As implant times increase mechanical failures may emerge as a new problem in long term use [11]. Alleviating stress concentrations may improve confidence in device reliability and possibly lengthen the recommended time for device replacement. Secondly, new and better LVAD and artificial heart designs are anticipated. These devices are expected to be smaller and fully implantable thus requiring component redesign.

The aim of this research is to investigate the potential use of computer simulation, developed from a mechanical-structural perspective, that will assist designers in the investigation of stress concentrations in proposed diaphragm shapes. These locations of stress concentration are possible sources of microcracks and subsequent crack propagation that will eventually lead to device failure [12]. The method used to analyze this problem is the finite element technique. The software package used is ABAQUS/Standard. The inversion of the rubber diaphragm involves large deformations and hence, a non-linear analysis is required. Furthermore, the material itself possesses a non-linear elastomeric stress-strain 
response. ABAQUS can handle non-linear simulations and has an extensive list of nonlinear material models. Many other FEA programs exist, however, given the ease of use and post-processing capability, ABAQUS is the program of choice.

In addition to stress analysis, deformation analysis can be conducted to assist designers in predicting tolerance and fit issues. For example, predicting how the deformed shape of a proposed design appears may affect the housing design or the sizing of other components.

The results of this study may also have potential benefits to the industry. The engineering product development cycles in the biomedical industry often involves prototyping a design, testing and rebuilding [13]. Indeed, the design process consists of trial-and-error iterations prior to a final design. These iterations are costly, time consuming and tax resources [14]. It is established that predictive analysis, through computer simulation, can significantly impact the final design as well as accelerate product development cycles [14].

Finally, the regulatory approval of new designs is a lengthy process in the biomedical industry [14][15]. Computer simulation can be a potential qualification tool to substantiate changes for regulatory purposes. Thus, a tool for designers to substantiate adjustments to the design could not only significantly improve performance and reliability, but also time to market.

\subsection{Thesis Objectives}

The first objective of this thesis is to use the finite element technique to analyze deformation and stress in a typical artificial heart diaphragm shape made from biomedical grade polyurethane material.

Develop a complete set of stress-strain curves for biomedical grade polyurethane necessary to determine the hyperelastic constants for the chosen strain energy model used in the FEA software. 
Verify the use of the 2-term Mooney-Rivlin strain energy density model with biomedical grade polyurethane.

Evaluate the chosen FEA software, ABAQUS for its ability to handle non-linear finite deformation elasticity problems and producing the coefficients for the Mooney-Rivlin model.

State the underlying principles of the ABAQUS software simulation representing the non-linear large deformations of the diaphragm and state the assumptions in boundary conditions and material properties. In doing so, the reader is made aware of the difference in non-linear finite deformation analysis over the infinitesimal strain theory and also how the strain energy model fits into the mathematical formulation.

Identify sources of material data entry error, human error and factors unaccounted for in the model.

Recommend further modelling requirements for true representation, model verification and improvements to material characterization.

\subsection{Thesis Overview}

Chapter 1 serves to introduce the current problem of long term use of LVAD's, to highlight some of the more recent cases of diaphragm abnormalities and to inform the reader that newly proposed designs are smaller. These are motivating factors for the development of computer based simulation to substantiate adjustments to designs without sacrificing device reliability and performance.

Chapter 2 reviews recent literature which describe modelling and analysis of the diaphragm of an artificial heart through computer simulation. Mechanical and environmental stresses are described. Physical material tests pertaining to the long term durability of the diaphragm are mentioned. General LVAD function is reviewed followed by a breakdown 
of the diaphragm problem from a mechanical perspective. The deformation modes of the diaphragm are reviewed.

Chapter 3 is a brief overview of the molecular structure of polyurethane that provides an understanding of the potential impact of any chemical adjustments to the mechanicalstructural model and the stress-strain relationship based on statistical and thermodynamic assumptions of molecular chain theory. This model is a fortuitous and necessary complement to the hyperelastic mechanical model, in uniaxial strain, derived independently from linear elasticity theory detailed in Chapter 4.

Chapter 4 provides a brief introduction to the continuum mechanics theory for the development of the variational function discretized in the finite element model. The polyurethane elastomer is treated as an isotropic, incompressible hyperelastic material. These terms are described. The stress in the material is determined and is simplified for a static deformations analysis. Deformation and strain theory, conservation principles in continuum theory, the principle of virtual work, Dirichlet and Neumann boundary conditions, are used to develop the variational function for the FEA formulation and are all reviewed. The chosen hyperelastic model to represent the strain energy density of the polyurethane biomaterial is the Mooney-Rivlin form.

Two polyurethane materials were used in this study. Both are clones of the original Biomer formulation and are called Material \#1 and Material \#2. A complete set of data for Material \#1 was obtained, through the course of this research. This material is used in the final diaphragm model. Only uniaxial data was available for Material \#2, however this information was helpful in confirming the use of the Mooney-Rivlin model with biomedical grade polyurethane material.

The coefficients of this Mooney-Rivlin form are determined from stress-strain data in uniaxial and biaxial and planar (pure shear tests). The methods to determine the coefficients are described in detail. The biaxial stress-strain data are collected using an 
inflation test. The apparatus and procedures for collecting and processing the data to determine the material coefficients is described. The biaxial and uniaxial material tests and methods to determine coefficients for the chosen hyperelastic model is described in detail. Exclusion of pure shear data is explained.

In Chapter 5 the finite element formulation is discussed with special consideration of the incompressibility constraint on the stiffness matrix and element selection. A Lagrange multiplier constraint is applied to the variational function via the constitutive equation. Numerical methods, such as Newton's method for convergence and the Modified Riks method, resolving the dynamic snap through event is described. To assess the quality of fit of ABAQUS, the pressurization of a metal plate was compared against a hand calculation. The inflation of a rubber disk is compared against physical tests by Oden [16] and finite element results from Hart-Smith and Crisp [17].

Chapter 6 provides the final model of the diaphragm with pinned and built-in boundary conditions of a circular VAD membrane. The model is used with pressures observed behind VAD membranes from fluid (gas or liquid) driven diaphragms.

Several conclusions are presented in Chapter 7, with recommendations for future refinement of the finite element model, for material characterization, and other postprocessing information requests that can be derived from the results. 


\section{Chapter 2}

\section{Literature Review}

Heart failure patients typically have problems with the left-ventricular muscle and thus the LVAD is a therapy of choice rather than opting for a complete artificial heart. This study considers LVAD diaphragms but given the similarity to other cardiac assist devices, the results of this study are not limited in their application. Furthermore, there are many cardiac assist devices for therapeutic use, including the Heartmate, produced by Thoratec, the Novacor LVAD by Worldheart and the AbioCor by Abiomed, to which the results of this study may apply. Furthermore, as mentioned earlier, a new design proposed by Worldheart corporation the HeartSaver Ventricular Assist Device, may also benefit from this research.

The following literature review discusses the working conditions of the diaphragm, studies on stress concentrations and fatigue, understanding the inversion of the diaphragm from a mechanical perspective and early efforts in diaphragm design.

\subsection{LVAD Arrangement and Function}

The configuration and anatomical placement of the next generation HeartSaver is shown in Figure 2.1. The inlet cannula leading to the device from the failed left ven- 


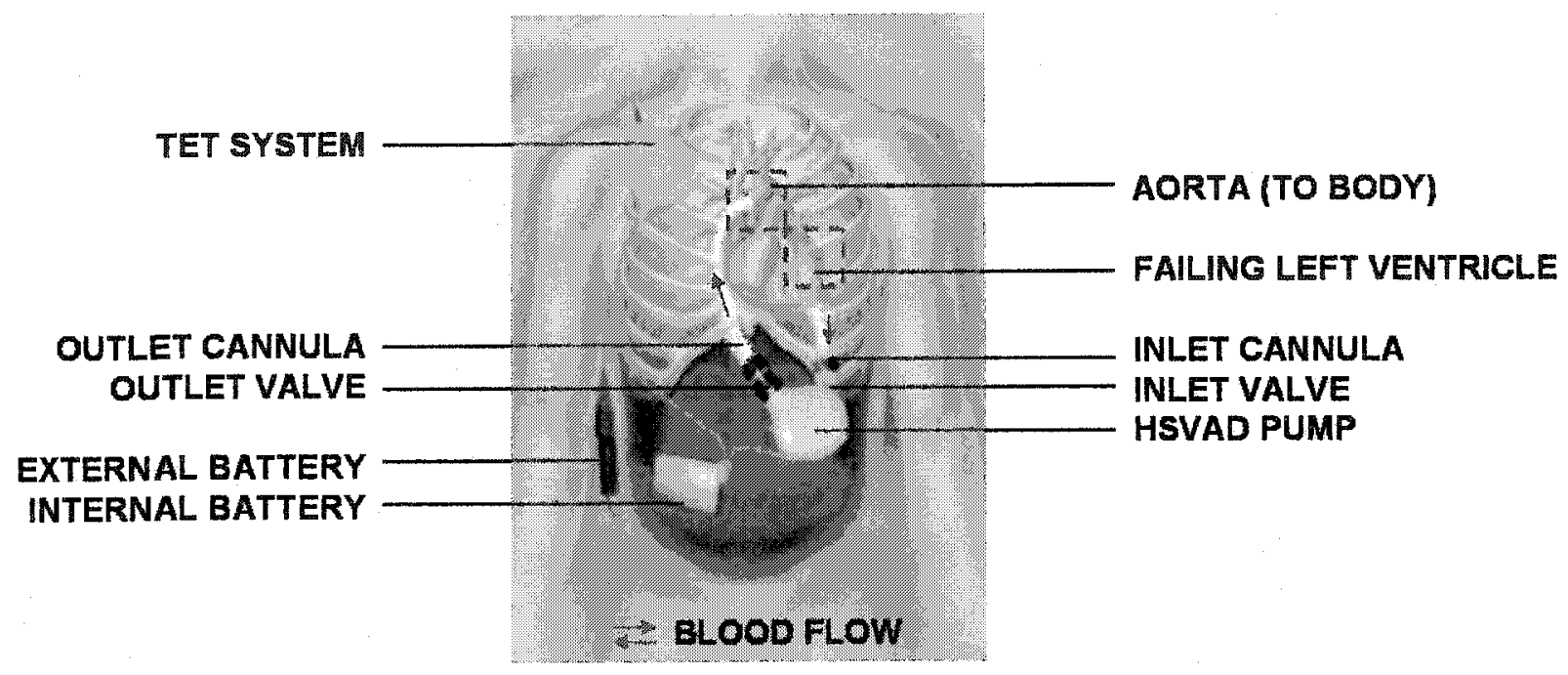

Figure 2.1: HSVAD configuration and anatomical placement [2]

tricle contains a one-way valve. The outlet cannula, with another one way valve, leads from the device to the aorta, a large artery that carries blood away from the heart.

Figure 2.2 shows a typical VAD setup with a diaphragm mounted in the housing. The schematic shows the location of the diaphragm in the housing and the rough dimensions of the radius and the thickness of the diaphragm. The internal diaphragm is in direct contact with the blood and is responsible for driving blood out of the device. The diaphragm is cast into a dome shape and flexes such that it inverts upon itself.

Some LVAD designs actuate the internal diaphragm by means of air flow requiring that a tube penetrate the skin and vent air. With recently proposed, fully implantable devices, such as the new Heartsaver device, it is expected that skin penetration will be removed from the design thus eliminating a source of infection in the patient. Fully implantable LVAD designs include diaphragms that are driven forward by fluids such as silicone oil or by a pusher-plate as shown schematically in Figure 2.3 .

Power to the device is provided by means of a transcutaneous energy transfer (TET) system that allows energy to pass through the skin by electromagnetic induction thus eliminating the need for a power cable to penetrate the skin. The device is also wireless, 


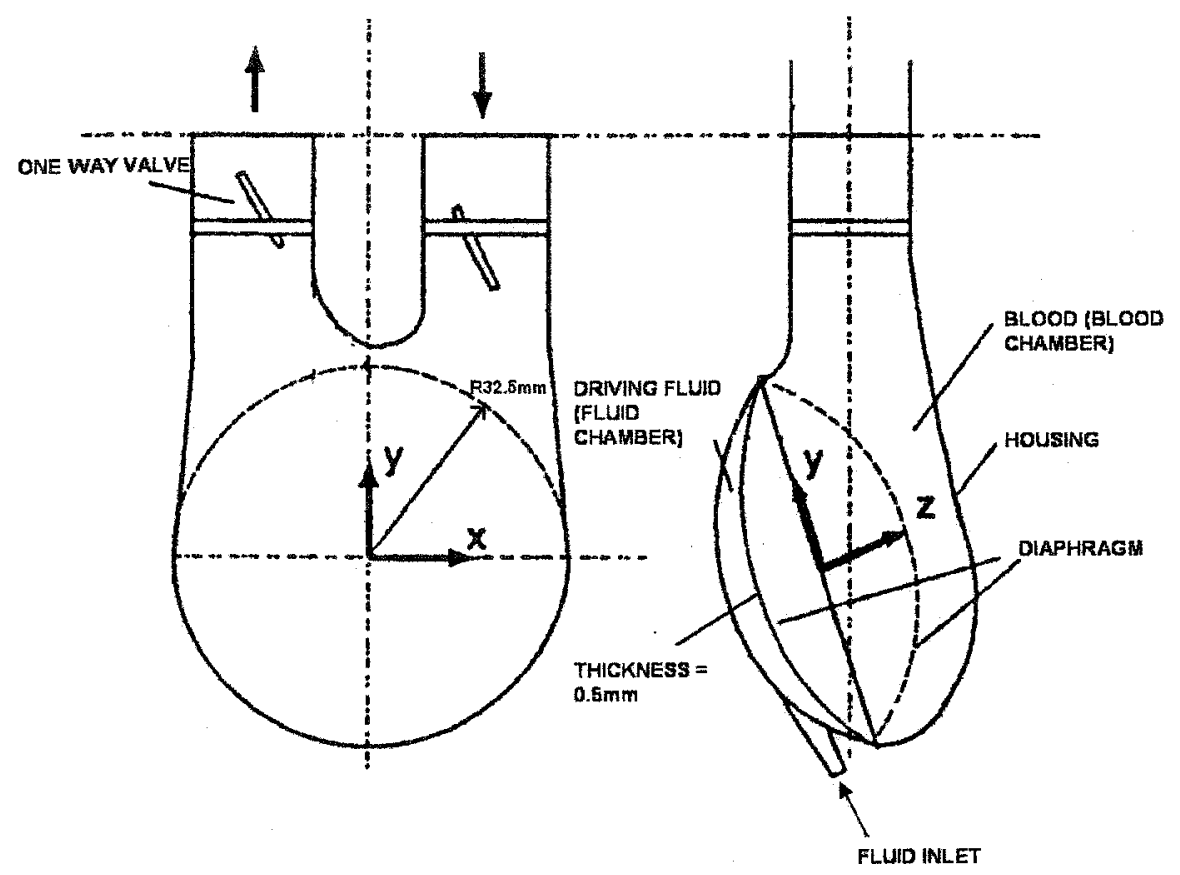

Figure 2.2: Schematic of typical VAD setup showing diaphragm mounted in VAD housing [18]

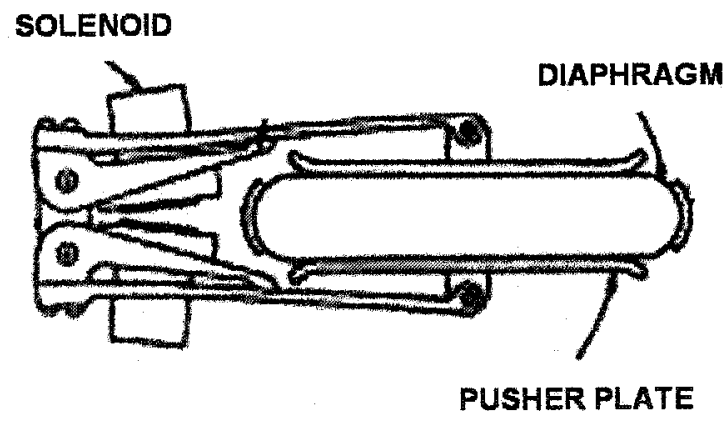

Figure 2.3: Novacor pusher-plate design [19] 


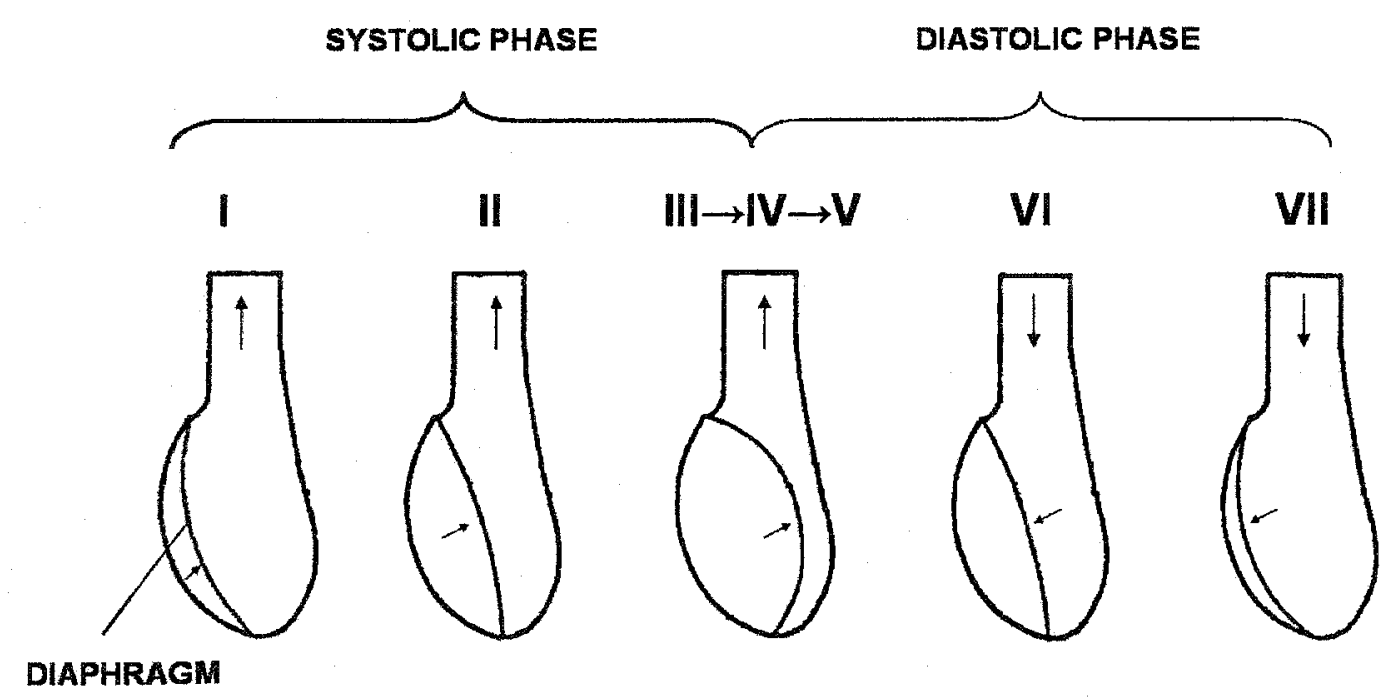

Figure 2.4: Diaphragm at various stages of inversion and inflation [18]

thus parameters, such as the beat rate, can be controlled remotely. External batteries are worn in a holster or the device can be plugged into a wall unit.

Figure 2.4 shows the various locations of the diaphragm at different stages of blood ejection. Much like the human heart, the LVAD creates blood flow through a pumping action as well as imitating the two cardiovascular states of diastole and systole as well as stroke volume. The various stages of inversion of the internal diaphragm corresponds to the beat of the natural heart and as such the states are described in terms of physiological terms. Systole is the state of the cardiovascular system when the heart is ejecting blood, causing systemic blood pressure to increase. Diastole is the state of the system when the heart is at rest and, during this phase, venous pressure causes blood to flow back from the body and into the heart. The stroke volume is the total amount of volume leaving each ventricle during each beat of the heart. The stage numbering refers to the deformed states of the diaphragm and are discussed later in terms of pressure development behind the diaphragm.

The implanted LVAD runs in parallel with the failed heart. During the diastole phase, 
blood flows through the failed left ventricle and into the inlet cannula towards the LVAD. Pressure in the veins leading to the heart causes this filling. The diaphragm is forced back to allow blood to fill the chamber as shown in stages 6 and 7 in Figure 2.4. After detecting that the LVAD is full, a feedback controller initiates a sequence of events to create positive pressure behind the diaphragm driving it and the captured blood forward. The pressure created by the ejecting blood immediately shuts the inlet valve, cutting off filling. Simultaneously, the outlet valve is forced open causing the captured blood to pass into the outlet cannulae and directly into the aorta as shown in Figure 2.1.

After being emptied, the outlet valve closes from back pressure in the outlet cannulae. Once again, central venous pressure pushes the inlet valve open and the diaphragm back allowing blood to fill the chamber.

\subsection{Diaphragm Requirements}

The average resting heart rate for an adult human being is approximately $60-75$ beats per minute. This suggests the heart experiences over 40 million beats per year. The natural heart creates flow rates up to 25 litres per minute, and creates pressure up to $150 \mathrm{mmHg}$. This pressure is required to drive the blood to the extremities of the cardiovascular system. The expected flow rate with normal blood pressure and resting heart rates is typically $6 \mathrm{~L} / \mathrm{min}$. The flow is delivered in a pulsatile manner, reproducing the heart's natural rhythmic beating. Although, there are other devices that produce non-pulsatile blood flow, it is shown that pulsatility is a necessary requirement for optimum organ function [20]. Given these requirements, the natural heart produces up to 5 watts of fluid power and operates over the lifespan of a human at approximately 80 years.

As mentioned earlier, the diaphragm is a particularly critical component of the device. It not only drives the fluid forward but acts as the barrier between the body and the rest 
of the mechanical components of the device. To develop adequate pressure and flow rate in the body, the diaphragm is expected to work under strenuous cyclic loading conditions. The selected diaphragm material and shape must withstand continuous cyclic loading, up to 200 million cycles over 5 years, the expected life span for these devices. As pressure develops behind the diaphragm, it must not rupture when fully inverted. It must also be flexible enough to easily invert to its new position. The following sections detail the investigations into the motion and deformations of the diaphragm as well as fatigue studies.

\subsection{Pressure Development Behind the SAC and De- formation}

As a first approach, this thesis considers induced motion and deformation from fluid pressure, pneumatic or hydraulic, versus contact pusher-plate driven diaphragms ${ }^{1}$.

Data collected on the pressure development behind the sac provides useful information regarding the way in which the diaphragm deforms. Jin and Clark [18] produced a pressure development profile as shown in Figure 2.5. Data were collected by means of a pressure transducer tapped in the outlet cannulae and another located in the housing around fluid chamber and behind the diaphragm as shown in Figure 2.2. The absolute values from this study are not applicable to our design owing to differences in material stiffness, dimension and driving fluid. Nevertheless, a review of the pressure values from the study offers much insight into the problem.

The study revealed that a maximum air pressure of $240 \mathrm{mmHg}$ was required to push this particular diaphragm forward. This fluid pressure is required to overcome the stiffness and inertia of the diaphragm, the flow inertia and flow friction. The pressure readings were

\footnotetext{
${ }^{1}$ To model the metal/rubber contact requires the use of contact elements, a feature also available in ABAQUS
} 


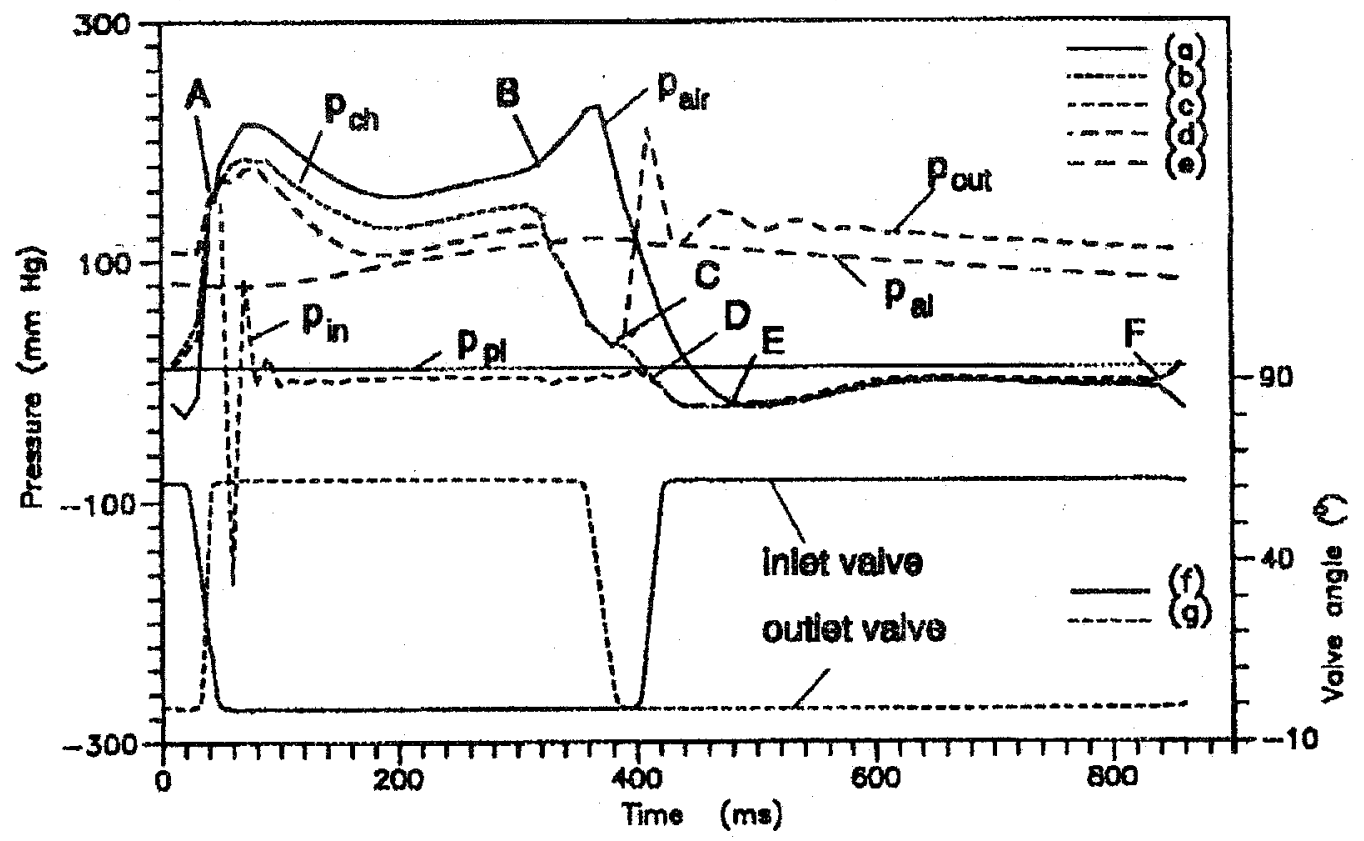

Figure 2.5: Pressure profile across LVAD diaphragm

taken to maintain a flowrate of $4 \mathrm{~L} / \mathrm{min}$ and a beat rate of $70 \mathrm{bpm}$ [18]. The stroke volume is the amount of fluid evacuated by the diaphragm during pulsation. This was observed to be $60 \mathrm{~mL}$.

Jin and Clark used a simpler model to investigate the fluid pressure development behind the diaphragm at various states in diaphragm deformation. They identified 8 stages in diaphragm deformation as shown in Figure 2.6. The stages of each state of the inverting diaphragm is described below. For a physical representation of the stages refer to Figure 2.4 .

\section{Stage 1: Negative Tension Release}

After the captured blood has filled the blood chamber, the diaphragm is stretched towards the base. As fluid flows into the LVAD, pressure is increased behind the sac with relatively low change in volume in the fluid chamber behind the sac. Pressure increases until tension in the stretched diaphragm is released. This can be shown in equations 2.1 


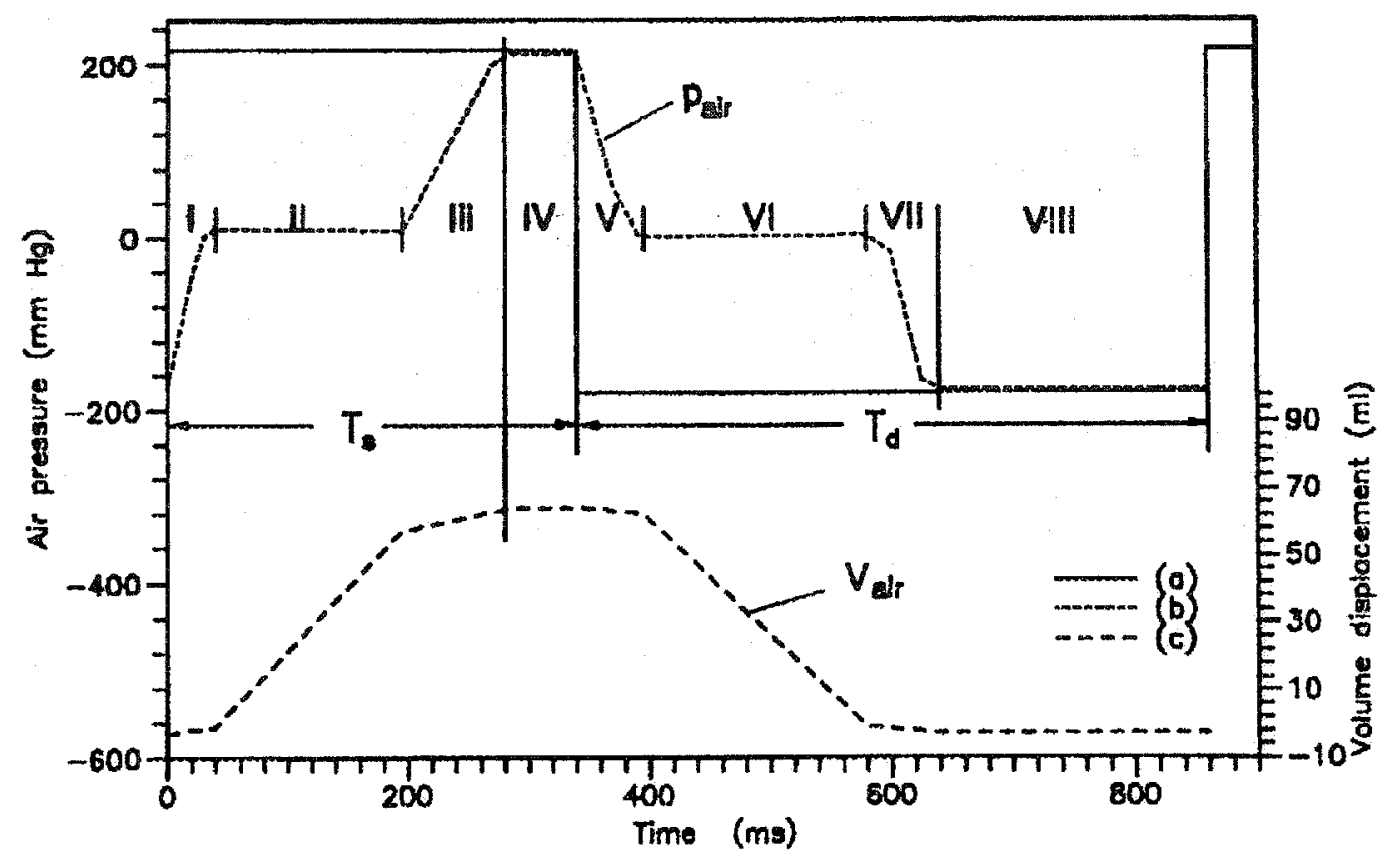

Figure 2.6: Air pressure profile behind LVAD diaphragm

and 2.2 as follows:

$$
\begin{gathered}
\triangle P=P_{\text {fluid }}-P_{h} \\
P_{\text {fluid }}=P_{h}+\triangle P_{o}+\triangle P_{i}+\triangle P_{f}
\end{gathered}
$$

where $\triangle P$ is the space-mean pressure difference, $P_{\text {fluid }}$ is the pressure of the driving fluid, $P_{h}$ is the hydrostatic pressure to overcome, and $\Delta P_{o}, \triangle P_{i}, \triangle P_{f}$ are the pressure differences required to overcome the stiffness and inertia of the diaphragm, the flow inertia, and the flow friction, respectively.

\section{Stage 2: Positive Inversion}

$P_{\text {fluid }}$ is held at a constant pressure and the result is constant volume filling, the fluid chamber fills at a constant rate. The diaphragm bends and buckles during this stage, 
creating folds in the material. At a certain point the diaphragm reaches a limit point in the load-displacement curve and snaps to a new position. The snapping transition is not immediate as it occurs very slowly against a pressure load and the event is dampened by the two fluids on each side. Once the diaphragm is fully inverted this stage ends.

\section{Stage 3: Positive Stretch}

During this stage the diaphragm is fully inverted and now begins to experiences hoop stress. As fluid continues to flow in, a sharp increase in pressure is observed and the rate of change of volume decreases. This stage represents the elasticity of the diaphragm as the diaphragm is now stretching.

\section{Stage 4: Positive Equilibrium}

An equilibrium is reached where the volume in the fluid chamber has reached its maximum.

The rest of the stages, 5 to 8 , continue in a reversed manner beginning with a positive tension release, negative stretch, negative inversion and a negative equilibrium, respectively.

With the data collected shown in Figure 2.6 an estimation of the value of pressure representing the stiffness and inertia of the diaphragm, $\triangle P_{o}$, can be made. Neglecting frictional forces, for the VAD diaphragm shape and composition used in this experiment the average spatial pressure difference to overcome $\Delta P_{o}$ is $4 \mathrm{mmHg}$. This represents a constant stiffness that works against the effective driving force on the blood flow [18].

It is important to note the stretching of the diaphragm in late systole also results in a volume of blood leaving the chamber. By performing a static test on the VAD, Jin and Clark showed that during stage 3 , when the diaphragm is stretching, the volumetric output is almost directly proportional to the applied pressure. Furthermore, $10.5 \%$ of the nominal stroke volume is expelled during stage 3. 


\subsection{Mechanical model}

For the finite element analysis, a breakdown of the pressurization of the fluid chamber and the deformation of the diaphragm from a mechanical perspective is necessary. The process can be described as the conversion of energy from mechanical work to kinetic energy and then finally to shear deformation energy. It is a continuous process and occurs during complete inversion of the diaphragm shown in Figure 2.4. The main assumption is that mechanical work done is stored as shear deformation energy and thus, a static analysis is proposed as a first approach. Volumetric deformation is neglected as the rubber is treated as a nearly incompressible solid.

During stage 2 the diaphragm motion is described mechanically as bending and buckling. This motion produces folds in the material. A study by Zartnack [21] shows that the folding pattern in bending and buckling results in stress concentrations and that those regions are in a state of biaxial deformation and stress. Results showing the stresses in the end-systolic position are not as large as stresses in biaxial folds. The strain in biaxial folds is 5-10 times higher than the strain in uniaxial folds [21]. Bending stresses are higher by approximately a factor of 10. To change this result, Zartnack adjusted diaphragm thickness and dimensions. This folding pattern is shown in the Figure 2.7. The stress and deformation pattern observed during this stage, will eventually favour the creation and appearance of cracks [22].

Following the stages identified by Jin and Clark, during stage 2 the inlet pressure is held constant, as shown in Figure 2.6, and a pressure differential exists in order to create the forward motion of the diaphragm. Thus, the hydraulic or pneumatic pressure behind the diaphragm is considered constant. A $\triangle P$ can be applied to one side of the diaphragm to create the forward motion.

As mentioned, the continuous process is dynamic, however, in a static analysis it is 


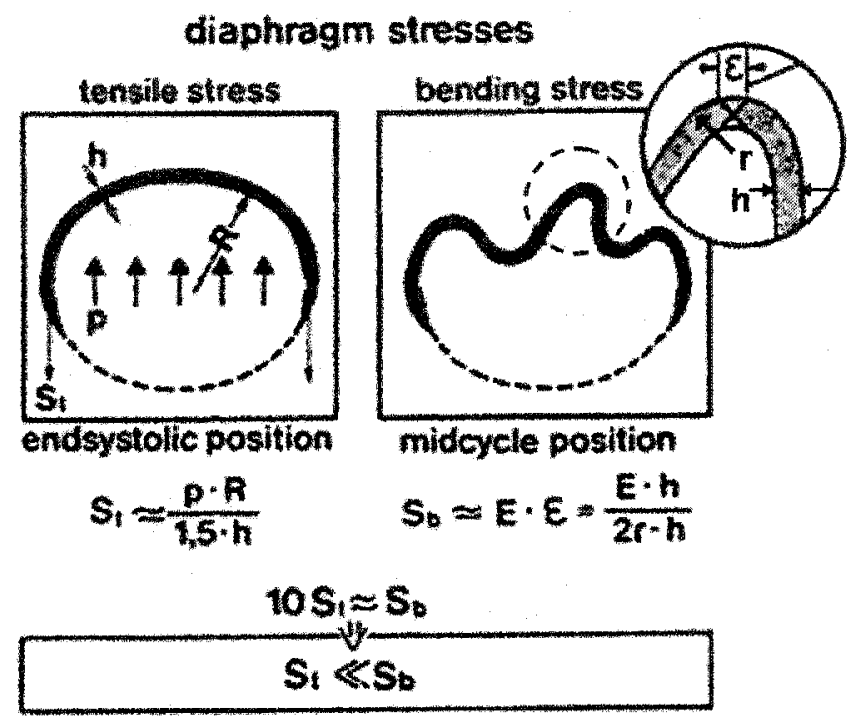

Figure 2.7: Comparison between the membrane and bending stress within a blood pump diaphragm [21]

expected that as an incremental pressure step is induced, the same deformation pattern will be observed at each stage of inversion. And, though, oscillations about each deformed position may occur, a mean deformed shape is preserved.

The surface to which the pressure is applied rotates and stretches, thus there is a stiffness associated with the applied load: a "load" stiffness matrix. Some of the energy is absorbed by the diaphragm and some of the energy is used to create mechanical work. In the finite element implementation, this load-stiffness response is resolved by the use of hybrid elements.

\subsection{Finite Element Analysis of Diaphragms}

Finite element analysis of diaphragms in ventricular assist devices was performed by Knierbien, et al. [23](1988). It was assumed that the diaphragm deforms symmetrically and found that higher material stresses occurred in the central part of the diaphragm and 
in regions where membrane folding occurred, making them more vulnerable to sources of crack appearance. Chatel et al. [24](1997) used a computerized study to design a diaphragm sac. The work used shell elements and examined only membrane stresses and strains. The method was used as a first approach to estimate the feasibility and realism of a concept before producing a prototype. The study showed that stresses at the biaxial folds remained below $3 \mathrm{MPa}$ and strains below $20 \%$. Uniaxial stress-strain curves were generated with their material of choice before and after being cycled and no change in the stress response occurred in the region of the stresses and strains found in their finite element analysis.

\subsection{Photoelastic Analysis}

The appearance of high stress concentrations in diaphragms is an unresolved issue in designs after more than 20 years of development. Even the most recently developed designs can have potentially vulnerable stress concentrations as observed by McColgan [25](2001) by means of a photoelastic investigation on a previous version of the HSVAD diaphragm. Photoelastic stress analysis is accomplished by shining polarized light though a material and subjecting the material to a state of stress. As shown in Figure 2.8 there appears to be a high stress region in the lower right corner of the diaphragm. The resulting fringe pattern that forms on the surface of the diaphragm can be correlated back to a value of stress using the following equation:

$$
\sigma_{1}-\sigma_{2}=\frac{N f_{\sigma}}{h}
$$

where $h$ is the thickness of the material, $f_{\sigma}$ is the material fringe value and is property of the model material for a given wavelength, and $\mathrm{N}$ is the fringe number.

It can also be shown, from shell theory, that bending strains exceed $50 \%$ when the radius 


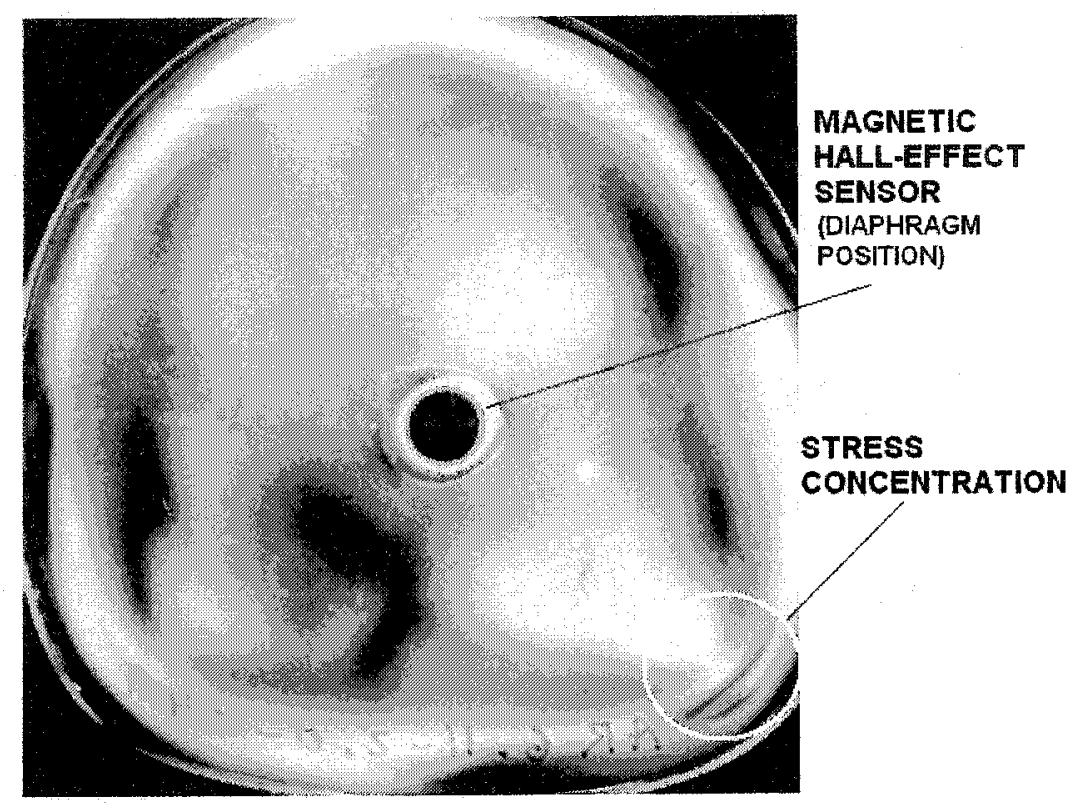

Figure 2.8: Photograph of the isochromatic fringe pattern of polyurethane diaphragm, Chronoflex AR [25]

of curvature approaches the thickness of the diaphragm using the following relation:

$$
\varepsilon_{b}=\frac{t}{2 r_{a v g}}
$$

where $t$ is the thickness of the diaphragm, $r_{a v g}$ is the radius of curvature and $\varepsilon_{b}$ is the bending strain [25] also shown in Figure 2.7.

It is important to note that it is possible to analyze stresses with this method, however, setup of such testing and data collection is time consuming for the analysis of new shape designs and material choices. Thus, the use of numerical methods and non-linear finite element analysis is attractive as, once a model and procedure is developed, evaluating changes and new designs is quicker. 


\subsection{Fatigue Analysis}

Fatigue is a mode of failure which begins with the appearance of stress concentrations followed by the propagation of the crack through the material due to cyclic loading and eventual failure of the component [12]. The phenomenon is immediate and catastrophic.

Specific material composition issues are an important factor affecting the process of fatigue and failure. Material properties and stress development affect each other and changes to the composition can be detrimental. For example, chemists now understand that increasing the proportion of hard segment, explained later in Chapter 3 and Figure 3.5, of the polymer chain may improve biocompatibility [26], but the change also increases the fatigue crack propagation as well as creating a stiffer material [12]. This thesis identifies the material aspect of fatigue as contributing, in parallel, with stress analysis, in the complete picture of fatigue.

Comparative studies of elastomers with different chemical formulation, silicone versus polyurethane, for example, may have relatively minor differences in standard mechanical tests, however, they perform differently when dynamic fatigue and crack propagation resistance is investigated [27] [28] [29].

Many studies evaluating fatigue in candidate biomaterials for ventricular assist devices compare various polyurethane compositions [12] or other material candidates such as silicone [27]. One notable method of inducing fatigue, looks at how long a biomaterial will last if a major flaw were to appear and that flawed region is under uniaxial tension [27]. Other studies looked at the effect of blood, or driving fluids such as warm dry air or silicone oil on material behaviour

Fatigue in biocompatible polymers has been investigated by many researchers. These studies were conducted during the mid-1980's and detailed test methods to rank potential biomaterials used in Ventricular Assist Devices using accelerated fatigue conditions. These 
accelerated studies provide the means to obtain a ranking of materials based on similar environmental conditions but do not provide definitive results on the cycles to failure. Many of the studies use Biomer as a standard material ${ }^{2}$. The key researchers and studies summarized below.

Hayashi The investigations by Hayashi et al. showed that mechanical properties may alter after implantation and after cyclic loading is induced [30] [31]. Their studies showed that after Biomer samples are cyclically stressed, the tensile strength and ultimate elongation at break reduce while the elastic modulus increases slightly. These changes are said to occur due to contact with blood rather than mechanical fatigue. For example, a study by Orang et al. [32], showed that aged Biomer samples removed from saline solution, without drying, showed a $40 \%$ increase in ultimate strain, and thus simply immersing the Biomer material in saline can significantly affect its mechanical properties even without cycling.

Kardos Kardos et al. [33] showed that no long terms tests are required for fatigue estimates if information on crack propagation and standard material stretch data is obtained in the uniaxial and biaxial states. A relation is presented that can provide cycles to failure at a given stress level. Cycles to failure are published for uniaxial states for Biomer [33], but the more representative biaxial state data is not published although a novel method for determining biaxial stretch is given [34].

McMillin McMillin showed that fatigue testing of standard tensile dogbone shaped specimens, with a $1 \mathrm{~mm}$ cut, in blood and saline environments can differentiate materials based on their fatigue qualities. A picture of one mounted specimen is shown in the schematic Figure 2.9 and the picture shown in Figure 2.10. One end of the sample is mounted to a

\footnotetext{
${ }^{2}$ Since Biomer was removed from the market due to liability issues, artificial heart and LVAD companies have been searching for a replacement. Presently, the most common replacements are clones of the Biomer molecular formulation [15].
} 
stationary ring connected to the frame and the other end is connected to rods that oscillate vertically and create a cyclic motion at a desired frequency.

The study can be viewed as how long a diaphragm can last if a major flaw at the surface of the diaphragm were to appear and the applied stress is uniaxial in the region of the stress concentration. Biomer was shown to have better times to failure in blood and saline environments, compared with other potential candidate materials. Using such methods, a selection of a particular chemical formulation material, can be made on the basis of fatigue. A ranking of cycled biomaterials, with a $1 \mathrm{~mm}$ cut, in various environments, is shown in Figure 2.11.

From this figure it can be deduced that for Biomer, cycled in blood, if uniaxial stresses were to be induced on a major flaw, $1 \mathrm{~mm}$ cut, there is a $50 \%$ probability the diaphragm would fail if $23 \%$ strain is observed in uniaxial tension.

A more recent investigation by Doshi [29], used the same test methods and equipment as developed by McMillin. The study looked at Biospan $\mathrm{C}$ and Chronoflex AR, two potential candidate biomaterials. Biospan $\mathrm{C}$ is produced by the Polymertech Group and Chronoflex AR is produced by Cardiotech. Both are similar in composition to Biomer. Results from cycling in dry air environment were similar to Biomer. The time to failure reduced when samples were immersed in decamethyltetrasiloxane, silicone oil, a potential driving fluid for an old HeartSaverVAD version as shown in Figure 2.12

\subsection{Early Efforts in Diaphragm Design}

The discussion of deformation modes and the review of photoelastic and fatigue studies provides perspective on the nature of diaphragm abnormalities such as crack appearance. Insufficient analysis or inadequate design of the diaphragm may result in crack appearance even with the most advanced elastomeric materials available, such as biomedical grade 


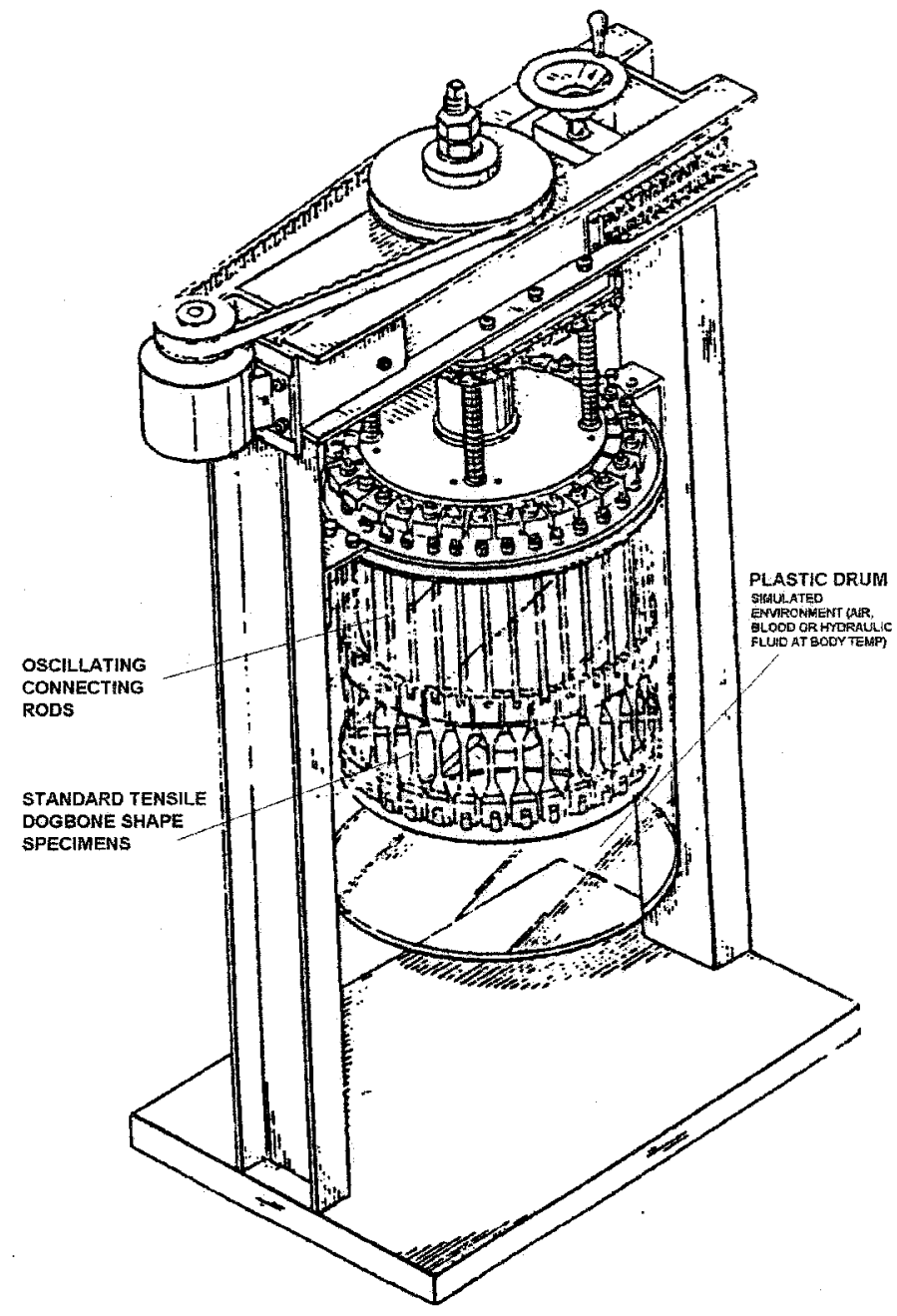

Figure 2.9: McMillin Fatigue Test Apparatus [27]

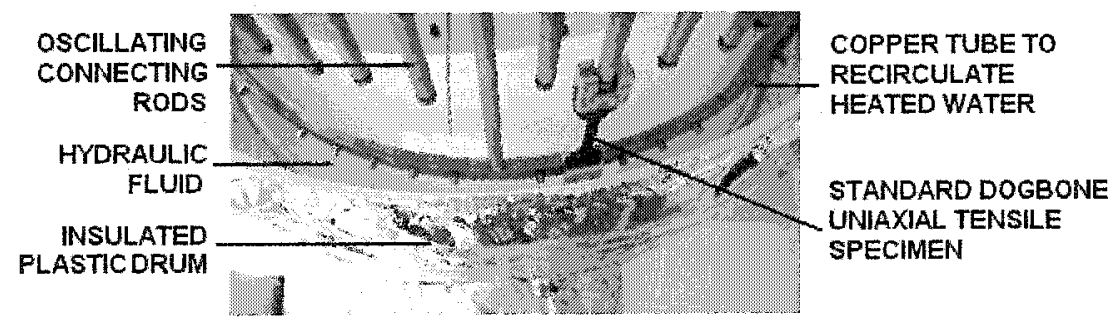

Figure 2.10: Close-up of Fatigue Test Apparatus with carbon black rubber mounted 


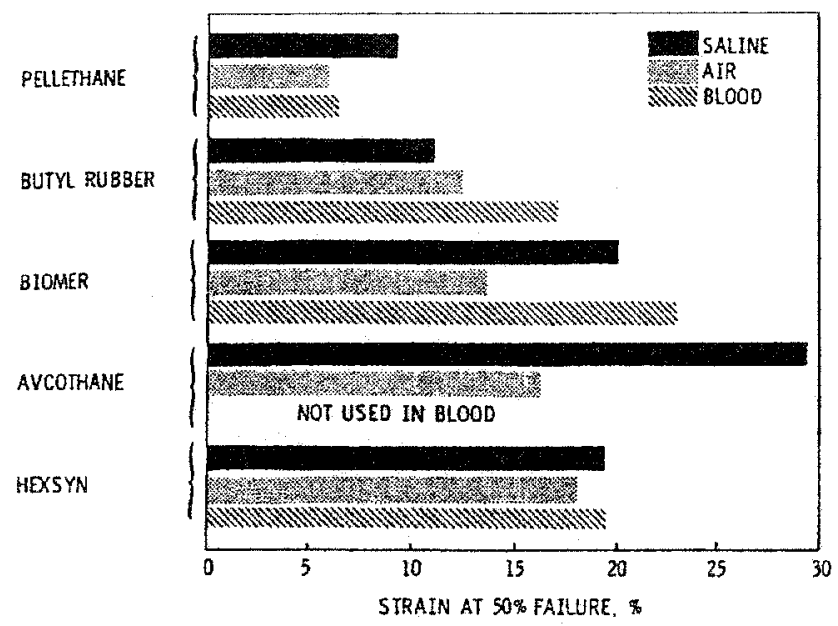

Figure 2.11: Strain at 50\% failure for various candidate VAD biomaterials for cut specimens at $37 C[27]$

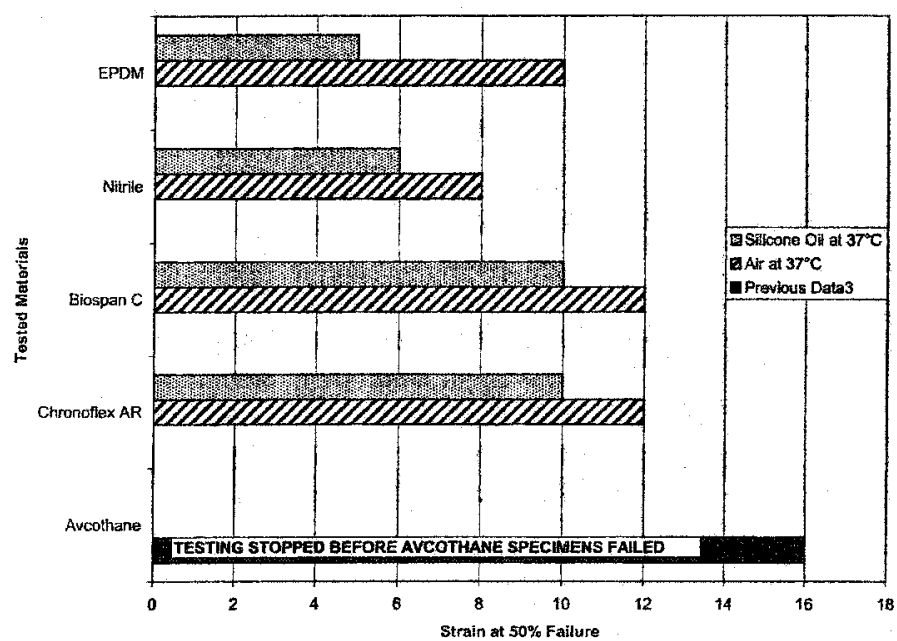

Figure 2.12: Strain at 50\% failure for Biospan and Chronoflex AR, cut specimens at $37 C[27]$ 


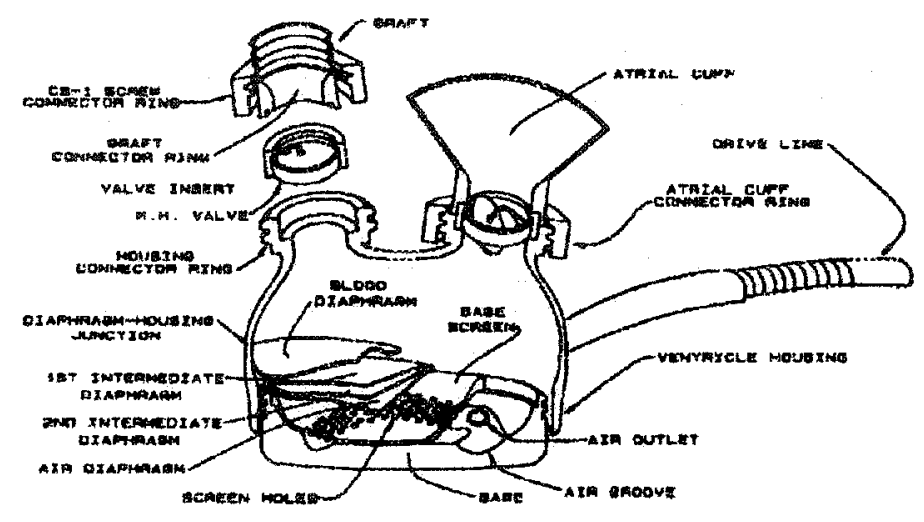

Figure 2.13: Cutaway assembly drawing of the Utah-100 total artificial heart with 4 layer Biomer system [9]
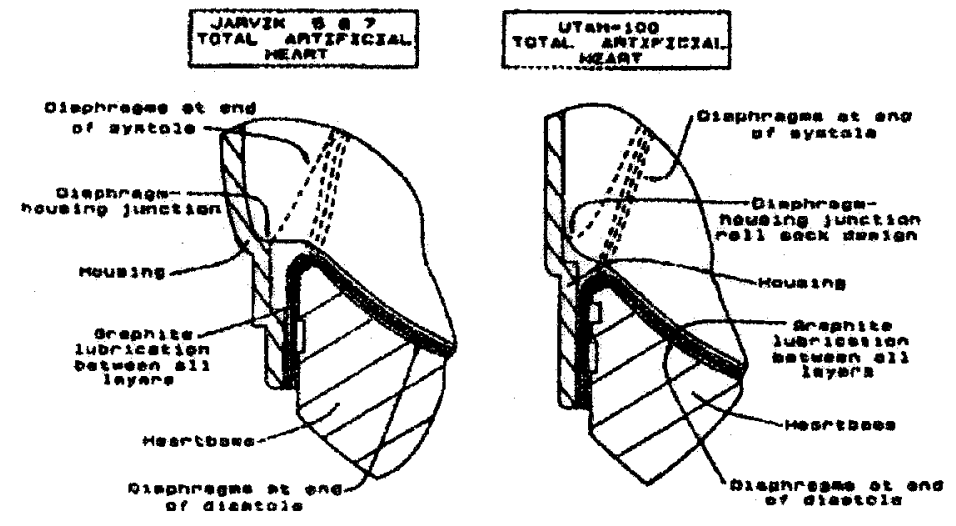

Figure 2.14: Diaphragm designs in Jarvik and Utah-100 artificial hearts showing inverted position in dashed lines [9]

polyurethane.

The aforementioned 1976-1983 study on the Jarvik Total Artificial Heart and Utah Total Artificial Heart designs [9], by Dew et al exemplifies the need for proper analysis. The diaphragm used in both artificial hearts was made from Biomer and both used a 4 layer Biomer system as shown in Figure 2.13.

Failures of the Jarvik Total Artificial Heart were characterized by creases and pinhole leaks in the blood diaphragm, tears at the diaphragm-house junction and bubble defects. A total of 11 of 114 implants were affected by these issues with a crease or hole in the 
blood diaphragm occurring in 5 of those failures. Two of the failures were catastrophic.

The major difference between the two is the diaphragm housing junction (D-H) junction as shown in Figure 2.14, the point of greatest flexing. A "roll sock" design, was implemented in the Utah-100 TAH's to relieve stresses. Of the 27 Utah-100 TAH's, with the "roll sock" design, no creases or tears were observed. It is important to note that the mean duration of implantation was $72.9 \pm 82.1$ days and for the Jarvik series the mean duration was $49.3 \pm 61.3$ days indicating that these were both short term studies.

This study exemplifies the importance of upstream design concept evaluation as stress analysis is a significant factor in the definition of component function. This thesis proposes that the a major causative factor in failure are stress concentrations. The locations of stress are the initiators for microcracks leading to crack propagation in the material and ultimate failure of the diaphragm [22].

The failure of such devices can be approached from a mechanical-structural point of view where we consider the motion of the diaphragm itself as a contributing factor in device failure. This approach was taken by Ionita [22] in research regarding diaphragm accumulators:

"The main cause of the creation of cracks, and untimely failure of the rubber diaphragm is a mechanical cause, due to the deformation of the diaphragm."

Inverted positions of the diaphragm evolves through the problem of buckling, post buckling (dynamic snap through event) and the load response through the evolution. Each step is explained in subsequent chapters after a brief review of the molecular structure of polyurethane. 


\section{Chapter 3}

\section{Molecular Theory of Polyurethane}

Elastomeric materials can be deformed to relatively large extent and elastically spring back to their original form. This makes polyurethane suitable for use as a diaphragm material as it is very flexible. Also, diaphragms are required to have a smooth, low-friction surface to safely eject blood out of the sac. This can be obtained from cast polyurethane parts creating a smooth low friction surface that appears clean and thrombus free upon excision. There are other biomaterials available [26], but many have been eliminated for use in long term application due to degradation, thrombosis or flex life [26].

\subsection{Thermoplastic Polyurethane}

Polyurethanes have many industrial applications and are found to have properties excelling in:

- blood compatibility

- hydrolytic stability

- abrasion resistance

- physical strength

- flexure endurance [26] 
Polyurethane began its use in artificial heart technology with one notable study, by Kolff et al. [26], who were able to implant a blood pump with a polyurethane diaphragm in a dog allowing it to survive for 20 hours.

Currently, for heart valves, the use of thermoplastic polyurethane reduces the requirement for patients to remain long-term on anti-coagulant drugs [28]. There is an abundance of research on polyurethane material for use in biomedical application. The research on polyurethane materials is directed mostly towards the specific blood-surface interaction involving:

- effects of flow

- shear rate

- leukocyte and erythrocyte adhesion

- plasma protein adsorption

- platelet deposition [26]

The present market of biomedical grade polyurethanes is based on numerous attempts to adjust molecular chemistry and fabrication techniques to optimize the aforementioned properties. Two approaches are taken to optimize the surface contacting the blood: (1) form a blood lining called a pseudointima (PNI) or (2) minimize cellular or protein reactions at the surface by making a very smooth surface.

Good smooth surfaces can be developed with solvent cast manufacturing process and/or by using thermoplastic polyurethane. A smooth surface will appear clean and thrombus free upon inspection after excision [26].

\subsection{Elastomers Background}

The rubber-like phenomena of elastomeric polymers can be explained by the microstructure of the material. All polymers are composed of molecular chains of repeating 


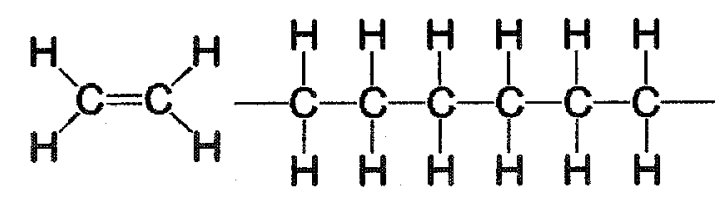

Figure 3.1: Repeating mer units of ethylene form polyethylene

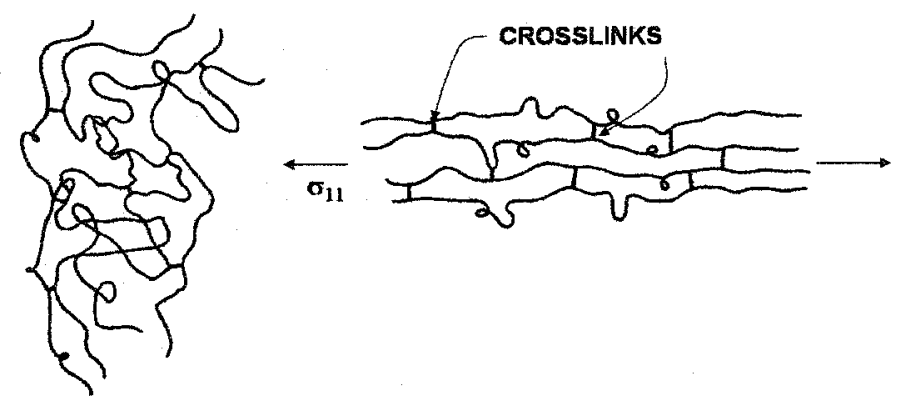

Figure 3.2: Entanglement of chains shown on left. The right image shows the motion restricted due to covalent bonds [35]

monomer units. A mer is the single unit of a polymer. Polymers such as polyethylene, shown in Figure 3.1, contain repeating units of ethylene functional groups.

Polymers exhibit valuable properties and have a variety of applications. Their macrostructure can be thought of as shredded paper, or spaghetti; the more entangled the chains, the more difficult it is to pull them apart. Figure 3.2 shows the entanglement of the polymer chains before and after extension.

Polymer science shows that some types of polymers have molecular chains or polymer backbones that become more ordered by aligning themselves longitudinally, like "uncooked spaghetti". These are called crystalline materials. Conversely, other polymers form a random "cooked spaghetti" structure and are called amorphous polymers [36].

Alignment creates secondary bonds between the chains. These secondary bonds, are much smaller than the primary bonds of the main chain and are affected by the distance between the chains. In fact, the strength of the bonds is equal to $\frac{1}{d^{2}}$. The bonds are composed of hydrogen bonding and van der Waals forces, In crystalline regions the result 
of many secondary bonds due to very closely packed molecules results in an overall very strong bond. Thus, highly crystalline materials have high elastic modulus, making the polymers harder and stiffer.

Thus, in addition to entanglement of the chains, mechanical properties are affected by the following factors and examples:

- intermolecular forces: primary covalent carbon-carbon bonds

- secondary forces between chains: hydrogen bonding, van der Waals forces

- chemical structure: large side groups prevent slippage of chains

Most elastomers such as carbon black filled rubber are composed of molecular chains that are highly coiled giving an amorphous, "cooked spaghetti", macrostructure. As the material is stretched, covalent bonds between the chains act as pinning points from further extension as shown in Figure 3.2. As an example, latex from the sap of tropical trees is heated with sulphur in a process called vulcanization. This creates crosslinks between the long chain polymers and prevents the chains from slipping past each other and from contracting into knots [37].

With carbon-carbon bond rotation the chains will partially unkink and uncoil around each other as they become extended [35]. It is important that elastomers be lightly crosslinked. Unvulcanized rubber, for example, is soft, tacky and is highly susceptible to abrasion. With vulcanization, the modulus of elasticity, and tensile strength increases. The crosslinking is also responsible for the thermoset nature of elastomers, ie. upon heating, the chains don't easily slip past each other, or melt as easily and the covalent bonds must first oxidize. It is important to note that as crosslink density is increased the tear strength increases to a limit, after which there is a marked decrease. The material becomes more brittle and less tough (and less resistant to crack propagation). 


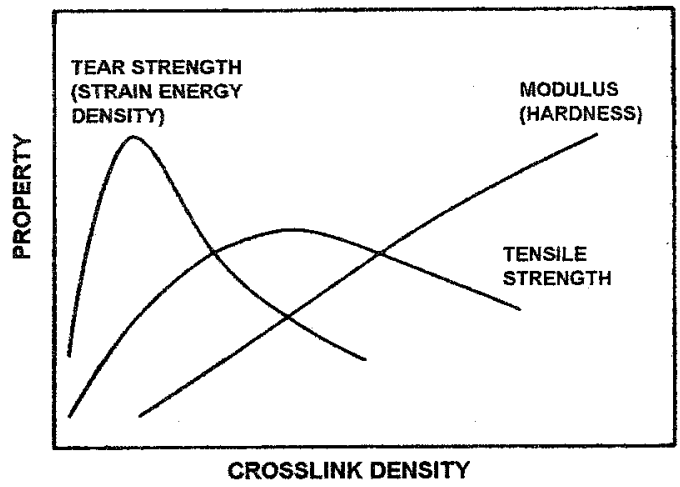

Figure 3.3: Tear strength as a function of crosslink density

\subsection{Polyurethane Microstructure}

Polyurethane has a different physical structure than cross-linked natural rubber and it is important to describe the structure in order to validate our constitutive equation for the finite element analysis.

The polymerization of polyurethane components is described as a reaction of disocynate groups with low molecular weight reagents leading to chain extension and the formation of hard segments connecting the polyether (PTMEG) macroglycol, soft segments through urethane groups.

The term polyurethane is misleading as the main chain is actually composed of 3 main monomers and has a minority of urethane in the chain. Figure 3.4 shows the molecular architecture of Biomer, a biomedical polyurethane. The main components of Biomer are:

- macroglycol

- isocyanate source

- chain extender, curative

Polyurethanes are also block co-polymers meaning that they contain sections of the main chain with low molecular weight macroglycol and are linked together by a urethane group. 


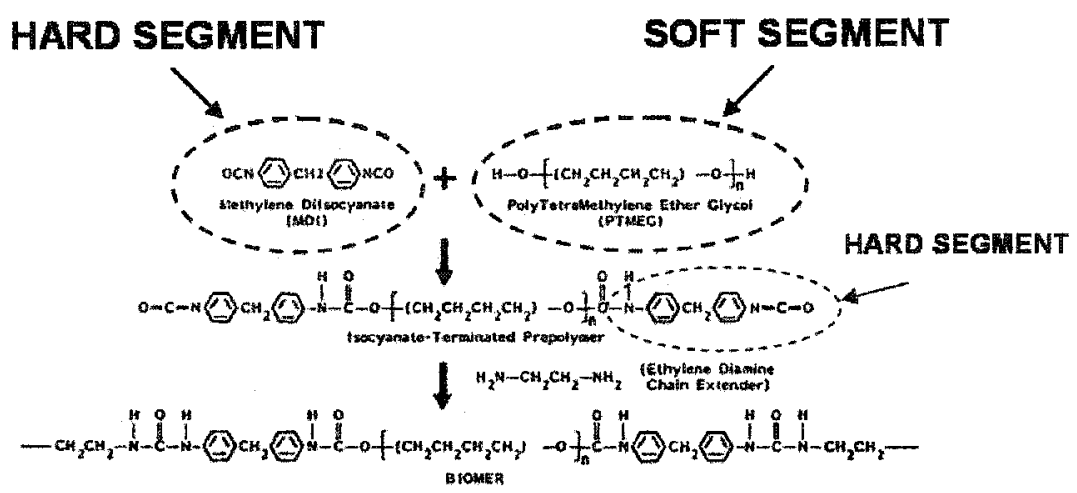

Figure 3.4: Molecular architecture of Biomer

\begin{tabular}{|l|l|l|}
\hline Phase & Macrostructure & Chemical Formulation \\
\hline hard & crystalline & Isocyanate Source (Polyisocyanate) \\
\hline soft & amorphous & Macroglycol (Polyol) \\
\hline
\end{tabular}

Table 3.1: Hard and soft segments and their chemical formulation

\subsection{Polyurethane Macrostructure}

Polyurethanes are observed to have a two phase macrostructure of both crystalline and amorphous regions as shown in Figure 3.5. This is known as segmentation or semicrystalline. Crystalline regions, called hard segments, are formed by the isocyanate source, while the amorphous region, called the soft segment, is formed by a macroglycol. The presence of both macroglycol and isocynate source in the main chain results in soft and hard regions of the material.

The hard segment weight percentage is calculated simply as the weight of isocyanate + chain extender and the weight percentage of soft segment is calculated as the weight of the macroglycol.

It is important to note that, controlling the amount of hard segment in the material improves the biocompatibility of the material as well as increasing the hardness and stiffness of the material. Also, the low molecular weight soft segment is currently being investigated as a source for degradation and attempts have been made to improve this 


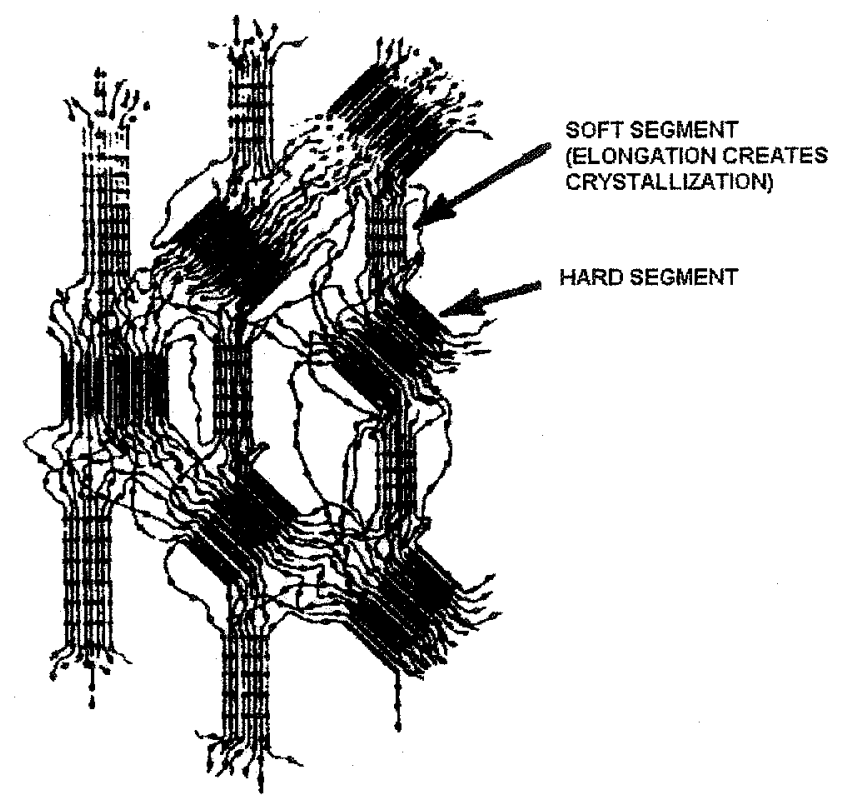

Figure 3.5: Segmented polyurethane elastomer: hard and soft segments

particular portion of the chain without sacrificing other properties.

\subsection{Molecular Interaction During Stress}

Polyurethanes are virtually cross-linked material, meaning that they are not actually crosslinked as explained above. The urethane and urea bonds created from the combination of the macroglycol and diisocynate groups make a secondary level of molecular interaction [26]. These secondary bonds can be broken and reformed an infinite number of times and have a hydrogen bond character. They are approximately $1 / 20$ th of the normal covalent bond strength. This ability to be formed and broken creates a polymer with high fatigue resistance. Similar to vulcanized natural rubber, virtual cross-linked polymers also exhibit a high level of thermoset nature, thus they cannot be thermally processed. They are typically synthesized and processed as a solution using the procedure of dip coating or casting. 
Upon uniaxial extension shown in Figure 3.4, hard segments act as crosslinks between the soft segments. Thus the hard segment content is used as a measure of crosslink density in polyurethanes. At high elongation, $>100 \%$, the soft segments become more crystalline.

\subsection{Molecular Theory of Rubber Elasticity}

Using theories developed in statistics and thermodynamics a stress-strain relationship can be developed for elastomers. The theories are applied to the Gaussian Network Theory of polymer chains to provide a force-extension model which works well in predicting stress in crosslinked natural rubber up to a stretch less than two times its original length. This model is referred to as the Mooney-Rivlin model. The following is the force extension relationship for rubber in uniaxial strain:

$$
\frac{\sigma}{2\left(\lambda-\frac{1}{\lambda^{2}}\right)}=C_{10}+\frac{C_{01}}{\lambda}
$$

where $C_{10}$ and $C_{01}$ are constants. It has been proposed that $C_{10}=\frac{1}{2} N K T$, where $N$ is the number of crosslinks per unit area, $T$ is the temperature, $K$ is the Boltzman constant, and $\lambda$ is the stretch in the material. A complete derivation of this model can be found in [38].

A constitutive equation, derived independently in section 4.4, and satisfying the constraints of continuum mechanics theory fortuitously appears in the same form as the Mooney-Rivlin model. Thus, in finite elasticity theory, it is also known as the MooneyRivlin model, and is shown in Equation 4.32. It is important to note that the constants, $C_{10}$ and $C_{01}$ in the finite elasticity theory are actually derivatives of the strain energy density with respect to the stretch ratios under the strain being considered.

With a strain energy model based on morphological considerations based on the Guassian Network theory, and another strain energy model, derived independently using the 
finite elasticity theory, the constants that characterize a particular material have direct physical significance. This is particularly advantageous as will be discussed later in Chapter 4 .

It is important to state here that $C_{10}$ and $C_{01}$ have a slightly different meaning in polyurethane analysis versus traditional sulphur crosslinks of the type shown in Figure 3.2. Crosslinks in polyurethanes are the result of the hard segment aggregates. The hard segment regions shown in Figure 3.5 act as large polyfunctional crosslinks because long flexible elastomeric sequences, soft segments, are linked together by them also shown in Figure 3.5. Thus, $C_{10}$ in polyurethanes is related to crosslink density through the hard segment content.

Another characteristic of polyurethanes is the phase interaction, which involves secondary bonding between the hard and soft segments. This secondary bonding seems to affect the $C_{01}$ value. It is observed that if hard segment content is increased, the more phase isolation occurs, ie. more distinct regions of hard and soft segments. The values of both $C_{10}$ and $C_{01}$ increases. However, as soft segment molecular weight is increased, the $C_{01}$ value is reduced and this may be the result of a loss in phase interaction, as observed by Benson and Kim [12]. Thus $C_{01}$ change in value is related to this phase separation or breakage of secondary bonding in the region between the two phases.

It is known that as strain occurs in uniaxial tension, hard segments orient themselves in the direction of straining [39], as shown in Figure 3.5. Thus, it appears that this phase interaction is dependent on the type of strain induced as will be discussed later in section 4 when comparison between uniaxial and biaxial straining is compared.

It is observed that with increasing hard segment content, the fatigue crack propagation behaviour improves in biomedical grade polyurethanes [12]. This is also in accordance with Figure 3.3.

Section 4.7 details the steps taken to determine the material constants for this model. 
Although the Mooney-Rivlin model can deliver good results, the material parameters must be very well known to a high degree of accuracy [40] and it is proposed by this study that special considerations should be made for polyurethanes in order to apply the MooneyRivlin model. Also provided in Chapter 4 is a brief description of the governing laws of continuum mechanics from which this equation and the variational function is derived. 


\section{Chapter 4}

\section{Finite Deformation Elasticity}

Biomedical grade elastomeric polyurethane possesses rubber-like characteristics and can be classified as a non-linear elastic solid material. The loading and unloading paths of the stress-strain curve is non-linear and thus a linear function cannot be applied to adequately describe stress response. ABAQUS uses strain energy, the area under the highly curved stress-strain graph, to calculate stresses in non-linear materials. The Mooney-Rivlin coefficients of the last section can be thought of as the elastic moduli of rubber, just like the elastic modulus for steel, where the material stiffness is dependent on the crosslink density (the hard segment content in polyurethane), and the degree of secondary interaction of the chains.

The finite element analysis to solve large deformations in the diaphragm inversion, is also a non-linear problem. During deformation, the initial and final points of the finite element nodes are widely separated. The analysis of such displacements requires the use of non-linear equations from general finite elasticity theory versus the infinitesimal strain theory often used with metal analysis. Thus, two ways rubber elasticity analysis differs from metal elasticity analysis is that the material is non-linear and the analysis method is a non-linear problem. 
The following sections provide a brief review of general finite elasticity theory and the conservation laws of continuum mechanics used to develop the finite element variational function. Later sections discuss how the Mooney-Rivlin coefficients are determined from stress-strain experiments.

\subsection{Measures of Strain}

As the diaphragm material undergoes a deformation, the particles of that continuum approximated by the nodes or integration points of our mesh, will move through space. This can be expressed by:

$$
x_{i}=x_{i}\left(X_{1}, X_{2}, X_{3}, t\right)=x_{i}(\mathbf{X}, t) \text { or } \mathbf{x}=\mathbf{x}(\mathbf{X}, t)
$$

where $x_{i}$ represents the present location of the node or integration point that occupied the original point $\left(X_{1}, X_{2}, X_{3}\right)$ at $t=0$. This can be interpreted as a mapping of the initial configuration into the current configuration. Such a mapping is:

- one to one

- continuous

- has continuous partial derivatives to the required order

The description of motion or deformation given by Equation 4.1 is known as the Lagrangian formulation and is used in non-linear software such as ABAQUS. The mesh that is applied to the diaphragm is attached to it and, thus, deforms with the material. The new nodal locations are defined by referencing them to their undeformed configuration.

By convention, quantities associated with the original point, undeformed condition, are capitalized. 
The differential length of the position vector in its original state is given by:

$$
d X=d X_{A} I_{A}
$$

and similarly for the deformed state:

$$
d x=d x_{i} i_{i}
$$

where $I_{A}$ and $i_{i}$ are the basis vectors in original and deformed configuration, respectively.

The differential quantity $d x_{i}$ can be expressed as:

$$
d x_{i}=\frac{\partial x_{i}}{\partial X_{A}} d X_{A}=x_{i, A} d X_{A}
$$

where $x_{i, A}$ is also known as the deformation gradient tensor $F_{i, A}$ or $\mathbf{F}$.

The deformation gradient tensor is a product of the symmetric positive definite right stretch tensor $U$, orthogonal rotation tensor, $R$ as in, $\mathbf{F}=R U$. The strain measure that is selected must be independent of this rigid body rotation hence the following strain tensor is considered:

$$
(d s)^{2}-(d S)^{2}=\left(d x_{i}\right)^{2}-\left(d X_{A}\right)^{2}=2 d X_{A} E_{A B} d X_{B}
$$

By replacing $d x_{i}$ with the deformation gradient tensor definition in 4.2 :

$$
\begin{gathered}
d X_{A} F_{i, A} F_{i, B} d X_{B}-\delta_{A B} d X_{A} d X_{B}=2 d X_{A} E_{A B} d X_{B} \\
d X_{A}\left(F_{i, A} F_{i, B}-\delta_{A B}\right) d X_{B}=2 d X_{A} E_{A B} d X_{B}
\end{gathered}
$$

where $\delta_{A B}$ is the Kroenecker delta. The strain tensor can be written in terms of the 
deformation gradient tensor, and, as noted, independent of the rotation tensor. This is known as the Green-Lagrangian deformation tensor:

$$
E_{A B}=\frac{1}{2}\left(F_{i, A} F_{i, B}-\delta_{A B}\right)=\frac{1}{2}\left(C_{A B}-\delta_{A B}\right)
$$

where $C_{A B}$ is known as the right Cauchy deformation tensor, or Green deformation tensor and is often used to help define constitutive equations for non-linear (large deformation) solid mechanics.

Using polar decomposition theory, the deformation tensor, $\mathrm{C}$, is related to the symmetric stretch tensor, $\mathrm{U}$ by the orthogonal rotation tensor, $R^{T}=R^{-1}$ :

$$
\mathbf{C}=\mathbf{F}^{T} \mathbf{F}=(R U)^{T}(R U)=U^{T} R^{T} R U=U^{T} U=U^{2}
$$

As noted, during deformation, the body may rotate as well as stretch. As the body is rotating, there exists scalar values of $\mathbf{C}$, representing a given stretch, that will not vary. These are known as the invariants, or scalar combinations of the components of $\mathbf{C}$.

First it is necessary to calculate the $\mathrm{C}=F^{T} F$ matrix. The eigenvalues are roots of the characteristic polynomial of $\mathbf{C},\left(\left(\lambda_{1}\right)^{2},\left(\lambda_{2}\right)^{2},\left(\lambda_{3}\right)^{2}\right)$ and the eigenvectors $\left(s_{1}, s_{2}, s_{3}\right)$ can be determined from each eigenvalue.

The stretch ratio is given by:

$$
\lambda=\frac{l_{s}}{l_{o}}
$$

stretched gauge length on a test specimen

where $l_{s}$ is the stretched gauge length on a test specimen and $l_{0}$ is the original length. It is convenient to use this form of strain measure in large deformation analysis as it is more convenient mathematically. 
The Cauchy deformation tensor in terms of stretch is given by,

$$
\mathbf{C}=\Omega^{T} \Lambda^{2} \Omega=\Omega^{T}\left[\begin{array}{ccc}
\left(\lambda_{1}\right)^{2} & 0 & 0 \\
0 & \left(\lambda_{2}\right)^{2} & 0 \\
0 & 0 & \left(\lambda_{3}\right)^{2}
\end{array}\right] \Omega
$$

and, $U$, the stretch tensor, can be shown to be as follows by Equation 4.4:

$$
\mathbf{U}=\Omega^{T} \Lambda \Omega=\Omega^{T}\left[\begin{array}{ccc}
\left(\lambda_{1}\right) & 0 & 0 \\
0 & \left(\lambda_{2}\right) & 0 \\
0 & 0 & \left(\lambda_{3}\right)
\end{array}\right] \Omega
$$

The columns of $\Omega$ are the eigenvectors that represent the orientation of the principal stretch axes with respect to the material coordinates and $\lambda_{i}$ are the principal stretches. Organizing $\mathbf{C}$ by means of the similarity transformation results in $\mathbf{C}$ being diagonalized.

The principal invariants are scalar quantities of $\mathbf{C}$, and are given in Equation( 4.6):

$$
I_{1}=\operatorname{tr}(\mathbf{C}) \quad I_{2}=\frac{1}{2}\left[(\operatorname{tr}(\mathbf{C}))^{2}-\left(\operatorname{tr} \mathbf{C}^{2}\right)\right] \quad I_{3}=\operatorname{det}(\mathbf{C})
$$

where $\operatorname{tr}(\mathbf{C})$ is the sum of $\mathbf{C}_{m m}$. Using the similarity transformation above, it is convenient to express the invariants in terms of the principal invariants.

The invariants of the deformation

$$
\begin{aligned}
& I_{1}=\left(\lambda_{1}\right)^{2}+\left(\lambda_{2}\right)^{2}+\left(\lambda_{3}\right)^{2} \\
& I_{2}=\left(\lambda_{1}\right)^{2}\left(\lambda_{2}\right)^{2}+\left(\lambda_{2}\right)^{2}\left(\lambda_{3}\right)^{2}+\left(\lambda_{3}\right)^{2}\left(\lambda_{1}\right)^{2} \\
& I_{3}=\left(\lambda_{1}\right)^{2}\left(\lambda_{2}\right)^{2}\left(\lambda_{3}\right)^{2}
\end{aligned}
$$

The Mooney-Rivlin model of strain energy, is an invariant function. Meaning that it 
must be a function of the invariants shown above in Equation 4.7. It is possible to develop an equation using linear elastic theory that is in the same form as Equation 3.1, since the invariants can be defined in terms of stretch and, also, when the uniaxial strain state is considered. Rearranging Equation 3.1 into a form representing the equation of a line, it is possible to determine $C_{10}$ and $C_{01}$, the Mooney-Rivlin coefficients, characteristic of the material being used. These coefficients can be thought of as a coefficients such as the elastic modulus for steel. In Section 4.4, Equation 3.1 is derived in terms of stretch by means of the continuum theory and by applying the constraints of invariance and incompressibility. First it is necessary to define the measures of stress that will incorporate the Mooney-Rivlin function of strain energy density.

\subsection{Measures of Stress}

The previous section defined measures of strain in terms of large deformations. It is convenient to express stress in terms of large deformations as well. This is necessary for finite element implementation and to incorporate the Mooney-Rivlin form of strain energy density. Rather than to showing detailed equations, the approach taken to explain this aspect of large deformation theory is more descriptive. For a complete mathematical derivation refer to [16]. The Cauchy stress tensor is a second order tensor of stress vectors defined as the true force/unit deformed area and is as follows:

$$
\sigma_{i j}=\left(\begin{array}{ccc}
\sigma_{11} & \sigma_{12} & \sigma_{13} \\
\sigma_{21} & \sigma_{22} & \sigma_{23} \\
\sigma_{31} & \sigma_{32} & \sigma_{33}
\end{array}\right)
$$

The strain tensor that is used with the Cauchy stress tensor is the infinitesimal strain tensor for small deformations. Given that in large deformation analysis, the deformed area 
is not always known, thus it is appropriate to derive the stress measure with respect to the reference configuration.

The strain energy determined by the Cauchy stress tensor and the small deformation tensor should be equivalent to a strain energy determined for large deformation analysis.

$$
\sigma_{i j} \epsilon_{i j}=T_{A B} E_{A B}
$$

where, $E_{A B}$ is the Green-Lagrangian strain tensor derived in the previous section, for large deformations, and $T_{A B}$ is a stress measure called $2^{\text {nd }}$ Piola-Kirchoff $\left(2^{\text {nd }} \mathrm{P}-\mathrm{K}\right)$ stress tensor.

The derivation of $T_{A B}$ benefits finite element simulation as this stress measure is symmetric. Furthermore as shown in Equation 4.9, the tensor is energetically consistent with Green-Lagrange strain, ie. it can be multiplied by the Green-Lagrangian strain to determine the strain energy. For a complete derivation of the $2^{\text {nd }}$ Piola-Kirchoff stress tensor please refer to $[16]$.

In summary, the $2^{\text {nd }}$ Piola-Kirchoff $(P-K)$ stress tensor, denoted $T_{A B}$, represents the force measured per unit undeformed area acting on an element of surface in the original configuration. This stress tensor is completely defined with respect to the material coordinates as in the case of the Green-Lagrangian strain tensor.

Finally, a third stress measure, mentioned for completeness, is the $1^{\text {st }}$ Piola-Kirchoff stress tensor, denoted $s_{A j}$. It is similar to that of the $2^{\text {nd }}$ second P-K tensor in that it represents the force measure per unit undeformed area except now it is acting on an element of surface in the deformed configuration; ie. it is spatially referenced. In other words, the first index represents the normal of the surface in the undeformed case, and is hence, referring to the material coordinates, and the second index represents the direction of force on the deformed material. The result is an unsymmetrical matrix. The stress tensor is also known as engineering stress as in some mechanical experiments where stress 
is calculated for per unit undeformed area.

The $1^{\text {st }} \mathrm{P}-\mathrm{K}$ tensor is mentioned as the following laws of continuum mechanics and the resulting equation, require a spatial frame of reference. This is later converted to the $2^{\text {nd }}$ P-K tensor when a symmetric matrix is more suitable for finite element formulation.

\subsection{Equilibrium Laws of Continuum Mechanics}

For finite element analysis, a variational function is developed that encompasses the stress-strain relationship in three dimensions. Using the fundamental laws of continuum mechanics a suitable function relating the body deformation will be developed. The aim is to write the equations of motion in terms of the spatially defined Cauchy's stress tensor. The $2^{\text {nd }} \mathrm{P}-\mathrm{K}$ tensor can then be substituted for the purpose of FEA simulation. Once the equations of motion are established and the laws of continuum mechanics upheld, the principle of virtual work or conservation of energy is applied to the function.

\subsubsection{Conservation of Mass}

The law of conservation of mass requires that the mass of a specific portion of the continuum remain constant. If $V_{o}$ is the volume of the integral at time $t=0$;

$$
\int_{V_{o}} \rho_{o} d V_{o}=\int_{V_{o}} \rho J d V_{o}
$$

where $\mathrm{J}$ is the Jacobian matrix, $V_{o}$ is the volume of the continuum at time $t=0$, and $\rho_{o}$ is the density of continuum at time $t=0$. 


\subsubsection{Conservation of Linear Momentum}

Following Mase [41], based upon Newton's second law, the principle of linear momentum states that the time rate of change of total linear momentum of an arbitrary portion of a continuum is equal to the resultant forces acting upon the considered portion. The principle in integral form is:

$$
\int_{S} t d S+\int_{V} \rho b d V=\frac{d}{d t} \int_{V} \rho v d V
$$

where $t$ is the traction vector, $b$ represents the body forces (per unit mass) and $v$ is the velocity field, $S$ is the external surface loaded by the traction vectors not subjected to boundary conditions.

As was noted earlier the equilibrium equation must be reformulated in terms of the $1^{\text {st }}$ P-K stress tensor or Cauchy tensor, as the laws and the equations are spatially defined. The surface traction can be expressed in terms of the rectangular coordinate system, and thus Cauchy's tensor, by Cauchy's formula:

$$
t d S=\sigma_{i j} \hat{n}_{i} i_{j} d S
$$

where $i_{j}$ and $\hat{n}_{i}$ are the unit normal vectors to the surface.

Substituting Cauchy's stress tensor:

$$
\int_{S} \sigma_{i j} \hat{n}_{i} d S=0
$$

After applying the divergence theory to the surface integral:

$$
\int_{V} \frac{\partial \sigma_{i j}}{\partial x_{i}}=0
$$


Finally substituting for the $2^{\text {nd }} \mathrm{P}-\mathrm{K}$ tensor:

$$
\frac{\partial}{\partial X_{A}}\left(T_{A B} \frac{\partial x_{j}}{\partial X_{B}}\right)=0
$$

The above expression is the variational function of the conservation of energy due to motion by the body deformation.

\subsubsection{Conservation of Angular Momentum}

The conservation of angular momentum is ignored as the analysis is quasi-static. Should any change in external force occur, this must satisfy the principle of virtual work which conserves energy in the system.

\subsubsection{Principle of Virtual Work (Conservation of Energy)}

If the body undergoes an arbitrarily small displacement, $\delta v$, and satisfies compatibility and any displacement boundary conditions, then the principal of virtual work can be expressed as:

$$
\int_{S_{x}} s \cdot \delta v d S=\int_{S} t \cdot \delta v d S
$$

where $S$ is the external surface loaded by the surface tractions, $S_{x}$ is the portion of surface not subjected to boundary conditions, $t$ is the internal stress vector, $s$ is the external stress vector and $\delta v$ are arbitrarily small displacements.

The principle of virtual work incorporates the conservation of energy in the system. It is this equation that will lead to the variational function upon which the finite element method is based. 
The aim is to put the above equation in terms of the $2^{\text {nd }} \mathrm{P}-\mathrm{K}$ stress tensor. Substituting Cauchy's formula from Equation 4.12 for the internal stress vector:

$$
\int_{S_{x}} s \cdot \delta v d S=\int_{S} \sigma_{i j} \hat{n} \delta v_{j} d S
$$

Using Gauss' theorem, the right hand side of the above expression can be expressed as a volume integral.

$$
\int_{S_{x}} s \cdot \delta v d S=\int_{V}\left[\frac{\partial \sigma_{i j}}{\partial x_{i}} \delta v_{j}+\sigma_{i j} \frac{\partial \delta v_{j}}{\partial x_{i}}\right] d V
$$

By substituting Cauchy's first law of motion 4.11 and substituting for the $2^{\text {nd }} \mathrm{P}-\mathrm{K}$ stress tensor, the following is the equation for static equilibrium:

$$
\int_{S_{x}} s \delta v d S=\int_{V} T_{A B} \frac{1}{J} \frac{\partial x_{j}}{\partial X_{A}} \frac{\partial \delta v_{j}}{\partial X_{B}} d V
$$

It remains to determine the expression for the surface tractions in terms of the boundary conditions.

\subsection{Neumann and Dirichlet Boundary Conditions}

With the governing equations established, it remains to define the external forces on the system on the left hand side of Equation 4.19 involving, the surface traction vector $s$.

The external pressures, that are applied, are evaluated along the surface sustaining the load. As was mentioned in Chapter 2, the $\triangle P$ can be be applied to one side of the diaphragm. The surface affected is denoted $S_{x}$ with unit normal $\hat{n}$. If the surface is loaded by a pressure, $P_{\text {fluid }}$ (hydraulic fluid), then the surface traction vector has components $s=P_{\text {fluid }} \hat{n}$

Substituting into Equation 4.19 yields the governing equations for finite deformation 
elasticity with respect to rectangular cartesian coordinates:

$$
\int_{S_{x}} P_{(\text {fluid })} \hat{n}_{j} \delta v d S=\int_{V} T_{A B} \frac{1}{J} \frac{\partial x_{j}}{\partial X_{A}} \frac{\partial \delta v_{j}}{\partial X_{B}} d V
$$

The above equation must still be solved in terms of the virtual displacements $d v_{j}$ and any boundary conditions of that displacement. Before proceeding further, it is appropriate to show how the constitutive equation is determined for a rubber-like or hyperelastic material and apply it to our governing equation.

\subsection{Constitutive Equation}

The $2^{\text {nd }} \mathrm{P}-\mathrm{K}$ stress tensor is a function of the strain energy potential. The strain energy is a function of stress and strain. The following section involves the derivation of a stress equation in terms of uniaxial stretches. The equation of stress is a function of the invariants and incorporates the constraint of incompressibility for rubber; these constraints are explained later. The derived stress-strain curve is fit to standard uniaxial tensile data by adjusting the coefficients of the curve. The characteristic coefficients for Material \#1 in uniaxial tension are then determined. The same approach can be taken with biaxial and pure shear stress-strain data. The same coefficients of the stress-strain curves for biaxial and uniaxial case are then used in the equation of strain energy potential. Thus, with the $2^{\text {nd }} \mathrm{P}-\mathrm{K}$ measure of stress fully described in terms of the strain energy, the stresses in the diaphragm under different modes of deformation are determined in the finite element analysis.

The final expression for the $2^{\text {nd }}$ P-K measure of stress is as follows:

$$
T_{A B}=\frac{1}{2}\left(\frac{\partial W(\mathbf{C})}{\partial E_{A B}}+\frac{\partial W(\mathbf{C})}{\partial E_{A B}}\right)
$$


where $E$ is the Green-Lagrange Strain Tensor, and $T$ is the $2^{\text {nd }} \mathrm{P}-\mathrm{K}$ strain tensor. $E$ is written in terms of the principal stretch ratios and $W(\mathbf{C})$ is an invariant function.

Hyperelastic materials are any material that can be expressed in terms of a strain energy potential. Strictly speaking, all materials fall into this category, however, only a minority of materials require an equation in terms of strain energy to sufficiently describe them in finite element analysis. Hence, in practice, this term is often used for materials such as rubber.

The strain energy can then be written as:

$$
W\left(I_{1}, I_{2}, I_{3}\right)
$$

where $W$ is now written as a function of the invariants. $W$ can be any arbitrary function and a suitable choice would be a power series expansion in terms of the invariants, $I_{1}, I_{2}$ and $I_{3}$ as shown in Equation 4.23.

$$
W\left(I_{1}, I_{2}, I_{3}\right)=\sum_{i=0, j=0, k=0}^{\infty} C_{i j k}\left(I_{1}-3\right)^{i}\left(I_{2}-3\right)^{j}\left(I_{3}-1\right)^{k}
$$

where $C_{i j k}$ are material constants. This form satisfies the zero stress at zero deformation principle in continuum mechanics as when $I_{1}=I_{2}=3$ and $I_{3}=1$ for all $\lambda_{i}=1$. Also, $W$ is an invariant function, ie. the strain energy does not change with respect to any co-ordinate system, it must be a function of the invariants.

It is important to note that the coefficients of Equation 4.23 are derivatives of the strain energy function with respect to the invariants for example,

$$
C_{100}=\frac{\partial W}{\partial I_{1}}
$$

With the invariants, $I_{i}$, in terms of the principal stretch ratio, an expression can be derived from which it is possible to obtain characteristic information for a given material 
type using a uniaxial tensile test.

Let us consider work on the body stored in the form of strain energy for an isothermal deformation. The rate of change of the energy with respect to any strain is the corresponding stress.

$$
d W=\sigma_{11} \lambda_{2} \lambda_{3} d \lambda_{1}+\sigma_{22} \lambda_{1} \lambda_{3} d \lambda_{2}+\sigma_{33} \lambda_{1} \lambda_{2} d \lambda_{3}
$$

where $W$ is the strain energy per unit volume. If we consider only one direction, with reference to Equation 4.24, we see that $\sigma_{11} \lambda_{2} \lambda_{3} d \lambda_{1}$ is the work done in direction 1 . Rearranging the expression we get the stress in direction 1 as:

$$
\sigma_{11}=\frac{1}{\lambda_{2} \lambda_{3}} \frac{\partial W}{\partial \lambda_{u}}
$$

where the rate of change of $W$ with respect to the stretch in the direction of loading $\lambda_{1}=\lambda_{u}$, can now be written as:

$$
\frac{\partial W}{\partial \lambda_{u}}=\frac{\partial W}{\partial I_{1}} \frac{\partial I_{1}}{\partial \lambda_{u}}+\frac{\partial W}{\partial I_{2}} \frac{\partial I_{2}}{\partial \lambda_{u}}+\frac{\partial W}{\partial I_{3}} \frac{\partial I_{3}}{\partial \lambda_{u}}
$$

where $\lambda_{u}$ is the stretch in the loading direction and $I_{1}, I_{2}$ and $I_{3}$ are the principal invariants and are defined in Equation 4.7.

Substituting equations 4.26 into 4.25 , the resulting equation can be used to extract the characteristic coefficients to describe the strain energy conserved under uniaxial tension.

At this point we introduce a necessary constraint for hyperelastic materials that redefines the principal invariants of Equation 4.7. Polyurethane is assumed to be incompressible. This means that the volumetric deformation is assumed to be zero. For example, if a rubber ball is placed at the bottom of the ocean, the enormous hydrostatic pressure, 
will have no effect on the compression or volume change in the ball, hence there is no work done on the material. This must be accounted for in our governing equation. If it is assumed the volume does not change during deformation, then $\lambda_{1}, \lambda_{2}$, and $\lambda_{3}$ are not independent and thus:

$$
\lambda_{1} \lambda_{2} \lambda_{3}=1
$$

If the incompressibility constraint, $I_{3}=1$ is introduced, the Equations 4.7 are reduced to:

$$
\begin{aligned}
& I_{1}=\left(\lambda_{1}\right)^{2}+\left(\lambda_{2}\right)^{2}+\left(\lambda_{3}\right)^{2} \\
& I_{2}=\frac{1}{\left(\lambda_{1}\right)^{2}}+\frac{1}{\left(\lambda_{2}\right)^{2}}+\frac{1}{\left(\lambda_{3}\right)^{2}} \\
& I_{3}=1
\end{aligned}
$$

The new strain energy with the incompressibility constraint is:

$$
W\left(I_{1}, I_{2}\right)
$$

Equation 4.26 also reduces to:

$$
\frac{\partial W}{\partial \lambda_{u}}=\frac{\partial W}{\partial I_{1}} \frac{\partial I_{1}}{\partial \lambda_{u}}+\frac{\partial W}{\partial I_{2}} \frac{\partial I_{2}}{\partial \lambda_{u}}
$$

Returning back to the case of uniaxial tension, the principle extension ratios for the test in uniaxial tension are shown in Figure 4.1.

The principal extension ratios in simple extension, show that:

$$
\lambda_{1}=\lambda_{u}, \lambda_{2}=\lambda_{3}=\frac{1}{\sqrt{\lambda_{u}}}
$$

Now substituting the above extension ratios, into Equation 4.30, the stress in one 


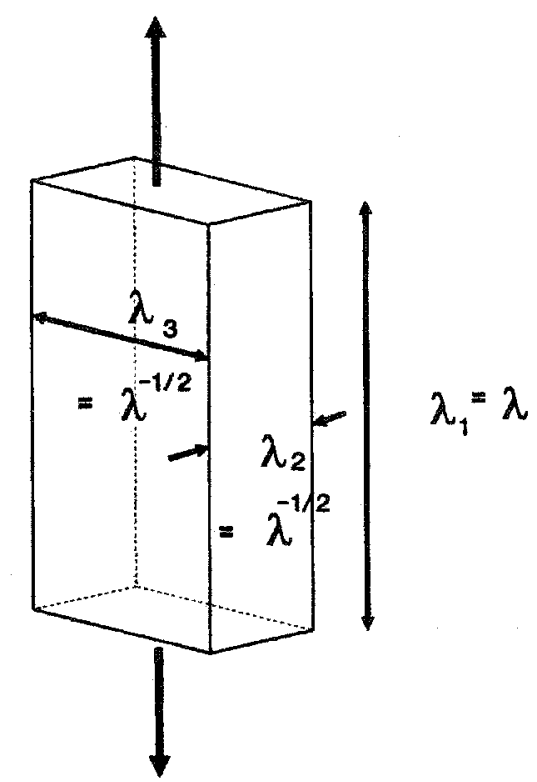

Figure 4.1: Principal extension ratios in simple extension [38]

direction, Equation 4.25 becomes:

$$
\sigma_{11}=\lambda_{u} \frac{\partial W\left(I_{1}, I_{2}\right)}{\partial \lambda_{u}}+P
$$

Now substituting $\frac{\partial I_{1}}{\partial \lambda_{u}}$ and $\frac{\partial I_{2}}{\partial \lambda_{u}}$ (where $I_{1}$ and $I_{2}$ are in terms of the uniaxial tensile case) into 4.30 we arrive at:

$$
\sigma_{11}=\left(\lambda_{u}^{2}-\frac{1}{\lambda_{u}}\right)\left(C_{10}+\frac{1}{\lambda_{u}} C_{01}\right)
$$

where $\sigma_{11}$ is the true stress and $C_{10}$ and $C_{01}$ are left to be determined from uniaxial test data.

Similarly, the principle extension ratios for biaxial tension are shown in Figure 4.2.

The extension ratios, $\lambda_{1}, \lambda_{2}, \lambda_{3}$ that are defined for biaxial deformation in Figure 4.2 are used in the biaxial tension form of the Mooney-Rivlin Equation 4.33. 


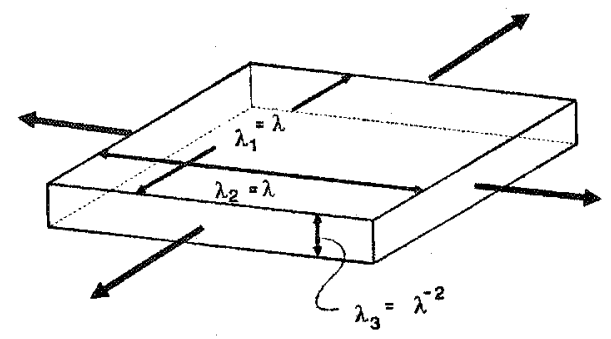

Figure 4.2: Principal extension ratios in equibiaxial extension [38]

$$
\lambda_{1}=\lambda_{2}=\lambda_{u}, \lambda_{3}=\frac{1}{\lambda_{u}^{2}}
$$

Thus, for biaxial extension, the Mooney-Rivlin equation is derived as follows:

$$
\sigma_{11}=\sigma_{22}=2\left(\lambda_{u}^{2}-\frac{1}{\lambda_{u}^{4}}\right)\left(C_{10}+\lambda_{u}^{2} C_{01}\right)
$$

It is important to note that the coefficients of the derived Equation 4.32 are derivatives of the strain energy function with respect to the first and second invariants and are dependent upon the loading condition. Thus,

$$
\begin{aligned}
& C_{10}=\frac{\partial W}{\partial I_{1}} \\
& C_{01}=\frac{\partial W}{\partial I_{2}}
\end{aligned}
$$

Referring now back to Equation 4.23, introducing the incompressibility term, $I_{3}=1$ in the series expansion drops the terms affected by the constraint. From experimentation and prior testing, the first two terms dominate in Equation 4.23 and we come to the popular Mooney-Rivlin two term approximation shown in Equation 4.34. This particular curve fit is verified for use with polyurethane in the following section. Also, Equation 4.34 is used in Equation 4.21 for the finite element implementation. 


$$
W\left(I_{2}, I_{2}\right)=C_{10}\left(I_{1}-3\right)+C_{01}\left(I_{2}-3\right)
$$

The coefficients can be determined from Equations 4.32 and 4.33 from experimental data.

According to ABAQUS, the Mooney-Rivlin form of strain energy potential provides a sufficiently accurate representation up to strains less than 100\% [42]. Other studies show that the Mooney-Rivlin form is commonly used for natural and synthetic rubbers and reproduces behaviour up to moderate deformations $(\lambda<4)$. As will be shown in the next section, the Mooney-Rivlin form of strain energy function is adequate in range of interest for this study, $0-50 \%$ strain for polyurethane.

The use of the Mooney-Rivlin form is particularly advantageous as the uniaxial stress Equation 4.32, is similar to the thermo-statistical model shown in Equation 3.1. With this similarity, a correlation can be made between mechanical properties and the macrostructure of polyurethane. For example, the increase of hard segments increases $C_{10}$. Also, the detachment of hard and soft segments upon stressing affects $C_{01}$ [12]. The use of such a model is advantageous to designers as the coefficients have a physical meaning other than to fit the curve modelling for stress analysis.

\subsection{Application to Diaphragm Design}

Slight differences exist in stress-stretch responses of different material tests and must be accounted for in a truly representative model. For example, uniaxial data alone would not be sufficient to describe the biaxial stretching of the diaphragm during stage 3 nor the biaxial folds in stage 2 as shown in Figure 4.4 
The standard homogeneous tests for material characterization in ABAQUS are:

- Uniaxial Extension

- Biaxial Extension

- Pure Shear

ABAQUS recommends that combining test data from uniaxial tension, uniaxial compression and planar tension (pure shear) provides a good characterization of hyperelastic behaviour. For finite element analysis, if the deformation modes are not known in advance, all tests must be conducted in order to sufficiently describe the material.

It is important to review the primary loading modes in the diaphragm before embarking on developing and testing to obtain material properties in a particular type of strain. LVAD diaphragms are often mounted to their base by an adhesive around the edge as well as being clamped. Typical drawings of this mounting is shown in Figure 2.14. It is expected that the confined edges will also produce stress gradients through the material at or near the edges. These stresses are the result of uniaxial stretching, biaxial stretching and shear as the diaphragm stretches during inversion and during the stretching in late diastole and systole. Pure shear is present particularly at the elements constrained at the edges. Unfortunately, sample data for pure shear is not available at this time as the samples provided for study are not of sufficient size. ABAQUS approximates a response as the uniaxial and biaxial stress response curves represent an upper and lower bound when ABAQUS converges to a solution for a particular deformation mode. Also as shown in standard rubber tests, the pure shear response is between the biaxial and uniaxial response as shown in Figure 4.3. In fact, the designer may wish to use the uniaxial response data for pure shear as a conservative first approximation.

As mentioned earlier, the stresses in endsystolic position, (maximum pressure behind the diaphragm) are biaxial. Biaxial folds, near the edges or across the diaphragm are present and in fact are shown to cause the most failures as found by Zartnack [21]. Uniaxial 


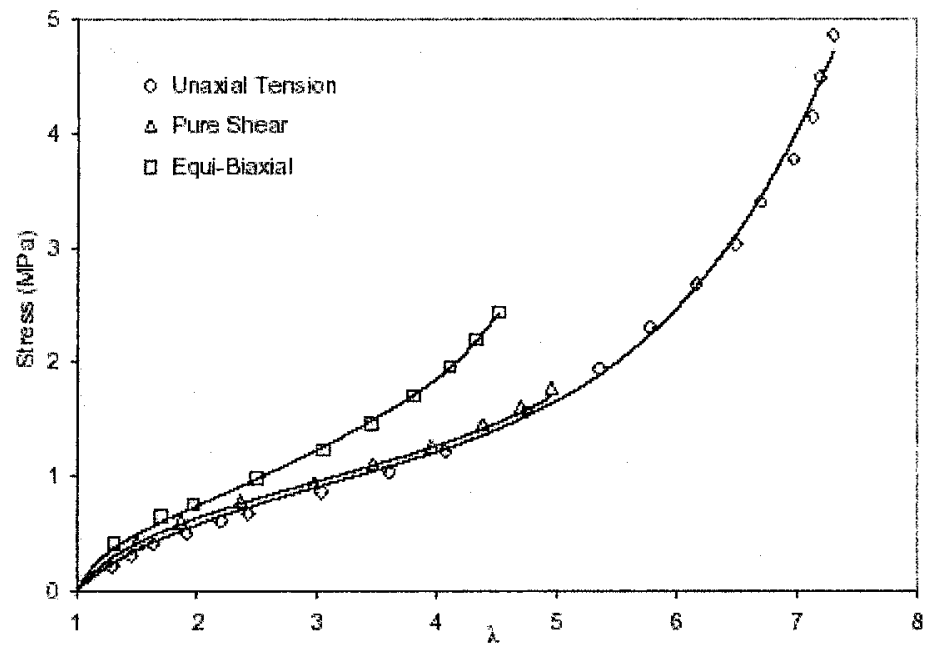

Figure 4.3: Stress-strain response of carbon black rubber in three deformation states $[43]$
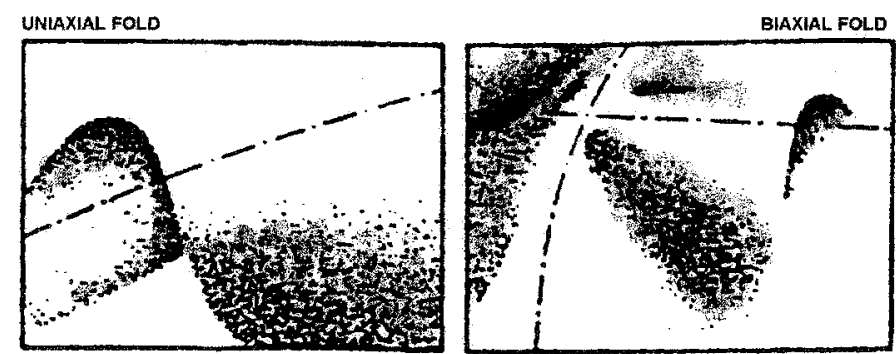

Figure 4.4: Uniaxial and biaxial folding modes [21]

folds are present as well as shown in Figure 4.4.

Thus, to summarize this review of deformation modes, the material tests to determine the Mooney-Rivlin coefficients of Equation 4.34, for this study, are uniaxial and biaxial strain.

\subsubsection{Other Considerations}

Viscoelasticity accounts for the delayed response of a material to an applied stress. The material's viscoelastic response is not modelled in this study. A static analysis is 


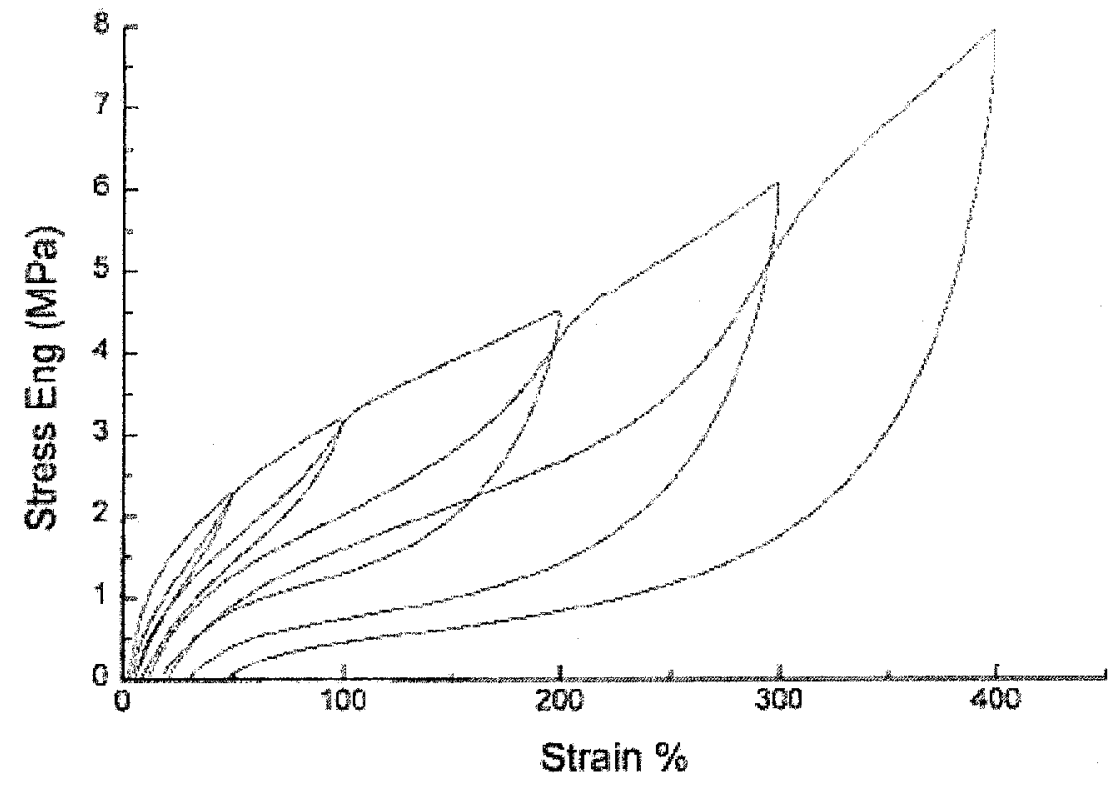

Figure 4.5: Effect of hysteresis on virgin stress-strain curve for Material \#2[39]

undertaken versus a quasi-static option where the user can specify the materials time dependent viscoelastic behaviour. As a static analysis represents the stress distribution, the time delay response is not of interest in this study.

It is also important to mention the effect of the material softening. Upon continuing loading cycles polyurethane materials soften. This is also called the Mullins effect. The effect can be attributed to the disruption of the hydrogen bonds in the hard segment. The hysteresis effect on the stress-strain curve for Material \#2, another clone of Biomer, is shown in Figure 4.5.

During data collection the phenomenon is resolved by first stretching the material up to the maximum strain of interest 3-20 times. The uniaxial and biaxial material tests, described later, used virgin or previously unstrained specimens. Fortunately, for the strain range considered in this study, $<50 \%$, the residual strain due to hysteresis should be less than $5 \%$ as shown in Figure 4.6 for Material \#2 formulations.

Despite the low residual strain in the range of interest for this study, it is possible that 


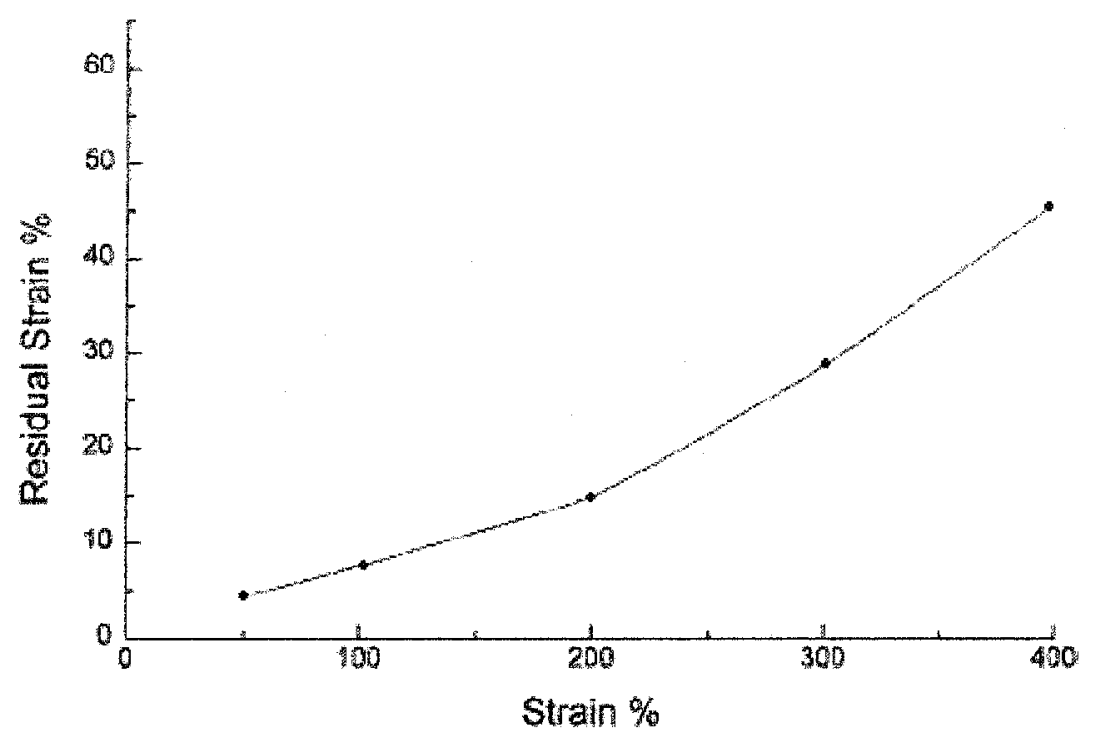

Figure 4.6: Residual strain Material \#2 formulation at varying strains[39]

hysteresis would have some effect on the response of the diaphragm. If the material is softened after the first stretch a lower pressure may be required to invert the diaphragm. Furthermore, the percent hysteresis in the diaphragm for strains up to $50 \%$, is approximately $20 \%$ loss in stress response, for Material \#2. Thus, as the diaphragm inflates during stage 3 , this would result in extra material slack, and perhaps excessive chaffing against the housing. Thus, an analysis for the $20 \%$ hysteresis effect on the stress-strain response is recommended but was not investigated in this study. Furthermore, a residual strain of no less than $5 \%$ should be considered as part of any deflection analysis.

\subsection{Material Properties and Coefficients}

The materials used are believed to be clones of the Biomer formulation shown in Figure 3.4. These materials are labelled Material \#1 and Material \#2. Both materials are compared against data for Biomer in uniaxial tension, and for the biaxial case, only Material \#1 is compared against Biomer. Strain data for Material \#2 in the biaxial case is 
unavailable.

The Mooney-Rivlin coefficients can be obtained from experimental test data of true stress versus true strain. The raw data can be entered into ABAQUS for evaluation. The actual method that ABAQUS uses is verified in the following section using a similar least squares method of fit for both the uniaxial and biaxial cases. The data must be entered in ABAQUS as nominal stress or $\sigma_{N}$ versus stretch.

\subsubsection{Uniaxial Tensile Test}

The uniaxial tensile case is considered first. Once again, this particular stress state shown in Equation 4.32 is similar to Equation 3.1.

The nominal stress-strain response for Material \#1, a proprietary material, was available for strains up to $50 \%$. The data, as provided, contained end effects from testing indicating that the sample slipped or shifted in its clamp. This resulted in a zero stress at a approximately $4 \%$ strain.

DigiMatic, software that records user selected points from a graphic image in an $x-y$ plane, was used to generate a new graph. Data points were selected for strains up to 40\%. The data was then adjusted for zero shift. Each strain point was reduced by the strain value where monotonic stress-strain response begins. With end effects removed, the resulting graph of stress vs. strain is shown in Figure 4.8 .

Uniaxial data for another Biomer clone called Material \#2, was also available. The manufacturers also expressed interest in the research. The data is used for comparison with Material \#1 and is superimposed in Figure 4.8. The full curve of Material \#2 data up to the ultimate tensile strain of $892 \%$, is shown in Figure 4.7 . Material \#1 response is expected also be a non-linear curve up to the ultimate tensile strain as shown by the full curve of Material \#2.

The extension ratios, $\lambda_{1}, \lambda_{2}, \lambda_{3}$ that are defined in Figure 4.1 are used in the uniaxial 


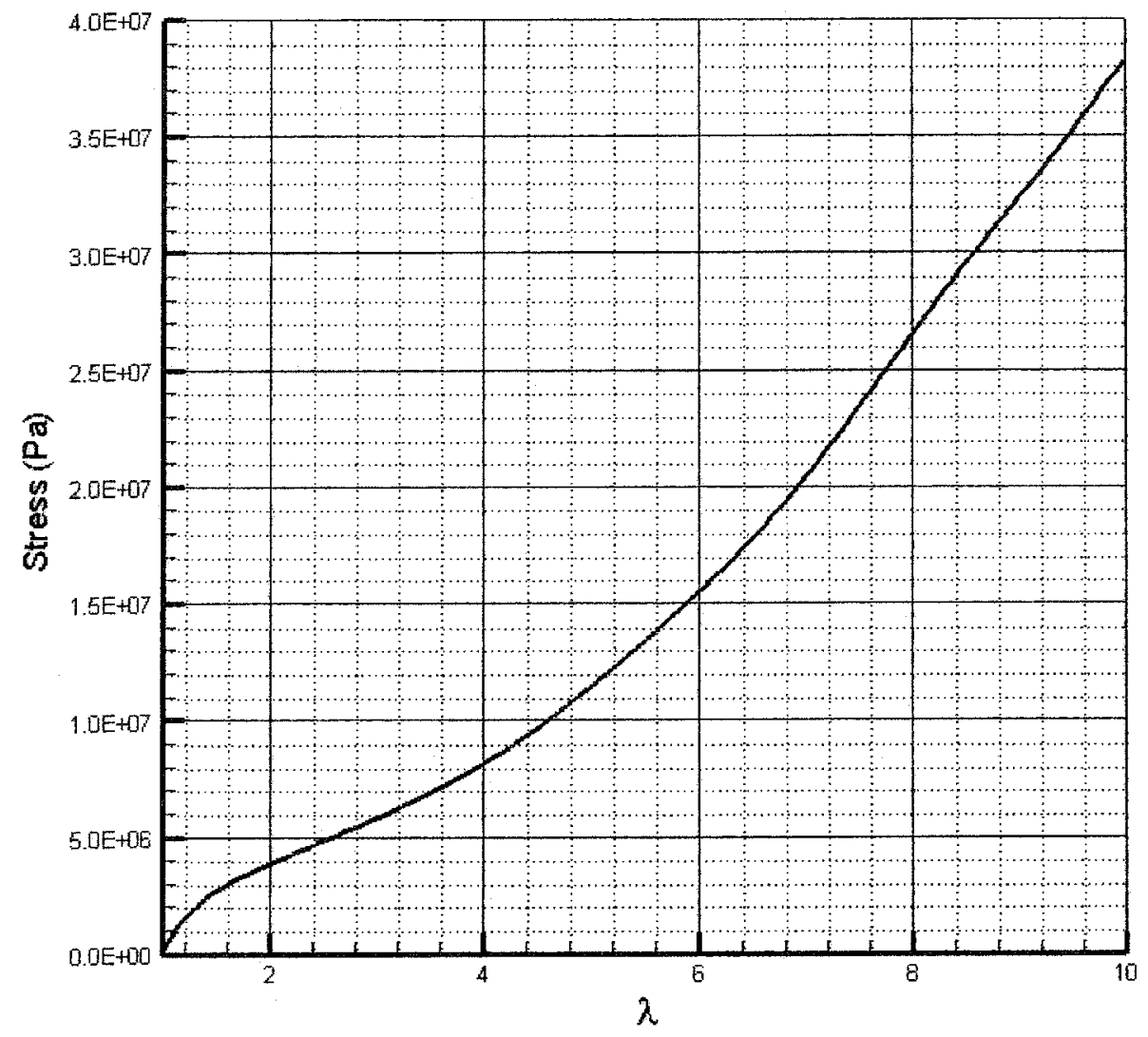

Figure 4.7: Full curve of uniaxial tensile data for Material \#2 (Biomer clone) 


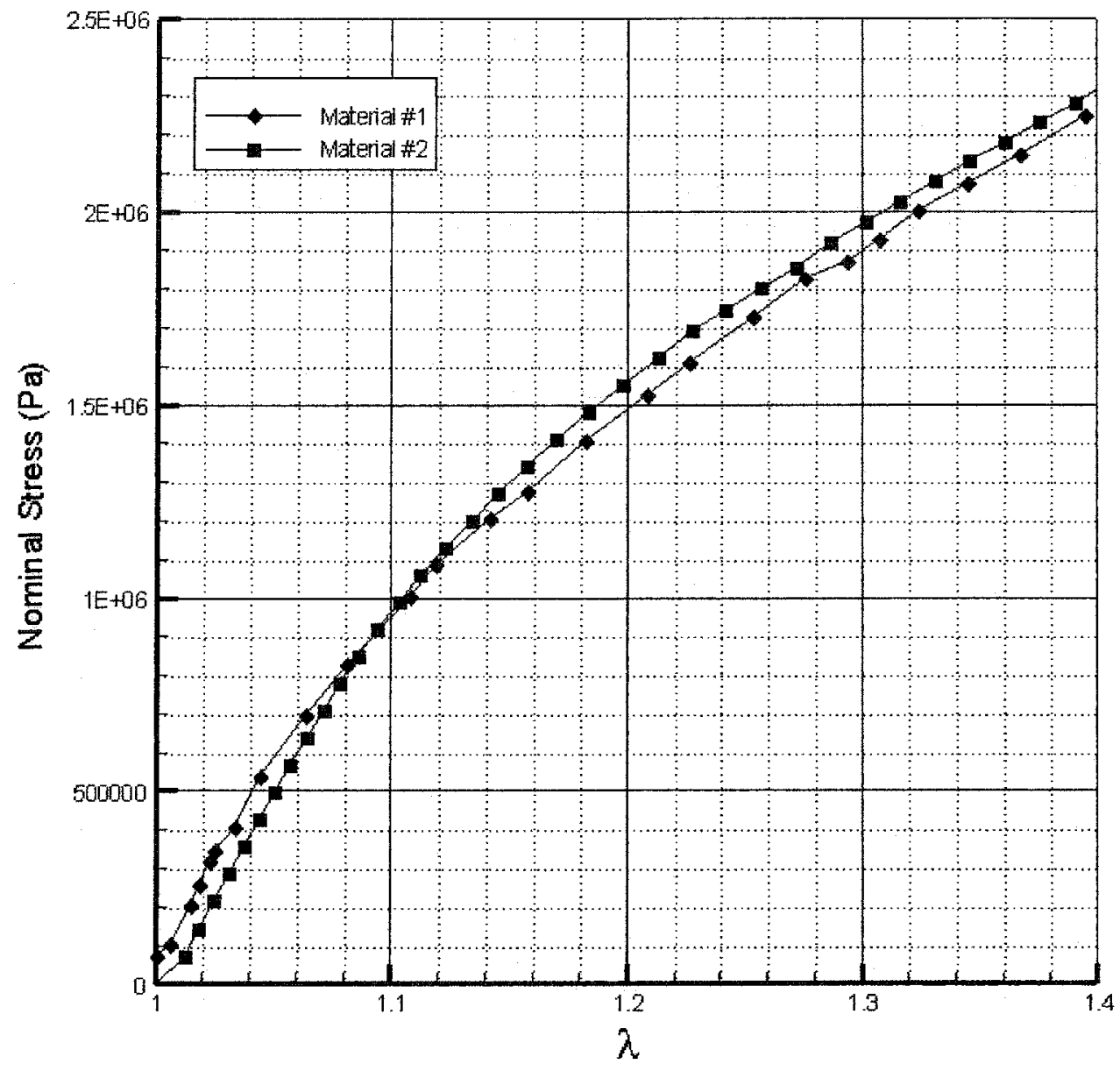

Figure 4.8: Uniaxial tensile data for Material \#1 and Material \#2 (Biomer clones) 


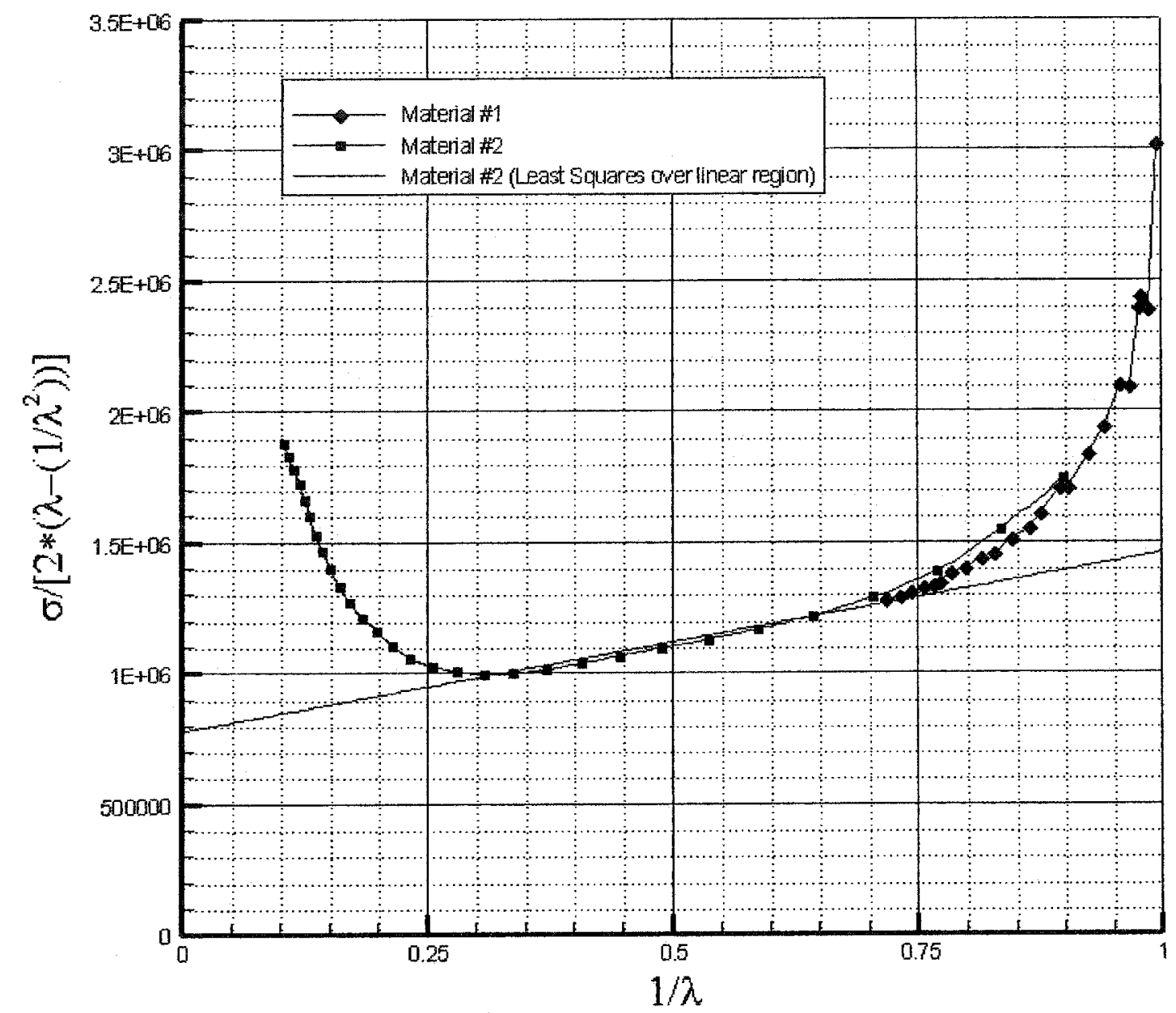

Figure 4.9: Mooney-Rivlin Plot of Uniaxial Tension

tension form of the Mooney-Rivlin Equation 4.32. The Mooney-Rivlin coefficients can be determined by rearranging Equation 4.32 and plotting, $\frac{\sigma_{N}}{2\left(\lambda-\frac{1}{\lambda^{2}}\right)}$ versus $\frac{1}{\lambda}$. Note here that $\sigma_{N}$ is the nominal stress as the data collected in terms of nominal stress. Thus, the uniaxial data can be represented as a straight line with the y-intercept as the $C_{10}$ coefficient and the slope of the line as $C_{01}$.

The Mooney-Rivlin plot for uniaxial tension is shown in Figure 4.9. It is important to note the Mooney-Rivlin equation in terms of stretch variables as shown in Equation 4.32 is highly non-linear. When stress-strain data is transformed into the Mooney-Rivlin plot 
as shown in Figure 4.9, due to the reduced strain $\frac{1}{\lambda}$, the data appears to be plotted in the reverse direction. For example, as shown in Figure 4.8, data points plotted from $0 \%$ to $40 \%$ are plotted from left to right, and in the Mooney-Rivlin plot those same data points are plotted from right to left, or within 1-0.7, as shown in Figure 4.9.

It is desirable that a linear region appears in the transformation of the original data to the Mooney-Rivlin plot. However, for many engineering elastomeric materials, such as carbon black rubber, the transformation generally gives a $\mathrm{U}$-shaped plot as shown in Figure 4.9. Thus, to obtain a straight line, the range of data at the bottom of this $U$ shaped curve, normally appearing linear, is typically selected for analysis. A least squares method is used to produce a linear curve from which the Mooney-Rivlin coefficients can be determined. This is accomplished in Excel by the use of a linear regression option when graphing the data.

In Figure 4.9 the region 1-0.9, (or, in the original data, between 0 and $10 \%$ strain), shows the undesired non-linear 'u-shaped' result of the transformation. It is important to note that it is the highly non-linear nature of the Mooney-Rivlin equation that creates the instability at the 1-0.9 range in the Mooney-Rivlin plot as seen clearly in Figure 4.9. Thus, the range from 1-0.9 in the Mooney-Rivlin plot is generally ignored.

It is important to state that the accuracy of the linear fit is good only over the selected range of strain as indicated by Gent [38]. Indeed, when selecting the region to fit the data on the Mooney-Rivlin plot, Figure 4.9, the resulting coefficients are only good for that range on the original stress-strain plot, Figure 4.8. Thus, various ranges of stretch were selected to determine the best fit for the strain range, $0-40 \%$, on the stress-strain plot, Figure 4.8 .

The diaphragm is expected to see strains of at least $50 \%$. On the Mooney-Rivlin plot this corresponds to a range of 1-0.7. In this range both curves increase in slope rapidly as shown in Figure 4.9. It was determined that the best fit for uniaxial data in the strain 


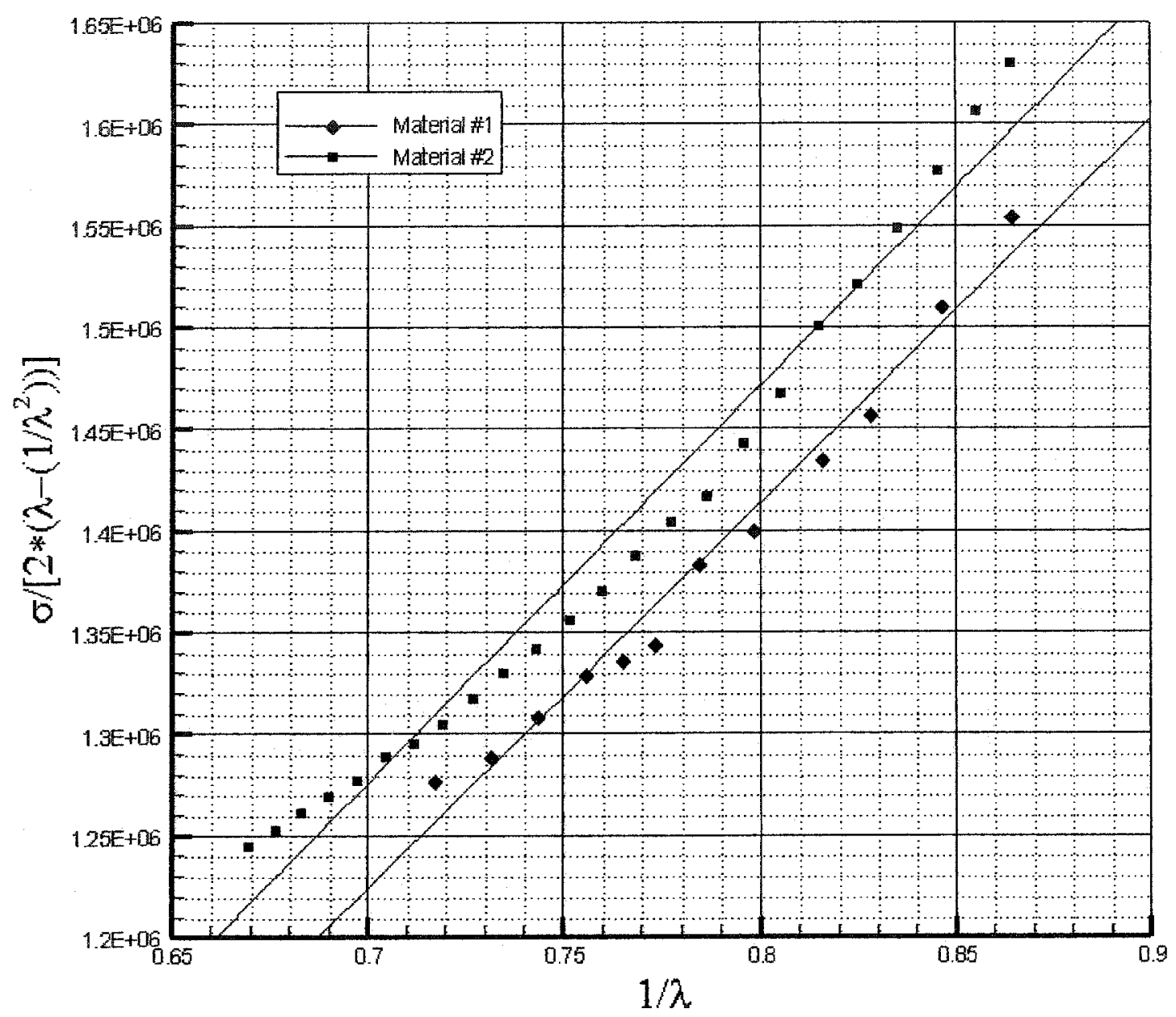

Figure 4.10: Mooney-Rivlin Plot over range of interest (ROI)

range of $0-40 \%$ occurred with a least squares fit on the Mooney-Rivlin plot through the range 0.9-0.7 as shown in Figure 4.10.

ABAQUS accepts user input stress-strain data points and generates its own MooneyRivlin coefficients based on a least squares method. It is recommended that data points in strain range of most interest should be entered. The high strain points may force the fitting to lose accuracy and/or stability in the low strain range. It is expected that due to the relatively high stiffness of polyurethane, the membrane strains will be very small or at least less than $40 \%$. Also it is recommended that the data points are entered at evenly spaced 


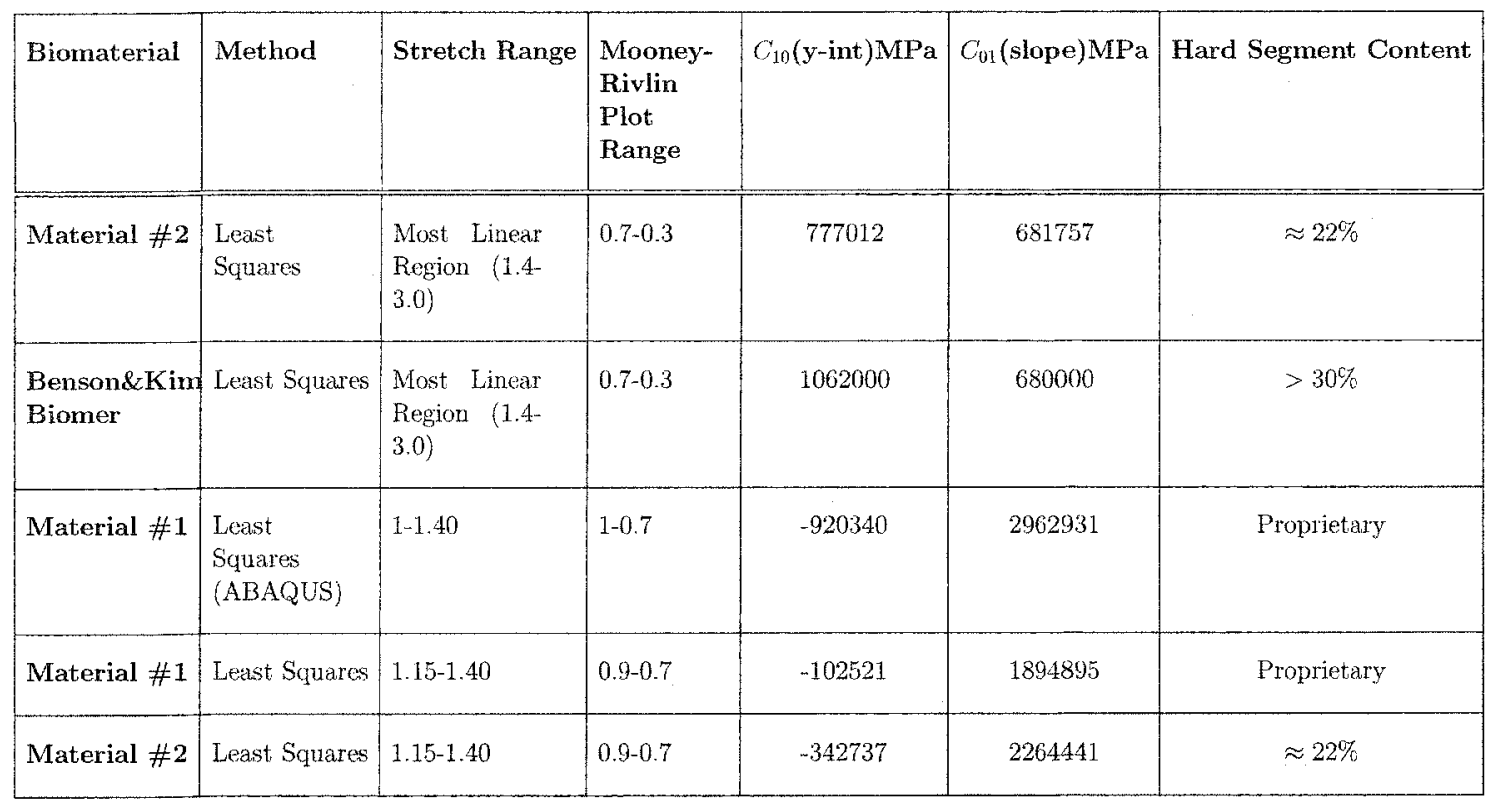

Table 4.1: Mooney-Rivlin coefficients for Biomer clones and published Biomer data for strains in uniaxial tension

strain intervals over the range of interest to allow similar accuracy throughout the entire strain range. The data provided for Material \#1, 0-40\% strain was all entered into the ABAQUS software. ABAQUS also applied a least squares fit to the data. Mooney-Rivlin coefficients determined over various ranges are shown in Table 4.1 for comparison.

The physical meaning of the Mooney-Rivlin coefficients presented in Table 4.1, begins with an explanation of the first two rows. Row 1 in Table 4.1 shows the Mooney-Rivlin coefficients for Material \#2 determined from the most linear region, shown clearly in Figure 4.9. Row 2 presents the coefficients published by Benson and Kim [12]. Their analysis was also over the most linear region of the Mooney-Rivlin plot in uniaxial tension. The $C_{01}$ coefficients are the same over the linear region of the curve. The $C_{10}$ coefficients are different. As was mentioned earlier, in Chapter $3, C_{10}$ and $C_{01}$ have physical meaning. $C_{10}$ represents the crosslink density of the polyurethane. However, the crosslink density is not the same as in the traditional sense of sulphur crosslinks. Polyurethanes soft segments are held together by hard segment aggregates, as shown in Figure 3.5. Hence, the crosslink 
density is related to the amount of hard segment. Material \#2 is reported to have a hard segment content of approximately $20 \%$ and although Biomer composition is proprietary, it is expected that is has a higher crosslink density, $>30 \%$, as noted also by Benson and Kim [12]. Therefore, it is expected that a higher $C_{10}$ value is observed for Biomer if the same linear region is analyzed on the Mooney-Rivlin plot.

Row 3 of Table 4.1, shows the coefficients from ABAQUS least squares fit over all data provided for Material \#1, 0-40\%. As can be seen from Figure 4.9, fitting the curve over all the data is not suitable because, as mentioned earlier, the region from 1-0.9 on the Mooney-Rivlin plot is highly unstable and this instability is a result of the sensitive, nonlinear Mooney-Rivlin equation in terms of stretch, Equation 4.32. Thus, this information is shown only as confirmation that ABAQUS does use similar methods to the least squares fit method that is used by the author.

Row 4 and row 5 show the coefficients for the range of best fit for the stress-strain curve for Material \#1 and Material \#2. However, it is noted that the values of $C_{10}$ are negative and the $C_{01}$ term, the slope of the line, has increased significantly. This may be explained, once again, by the morphology of the polyurethane. In the region of strain $<50 \%$, it is known that a different phenomenon is occurring [39] [12]. The degree of attachment of polyurethanes at low strains depends mostly on hard and soft phase interaction. It was suggested by Abraham et al. [39] that the low hysteresis behaviour, in the low strain range, $<50 \%$, is due to the ability of the dispersed hard segments to maintain phase interaction with the soft segment sa load is applied. Thus, the secondary forces of hydrogen bonds and van der Waals bonds between the hard and soft segment chains creates a high level of energy dissipation.

If a reduction in the $C_{01}$ coefficient, slope, is observed as strain increases, it is suggested that this is due to the reduction of the secondary bond interaction between the hard and soft segments, i.e. hydrogen or van der Waals forces. Hence, as strain increases, the value 
of $C_{01}$ diminishes as the slope of the Mooney-Rivlin plot decreases.

It is also reasonable to see smaller values $C_{10}$ in this range. The hard segment appear to behave more like standard network crosslinks, such as sulphur crosslinks, only when there is a significant amount of stretching occurring in the specimen, such as values $>50 \%$.

Thus, this uniaxial data analysis reveals that there are two distinct regions of the Mooney-Rivlin plot for polyurethane materials in uniaxial tension, $<50 \%$ and $>50 \%$. And hence, the selected range for least squares analysis, is taken from 0.9-0.7. Isolating this range of interest ensures a more accurate fit of the original uniaxial data to the coefficients obtained from the least squares fit over this range. From the above discussion, it also appears to better represent the increasing effect of phase interaction between hard and soft segments at the low strain regions that is characteristic of polyurethane thermoplastic elastomers. The morphological explanation above explains why $C_{01}$ values are higher and the $C_{10}$ are lower at the low strain regions. The values are shown as such in Table 4.1 Rows 4 and 5 .

In summary, the Mooney-Rivlin equation is capable of describing the uniaxial stressstrain response accurately for carbon black filled rubber with standard sulphur crosslinks up to strains of $200 \%$. One set of coefficients is obtained from data in the linear range of the Mooney-Rivlin plot and one Mooney-Rivlin equation is required for black rubber.

The Mooney-Rivlin plot for polyurethane elastomers is non-linear, however, it does seem to represent polyurethane materials well enough, in uniaxial tension, if the plot is isolated in 2 separate regions, $<50 \%$ and $>50 \%$, ie. before and after significant phase separation of the hard and soft segments.

The coefficients can be evaluated for their accuracy by creating stress-strain curves using the coefficients from Rows 1, 2 and 3 in Table 4.1 and the same Mooney-Rivlin Equation 4.32. and are shown in Figure 4.11.

Superimposed on Figure 4.11, are the original stress-strain curves shown in Figure 4.8. 


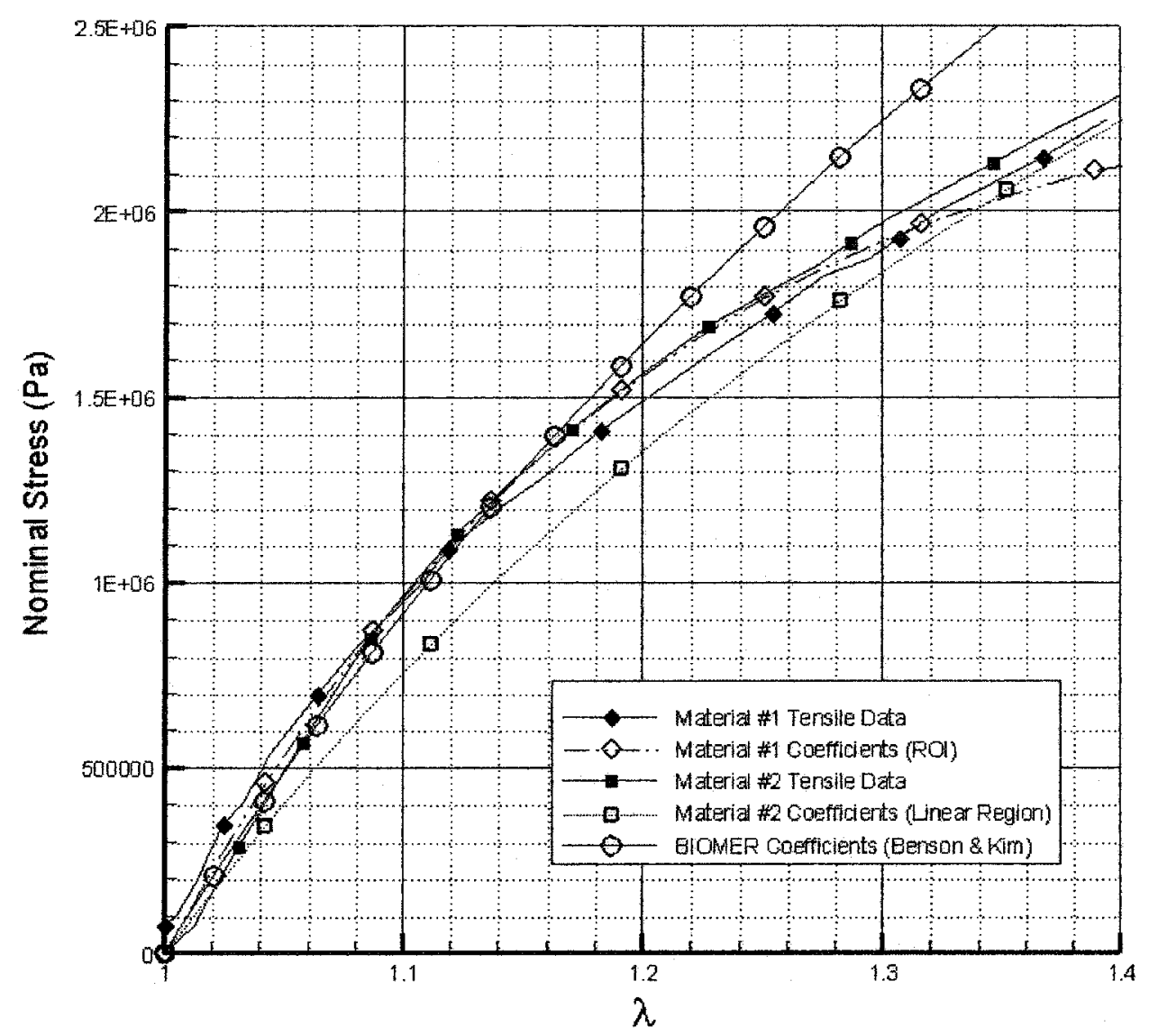

Figure 4.11: Evaluation of Mooney-Rivlin coefficients shown in Table 4.1 
The results show that if coefficients using the most linear region of the Material \#2 plot, Row 1 , are evaluated, the response is underestimated by as much as $0.25 \mathrm{MPa}$ or $25 \%$ of the stress, at $18 \%$ strain. The ABAQUS coefficient evaluation shows good correlation with the original stress-strain curves up to $35 \%$ strain, even though the fit was calculated using data in the region of instability, 1-0.9. Nevertheless, after this point, there is a marked decrease in the curve. The response of the Biomer coefficients shows a stiffer response when compared against the full Material \#2 curve shown in the inset, and this is in agreement with the higher concentration of hard segments contributing to the crosslink density mentioned earlier in the section.

\subsubsection{Biaxial Tensile Test}

Biaxial tensile data was not readily available Material \#1. Furthermore, biaxial data for Biomer in the virgin state was also difficult to obtain. Therefore, it was decided to carry out limited biaxial tests on diaphragms, manufactured from the Material \#1. The test chosen, for simplicity, was an inflation test. Fortunately, the biaxial test apparatus was built after the samples arrived and the test base and clamp was designed accordingly, to fit the flat region of the cast diaphragm. This test, the apparatus, procedure and data collection, as well as the reduction of data including the calculation of the Mooney-Rivlin coefficients is described in the following sections.

The Mooney-Rivlin coefficients for biaxial extension can be determined by rearranging Equation 4.33 and plotting, $\frac{\sigma_{22,11}}{2\left(\lambda^{2}-\frac{1}{\lambda^{4}}\right)}$ versus $\lambda^{2}$. Notice here that the true stress is used to determine the coefficients as opposed to the nominal stress, as in the previous section. Thus, the uniaxial data can be represented as a straight line with the y-intercept as the $C_{10}$ coefficient and the slope of the line as $C_{01}$. The linear regression fit can once again be determined using the least squares method. 


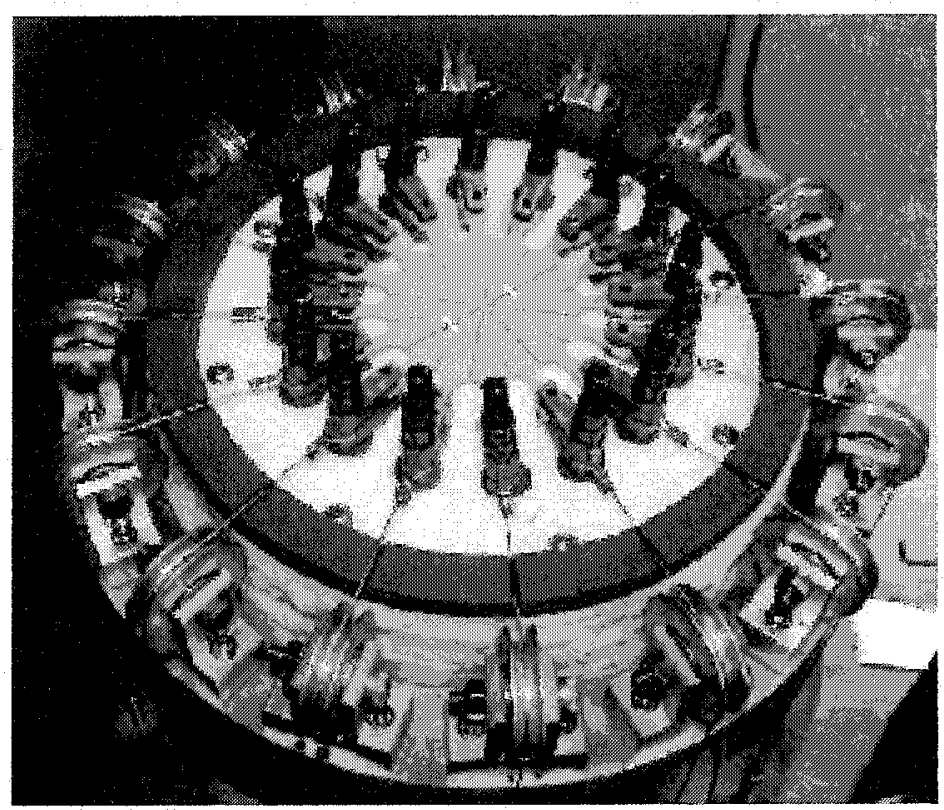

Figure 4.12: AXEL apparatus to determine biaxial constants [45]

\section{Inflation Test}

To determine biaxial stretch ratios, an inflation test was used. Essentially, the test consists of clamping a small circular sheet of the material and subjecting it incrementally to internal pressure and recording the resulting stretch at the pole of the inflated shape. If the measurement locations for stretch are at the pole of the inflation or center of the disk of material, then the curvature of the material can be ignored and the local region is assumed to be under biaxial load. The method was used by Treloar [44], to find material constants and also check the validity of analytical solutions for membrane inflation. The inflation test is simpler to implement than for example, stretching rubber in two orthogonal directions by means of an actuated strain machine. One method to determine hyperelastic constants using a specialized apparatus is shown in Figure 4.12 


\section{Apparatus}

The inflation test apparatus was machined at the Carleton University Mechanical and Aerospace Machine Shop. It consists of a 1 inch thick circular 6061 aluminum base with an air inlet and pressure tap. A 0-180 PSI pressure gauge is screwed into the base and the air inlet is connected with a regulator. A cavity in the base allows dry compressed air to pressurize the rubber. A machined annular 6061 aluminum clamp is placed over a specimen and screwed down to the base by means of 5 flat head hex screws radially located around the clamp. The Material \#1 specimen has grid lines drawn on its surface so that the deformation can be measured during inflation.

The apparatus is placed on a Nikon optical comparator which can measure deformation in 3 principal directions as shown in Figure 4.14. The base and clamp with the mounted specimen, the attached pressurization inlet, hose and pressure transducer, is clamped to the $\mathrm{x}-\mathrm{y}$ stage, and positioned directly under the optical comparator viewing lens. Using precision $\mathrm{x}-\mathrm{y}$ screw micrometers attached to the $\mathrm{x}-\mathrm{y}$ stage, the entire apparatus can be horizontally positioned under the lens. The entire stage is attached to a rack and pinion jack that allows for precision vertical translation of the $\mathrm{x}-\mathrm{y}$ stage. A $10 \mathrm{x}$ magnification lens projects the specimen grid lines through the lens and on to a viewing screen where the user can judge the focus. A dial gauge mounted adjacent to the metal base of the optical comparator, probes the vertical location of the $\mathrm{x}-\mathrm{y}$ stage and thus the $\mathrm{z}$-axis translation of the bubble inflation.

Test specimens from finished components were available for Material \#1. Ten samples were provided and six were used to evaluate the material. The cast diaphragm shape in its supplied form is curved at the edges but contains a uniform flat circular surface of adequate radius for mounting and inflation. Each specimen was cut to fit the free inflation zone of 1.1755 inches in diameter and to ensure the entire periphery of the specimen is sufficiently clamped. 


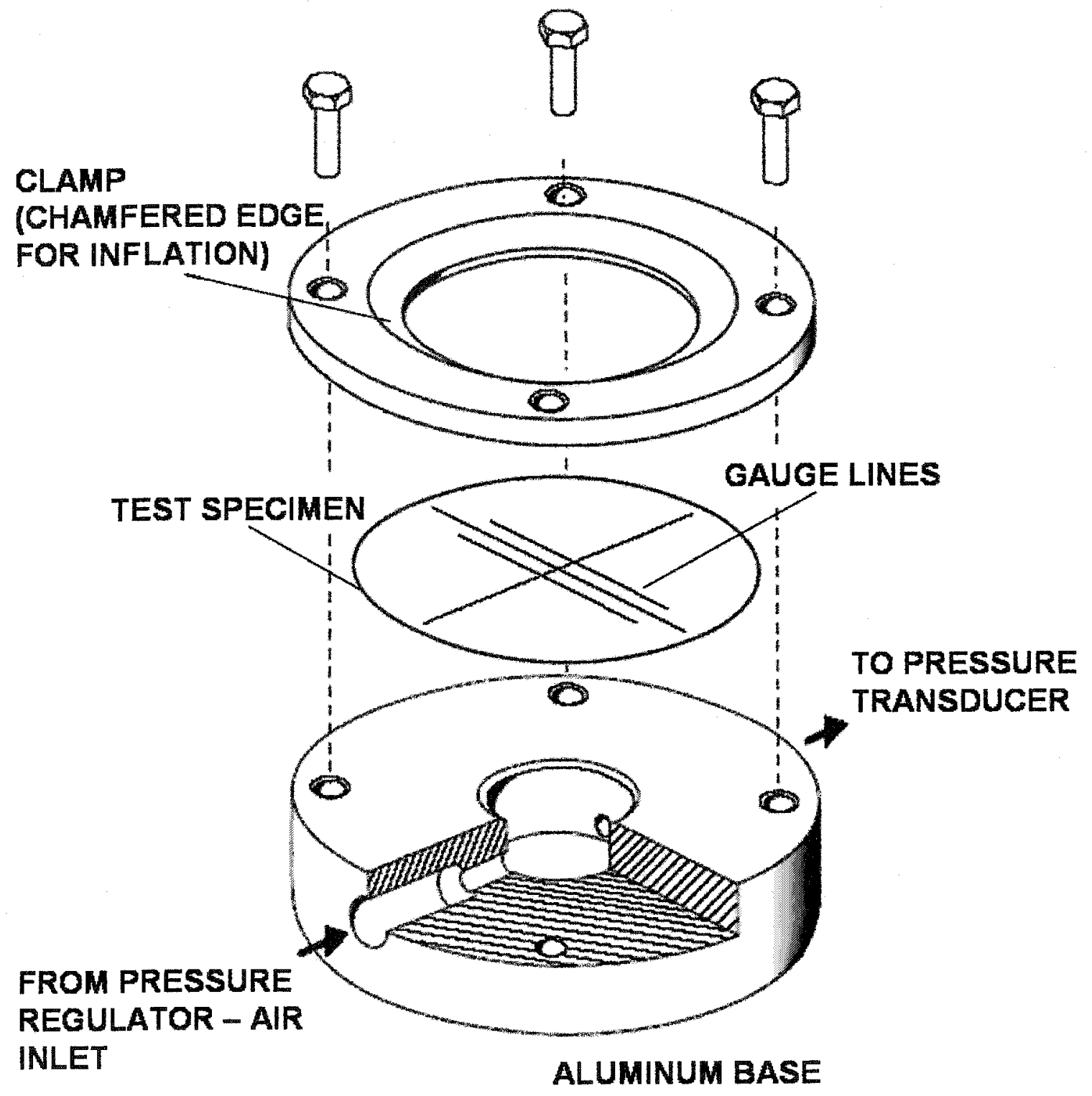

Figure 4.13: Exploded view of base and clamp [46] 


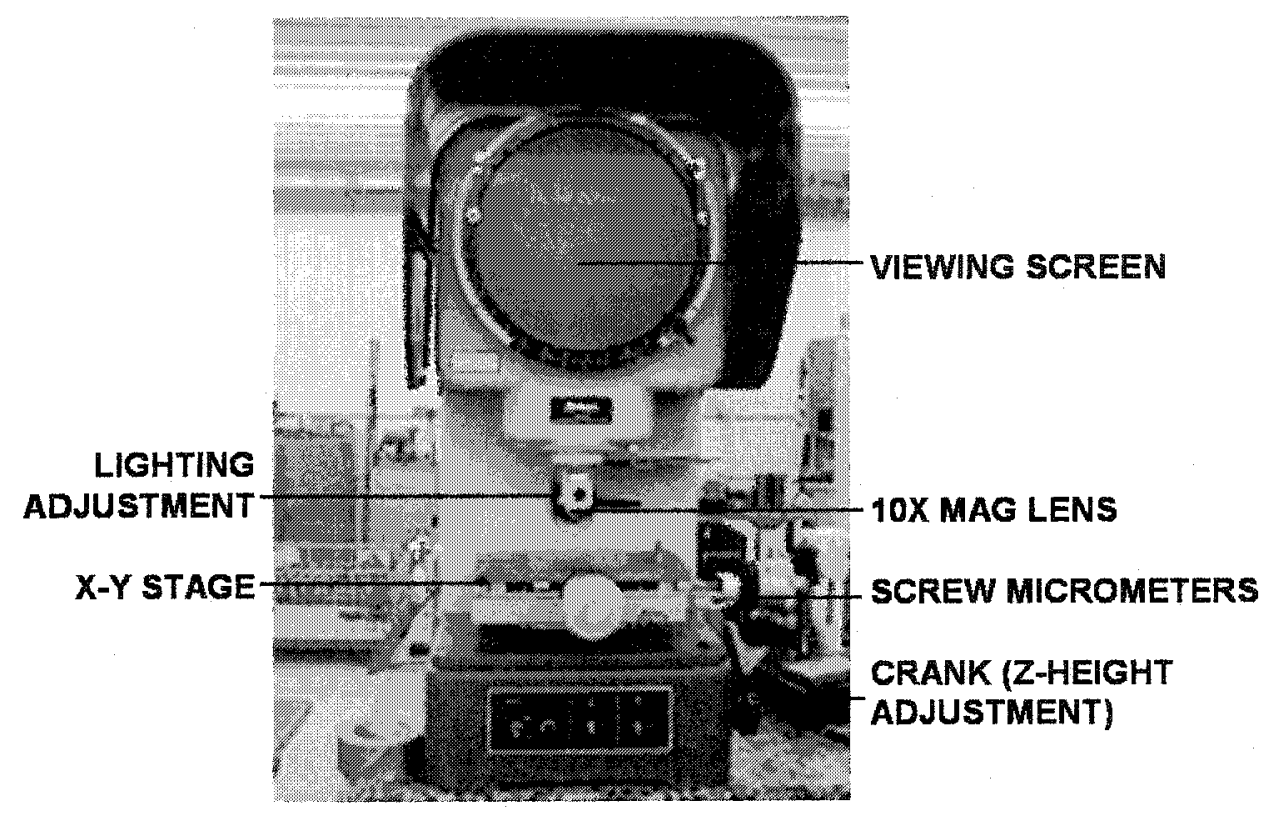

Figure 4.14: Nikon Optical Comparator

It is important to ensure the clamping force is sufficient to avoid leaking as well as slippage of the sample during each successive pressure state as noted by [46]. However, in practice, too high a clamping force causes the sample to deform and may cause prestress in the material. A judgement must be made as to how tight the screws should be. To reduce the slippage of the specimen during inflation, the surfaces of the clamp and the base are lightly etched with 500 grit sandpaper. The zone of etching on both surfaces is in a specific annular ring location. After etching with 500 grit paper, 200 grit sand paper was used to deburr any large pieces that may tear the specimen during inflation.

The stretch ratio that is to be determined is given by:

$$
\lambda=\frac{l_{s}}{l_{o}}
$$

where $l_{s}$ is the stretched gauge length and $l_{0}$ is the original length as shown in Figure 4.15. The $l_{s}$ cannot be determined directly and hence we use the optical comparator 


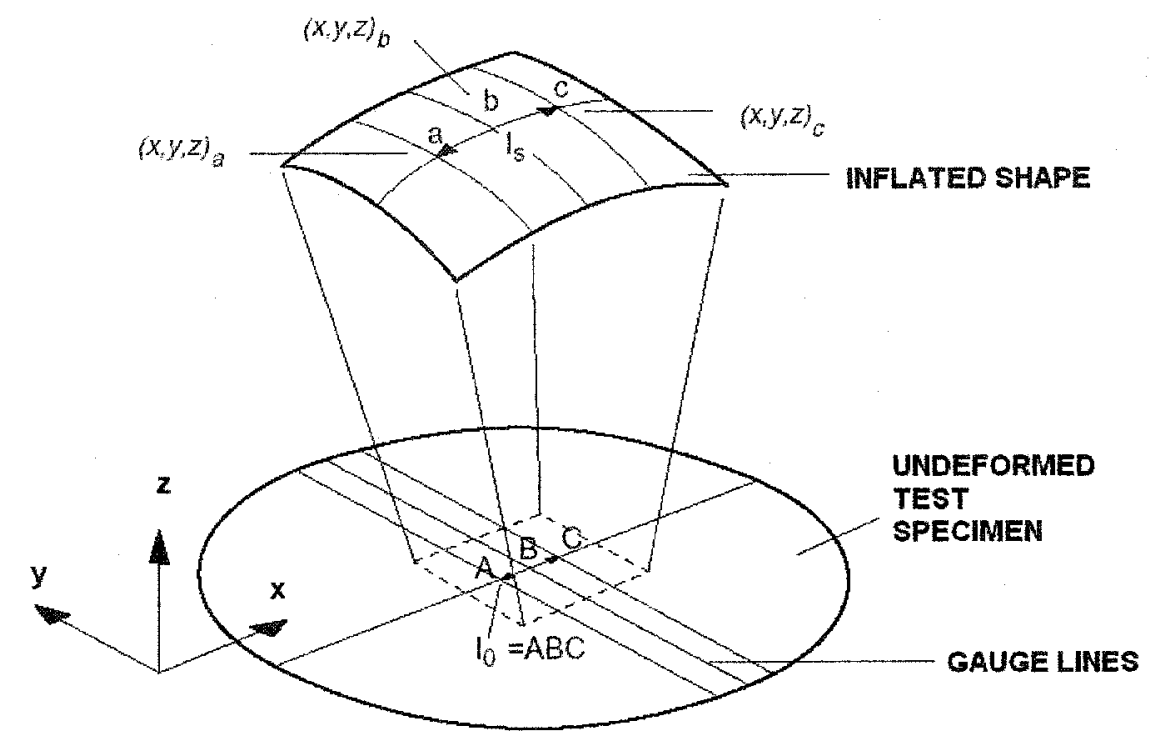

Figure 4.15: Equidistant point measurement [46]

to determine the location of the marker points $a, b$, and $c$ of the gauge lines in 3D space as shown in Figure 4.15.

The centre marker shown in Figure 4.15 as $(x, y, x)_{b}$ does not necessarily have to be at the absolute apex of the inflated membrane.

To create the measurement points, 3 evenly spaced lines were drawn using a waterbased black ink for the translucent Material \#1 material. Gauge lines were drawn at the centre of the sample at equidistant points less than $10 \mathrm{~mm}$ apart. A straight edge was used to draw a perpendicular line diametrically across the 3 lines. The 3 points can then be taken as intersection of the lines.

Ink adhesion to the surface was not a concern as $\lambda$ is less than 2 and it is still possible to track the points along the pole without the locations blurring. In practice, it was not necessary to accurately mark the gauge lines as long as the original material point was tracked through each successive pressurization.

The test procedure is as follows: 
1. Focus the projected image in the viewing screen on the specimen surface using the marker gauge lines.

2. Rotate the screw micrometers to the position of the desired point below the crosshairs of the optical comparator viewing screen.

3. Refocus by translating the $x-y$ stage using the crank on the side of the comparator.

4. Realign the crosshairs if focussing improves the image.

5. Manually record the value given by the vernier scale and the dial gauge.

6. Repeat step 2-3 for each of the other 2 points.

7. Admit air into the apparatus.

8. Allow 2-3 minutes to allow the pressure source to reach equilibrium. Measure the pressure.

9. Repeat steps $2-7$ until the maximum pressure is reached.

\section{Sources of Error and Limitations of the Apparatus}

Before proceeding with calculation of the coefficients from the raw data it is necessary to state the precision of the instruments used and to state the accuracy of the data.

Variation in the data may be attributed to a variety of sources. Firstly, testing rubber samples cut from final components is often done, however, tensile strength and elongation at break values are said to be $10 \%$ lower for rubber materials [38]. Secondly, test samples can vary depending on the batch it was prepared from, and thus, final component test pieces may also carry this variation. Finally it is recommended that materials be cycled 320 times to account for hysteresis in the stress-strain behaviour of rubber [38]. This stress relaxation is called the Mullins effect and removes potentially higher stresses obtained from virgin material characterization. Given that this thesis is a first approach this cycling was not conducted. However, it is important to note that hysteresis effect is relatively low in the strain region of interest in this study $<50 \%$ as shown in Figure 4.5 . 


\begin{tabular}{|l|l|}
\hline Instrument & Precision \\
\hline Dial Gauge & $1.3 \times 10^{-5}$ metres \\
Screw Micrometers & $25.4 \times 10^{-5}$ metres \\
Pressure Transducer & $\pm 2757.9 \mathrm{~Pa}$ \\
\hline
\end{tabular}

Table 4.2: Precision of instruments for inflation test

The pressure transducer is another source of potential error as the precision corresponds to $\pm 23 \mathrm{mmHg}$. The error in stretch can be as much as $\pm 10 \%$ at $5 \%$ strain. Due to the small thickness, the hoop stress relation amplifies this error. Despite the limitations of the apparatus, consistent results were obtained. The pressure versus strain curve is shown in Figure 4.16.

It is important to note that the strain range tested, as shown in Figure 4.16, was between $0-25 \%$ due to the limitations of the apparatus. The pressure regulator controlling the air pressure behind the inflated membrane has a range of 0-20 PSI and the dial gauge and $\mathrm{x}-\mathrm{y}$ stage have a maximum range of 1 inch. This resulted in a limitation of the data to the strain range observed.

The stretch that is shown in Figure 4.16 , is calculated by means of trigonometric relationship that is described in the next section.

\subsubsection{Calculation of Mooney-Rivlin Coefficients}

The deflection data collected must be reduced into a form from which the coefficients of Equation 4.33 can be extracted.

The deformed gauge length $l_{s}$ can be determined from the radius of curvature $r$, obtained by a trigonometric relationship. With the deformed gauge length, the stretch ratio, $\lambda$, can be determined. With the original and deformed gauge lengths coplanar, the radius of curvature can be determined by the following trigonometric relationship with the variables defined in Figure 4.17. 


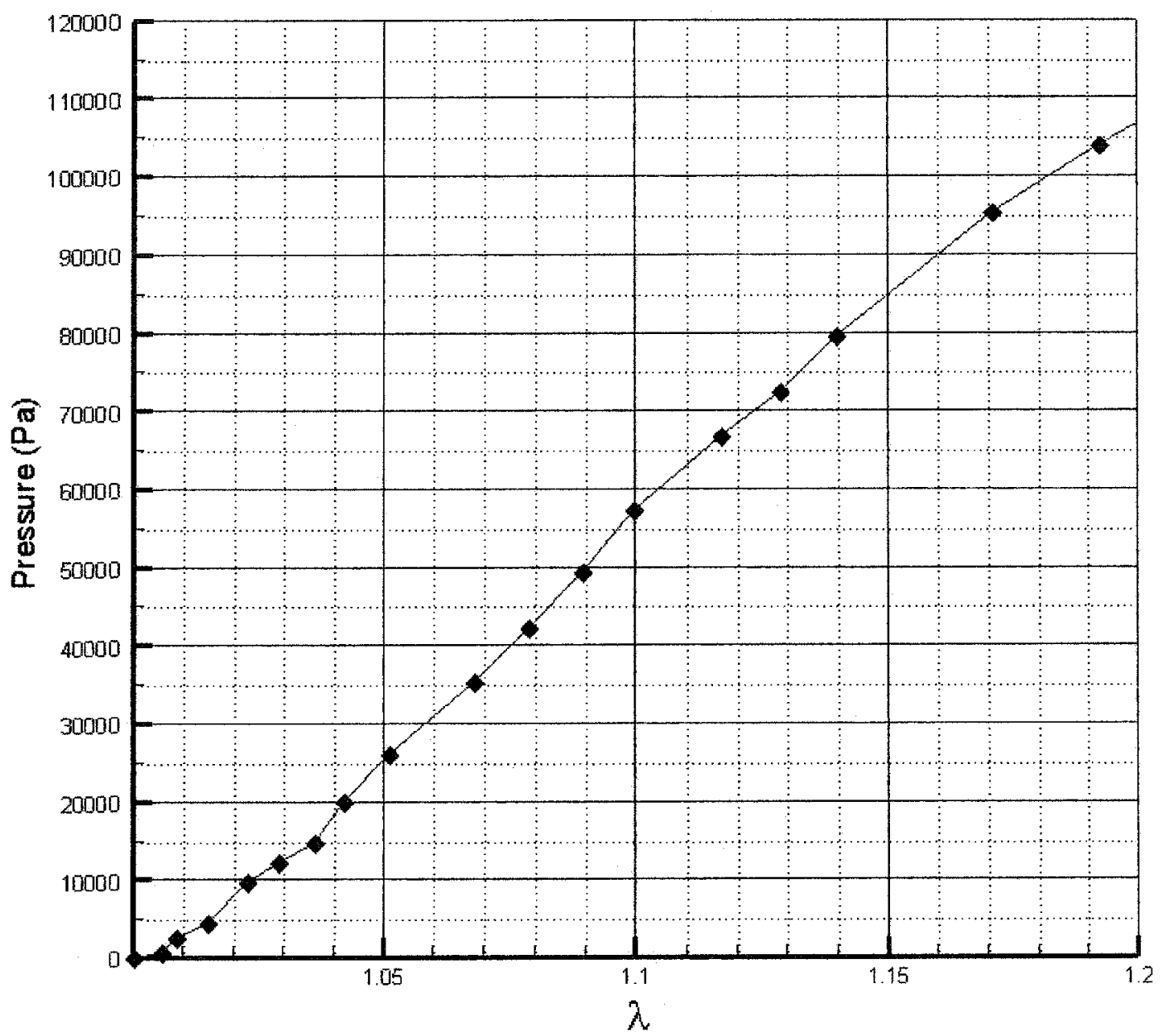

Figure 4.16: Inflation pressure-stretch curve for Material \#1 


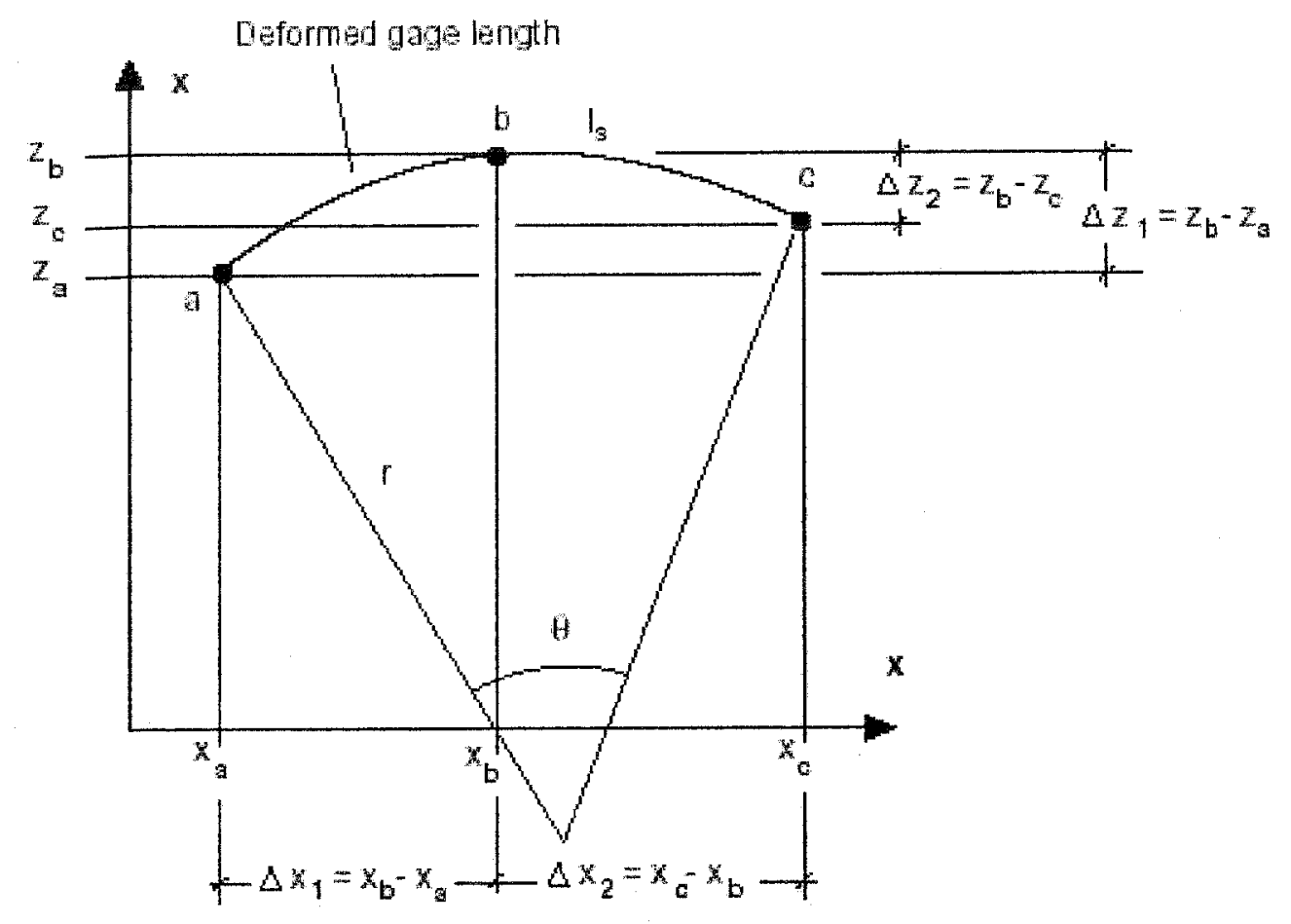

Figure 4.17: Variable definition for trigonometric relationship to determine radius of curvature [46]

$$
r^{2}=\frac{1}{4} \frac{\left(\Delta x_{1}^{2}+\triangle z_{1}^{2}\right)\left(\Delta x_{2}^{2}+\triangle z_{2}^{2}\right)\left(\left(\triangle x_{1}+\triangle x_{2}\right)^{2}+\left(\triangle z_{1}-\triangle z_{2}\right)^{2}\right)}{\left(\triangle x_{1} \triangle z_{2}+\triangle x_{2} \Delta z_{1}\right)^{2}}
$$

The above approach was used by Makino et al. [46] and verified using a CAD software called GID Ver. 7.2. The angle subtended by the deformed gauge length, $\theta$ can be also be determined by:

$$
\theta=2 \arcsin \frac{1}{2 r} \sqrt{\left(\triangle x_{1}+\Delta x_{2}\right)^{2}+\left(\triangle z_{1}-\Delta z_{2}\right)^{2}}
$$

The deformed gauge length can be found from the arc length:

$$
l_{s}=r \theta
$$


as the radius of curvature is much greater than the length. Figure 4.17 is an exaggerated diagram for explanation purposes.

The Cauchy stress in the biaxial state near the pole of the inflated shape is found using the hoop stress:

$$
\sigma_{11}=\sigma_{22}=\frac{p r}{2 t_{s}}
$$

where $\sigma_{11}, \sigma_{22}$ are the stresses, $p$ is the inflation pressure, $r$ is the radius of the free zone of inflation and $t_{s}$ is the thickness of the sheet in the deformed state. From the equibiaxial constraints shown in Figure 4.2 , the deformed thickness, $t_{s}$ is actually a function of the original thickness, $t_{o}$, and the stretch ratio:

$$
\lambda_{3}=\frac{1}{\lambda^{2}}=\frac{t_{s}}{t_{o}}
$$

Equation 4.40 is used in Equation 4.39, the stress is then:

$$
\sigma_{11}=\sigma_{22}=\frac{p r}{2 t_{o}} \lambda^{2}
$$

An Excel spreadsheet is used to determine the true stress and true stretch values and are plotted in Figure 4.18. Superimposed on this graph is the uniaxial curve for comparison. Biaxial stresses are higher than the uniaxial stress for the same strain.

The Mooney-Rivlin plot for biaxial tension is shown in Figure 4.19. The MooneyRivlin equation for biaxial stretch ratios, produces a different curve than the uniaxial case. The $\mathrm{x}$-values are no longer $\frac{1}{\lambda}$ but are now $\lambda^{2}$. The transformation to Mooney-Rivlin plot for biaxial tension, shows instability in the region from 1-1.15 on the $\mathrm{x}$-axis of the Mooney-Rivlin plot corresponding to 0 to $10 \%$ strain in the original biaxial stress-strain data. Figure 4.19, shows undesired perturbations in the lower strain region.

The coefficients obtained from the least squares method on the Mooney-Rivlin plot is 


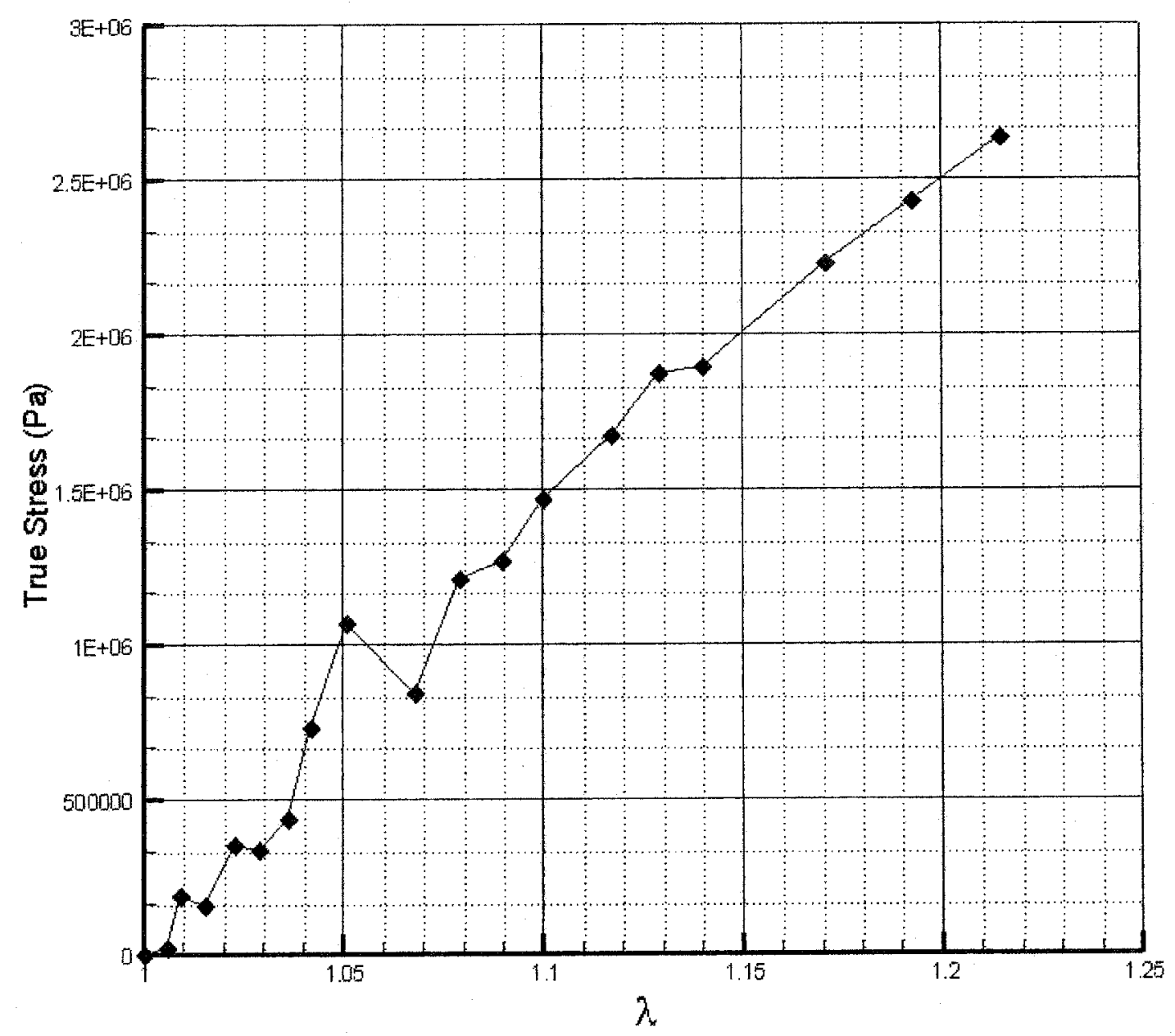

Figure 4.18: Biaxial stress-stretch curve for Material \#1 


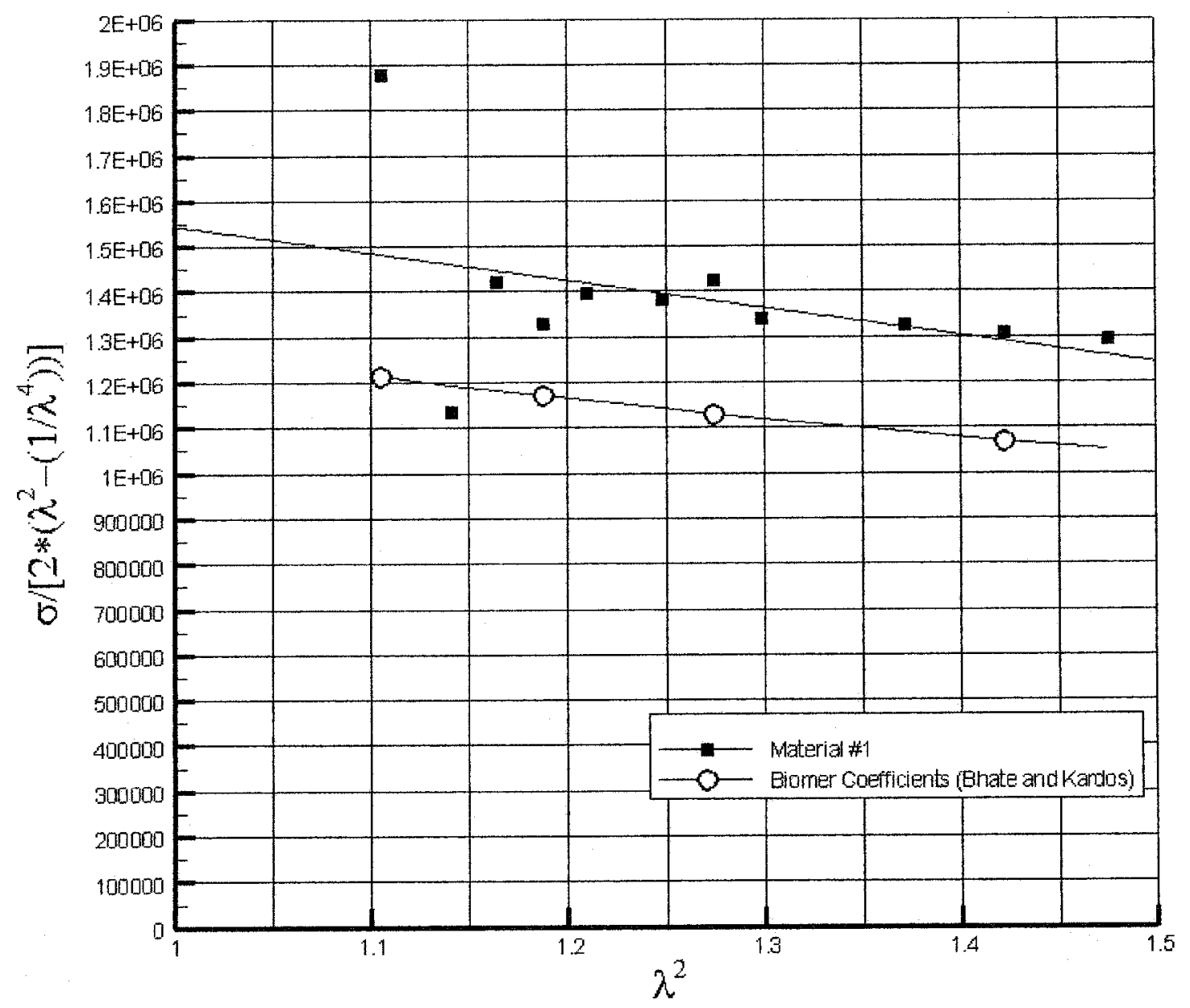

Figure 4.19: Mooney-Rivlin plot for the biaxial strain case for Material \#1 


\begin{tabular}{|c|c|c|c|c|c|}
\hline Biomaterial & Method & Strain Range & $\begin{array}{l}\text { Mooney- } \\
\text { Rivlin Plot } \\
\text { Range }\end{array}$ & $\mathrm{C}_{10}(y$-int $)(\mathrm{MPa})$ & $\mathrm{C}_{01}($ slope $)(\mathrm{MPa})$ \\
\hline Material \#1 & Least Squares & Most Linear Region $(5-22 \%)$ & $1.1-1.48$ & 2149455 & -604021 \\
\hline
\end{tabular}

Table 4.3: Mooney-Rivlin coefficients for Material \#1 and published values for Biomer for biaxial tension

compared against published data from Bhate and Kardos [34] and shown in Table 4.3.

It is proposed that the coefficients obtained from applying the Mooney-Rivlin equation to biaxial strain has physical significance. Should those same coefficient values be used on the uniaxial plot, in Figure 4.9, a linear curve with a decreasing slope would correspond the left end of the uniaxial Mooney-Rivlin plot. This region on the uniaxial curve corresponds to soft segment crystallization as shown in Figure 3.5. This suggests a different physical change is occurring in biaxial tension at low strains, in the polymer network, compared to the uniaxial tensile case at low strains. In the biaxial case, it is possible as strain is being induced in all directions, the phase separation does not occur as rapidly as in the uniaxial case where hard segments realign in the direction of strain [39]. This suggests that dispersed hard segments act more as crosslinks in the network at low strains and this corresponds to high $C_{10}$ value as shown in Table 4.3. In other words, the biaxial stressstrain curve is stiffer because of the nature of straining. It is possible that this phenomenon also occurs in the uniaxial case in the high strain range, (low values of Mooney-Rivlin plot) where the $\mathrm{U}$ shaped curve turns upwards again, and the morphology is as shown in Figure 3.5.

The coefficients shown in Table 4.3 can be evaluated against the original data by simply substituting the coefficients into Equation 4.33 and plotting stress versus stretch as shown in shown in Figure 4.20. The Biomer data from Bhate and Kardos study, appears more compliant than our findings. The biaxial data from that test was taken using a tuned 


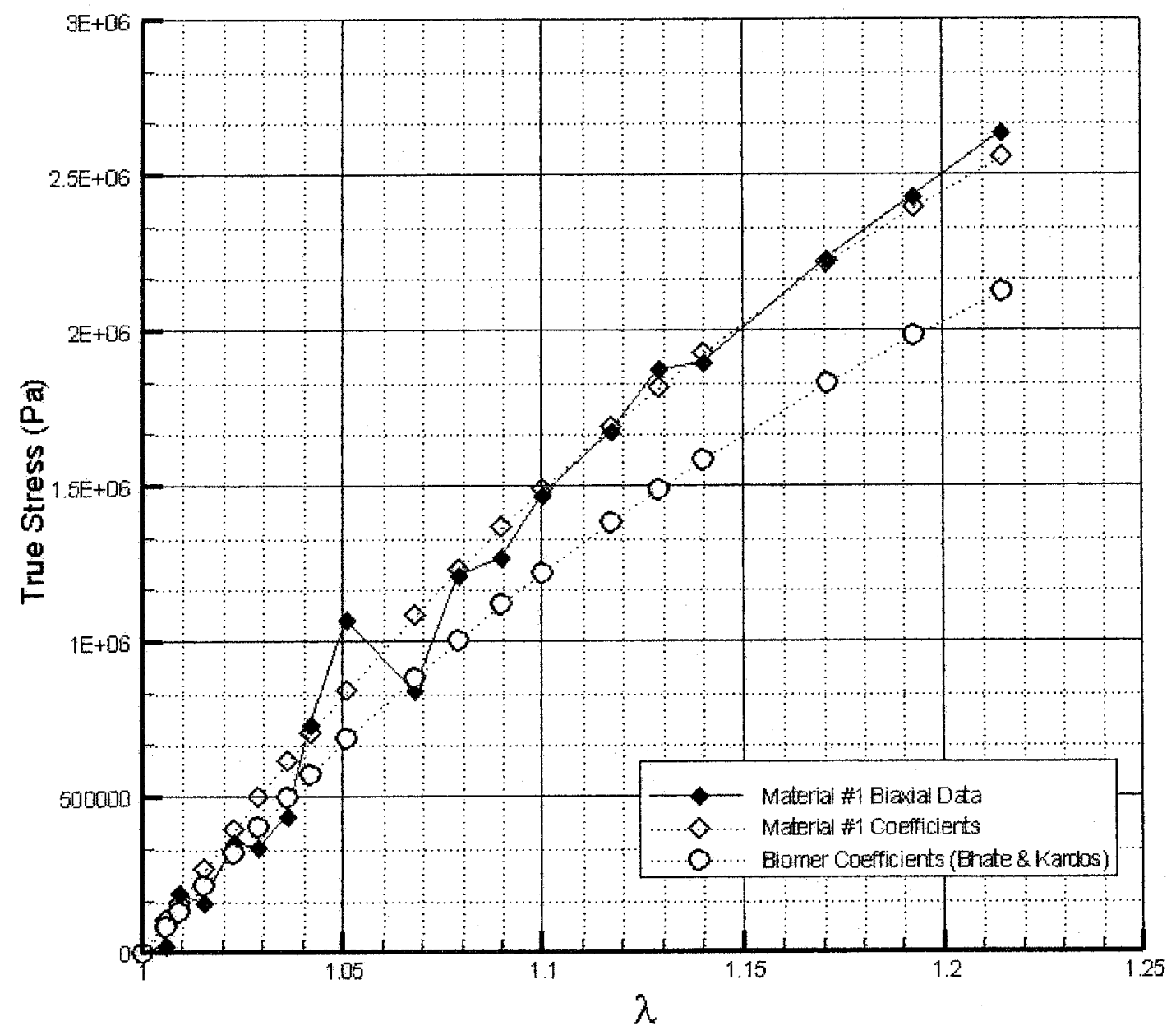

Figure 4.20: Evaluation of Mooney-Rivlin coefficients determined from least squares fit over biaxial data for Material \#1 
fluid oscillator that cycled the material prior to measurement. It is likely this softened the material. As mentioned, hysteresis in samples strained less than $50 \%$ can experience up to $20 \%$ hysteresis. Thus, it is not surprising that the Biomer stress values are $25 \%$ lower at $20 \%$ strain.

To summarize this section, Biomer data appears to be a close match to the Material \#1 and Material \#2 data for uniaxial tension up to $18 \%$ strain. For the biaxial tensile case, Biomer and Material \#1 are also in close agreement despite differences in Biomer data due to the Mullins effect.

With adequate biaxial and uniaxial hyperelastic constants available, the next step is to benchmark ABAQUS for its ability to handle hyperelastic materials under large deformations. These benchmark tests are described in section 5.3.1, however, it is first necessary to review the main obstacles to Finite Element Analysis of non-linear materials and solution procedures. 


\section{Chapter 5}

\section{Finite Element Formulation}

The preceding chapter focussed on developing a constitutive model based on the 2 parameter Mooney-Rivlin equation constrained by incompressibility. The overall variational function was developed using the principle of virtual work. The next step is to develop the finite element formulation and detail the numerical analysis. Finite element formulation is based on the Galerkin approximation. In this chapter, an undesirable peculiarity in FEA known as volumetric strain locking, which occurs due to the incompressibility constraint, is reviewed. As the diaphragm buckles, a dynamic snap-through event occurs and this is also discussed.

The finite element selected is a continuum element as it was determined that stress through the thickness was of interest. Thus, an isoparametric continuum element was selected. Both an 8 node brick and a 20 node brick element was investigated. A reduced integration scheme was incorporated to improve the speed of convergence and hybrid elements were also selected. Mixed formulation or hybrid elements, are used to resolve the problem created by the incompressibility constraint on the variational function and are explained in the next section. 


\subsection{Incompressibility constraint}

It was shown that the variational function is a relation based on strain energy that is conserved in the deforming material. If some external work is applied to the material the internal deformation energy is conserved.

Incompressible materials can have hydrostatic pressure added to them without any change in volume. No work is done by hydrostatic pressure. This can be described, alternatively, as follows: the Cauchy stress that is calculated from the variational function Equation 4.20 is based on the work done by the displacements. Despite the fact that volumetric displacements are not supposed to occur in an incompressible solid, because solution is displacement based, the volumetric stresses from the applied pressure affect the displacements and in turn the calculated Cauchy stresses become erroneous. This issue is resolved by suppressing the volumetric component of the strain field that creates the problem by independently determining the pressure stress.

For numerical analysis, the stiffness matrix maintains the Poisson ratio at $0.49999999 \ldots$ but not exactly 0.5 . In the numerical analysis, a very small change in displacement results in extremely large changes in pressure $(\operatorname{tr}(\mathbf{C})$ in the Cauchy stress). Thus, a truly displacement based analysis gives erroneous stresses ${ }^{1}$.

The constraint of incompressibility results in the elements beginning to warp as shown in Figure 5.1. Thus inaccurate stresses in the material are calculated. The essential result is too small a displacement and too large a stress. This is called volumetric strain locking.

Incompressibility is accounted for in the constitutive equation but not in the formulation of the finite element and stiffness matrix. By coupling a term that represents or is proportional to the hydrostatic pressure, a complete state of stress can be determined. ABAQUS solves this problem by determining a pressure stress, independently interpolated,

\footnotetext{
${ }^{1}$ Moreover, the ratio of shear to bulk modulus is still finite but is approaching a singularity. In the stiffness matrix, $v$ is maintained slightly compressible otherwise a singularity would exist and no convergence would occur.
} 

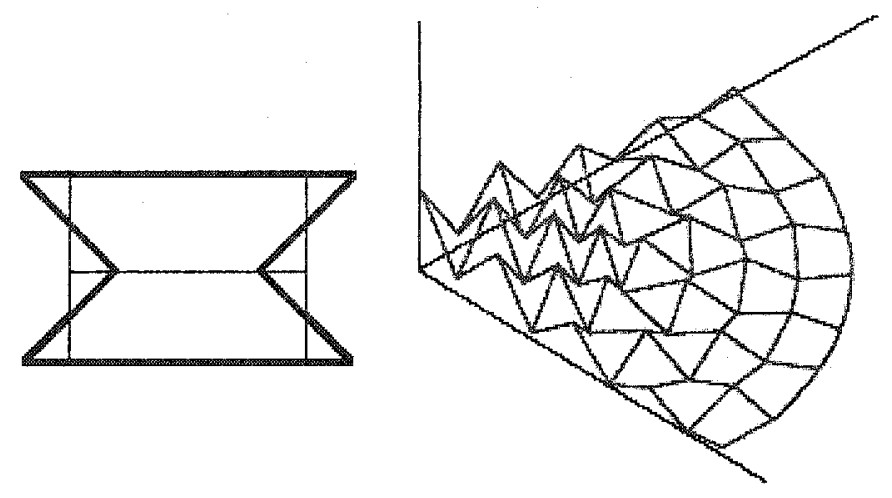

Figure 5.1: Volumetric strain locking in 2D and egg-crate warped mesh [47]

and treating it as a basic solution variable. The stress is coupled to the displacement solution through the constitutive theory and the compatibility condition by means of a Lagrange multiplier.

Proper element selection is also a concern. The elements will lock up if, due to Dirichlet boundary conditions, the element is over constrained and if there are insufficient active degrees of freedom. Lower order elements, such as 4-node isoparametric quadrilaterals for example, exhibit poor performance in non-linear analysis.

The variational relations in their simplest form following closely to the definition of Lagrange multiplier methods:

$$
\delta \bar{W}=\int_{V}\left[\bar{\sigma}: \delta \epsilon+J^{-1} \delta \lambda(\Delta p-\triangle \hat{p})\right] d V
$$

where $J$ is the volume change ratio(Jacobian) and $\delta \lambda$ is a Lagrange multiplier whose interpolation is determined [48] and $\triangle \hat{p}$ is interpolated over each element as the constraint is satisfied in an average sense [48].

The user can include the above load-stiffness correction in the analysis by selecting the hybrid elements option in ABAQUS. Hybrid elements were used in a metal plate analysis 
and were seen to improve the analysis as will be shown later.

\subsection{Numerical Procedures}

\subsubsection{Newton Approximation}

ABAQUS uses the Newton's method for solving non-linear equilibrium conditions. The basic formalism can be found in [49]. ABAQUS uses this method as convergence rates are better compared to alternate methods.

\subsubsection{Arc-Length Method}

When the diaphragm inverts it exhibits a structural instability as it snaps-through to an inverted position after approaching a limit in its deformation. This means, the loaddisplacement response shows a negative stiffness as the material buckles until it reaches a point were the structure must suddenly release strain energy to remain in equilibrium. The kinetic/thermal energy released during this snap event can be modelled as a continuous response as follows.

A typical unstable static response is shown in Figure 5.2. The load may decrease as the solution evolves as shown in load-displacement space. ABAQUS uses the modified Riks method as an effective solution of such a case. Two assumptions are made. Firstly, the loading is proportional throughout deformation: all load magnitudes vary with a scalar parameter. Secondly, the response is smooth throughout, that is sudden bifurcations do not occur. When a sudden bifurcation exists, such as in snap through behaviour, there is a discontinuous response at the point of buckling and the exact post-buckling response cannot be analyzed directly. The problem is turned into one with continuous response.

The loading during a Riks step is always proportional. 


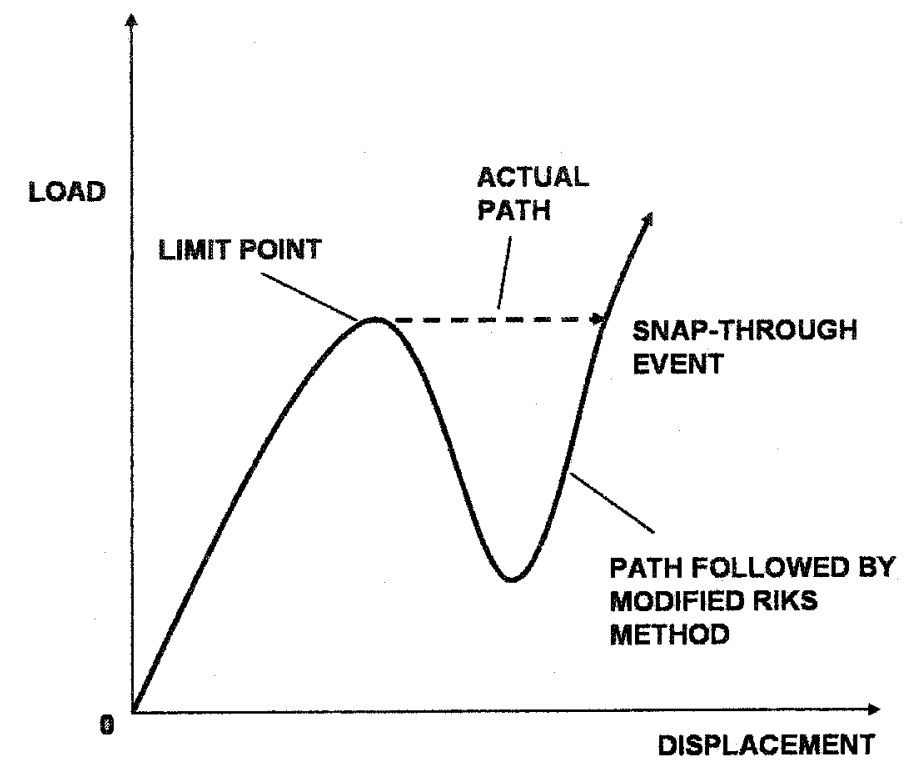

Figure 5.2: Load displacement curve for non-linear response

$$
P_{\text {total }}=P_{o}+\chi\left(P_{\text {ref }}-P_{o}\right)
$$

where $P_{o}$ is the "dead load", $P_{\text {ref }}$ is the reference load vector, and $\chi$ is the "load proportionality factor" (LPF) [50]. The LPF is found as part of the solution. In fact, the load magnitude itself is also a solution variable. Since the load and displacement are either increasing or decreasing, the LPF, which is a measure of the response, increases monotonically [50].

Given the number of issues involved in developing a model of the diaphragm deformation errors accumulating in numerical simulation, such as round off errors on each of the various approximations, Galerkins, Lagrange and Newton's methods, benchmarking the software was difficult. However with careful implementation, good results were obtained as detailed in Section 5.4 . 


\section{$5.3 \quad$ Elements}

It is appropriate at this point to discuss the type of elements available in ABAQUS and some of their characteristics.

\subsubsection{Element Shape}

It is clear to see that bending is an issue and hence the use of elements with bending capability, at low cost(time for convergence) is required. First-order triangular and tetrahedral elements are too stiff for stress analysis, and so the use of an automatic mesh generator with tetrahedral elements was avoided. Hexahedral elements in 3D analysis give the best results for minimum cost and this study looks at their performance.

\subsubsection{Element Order}

Hexahedral elements can be either 1st or 2 nd order. First order elements have 8 nodes, essentially defining the vertices or corners, of a brick element. Second order elements have 20 nodes, which include each vertex, mid-side nodes(nodes along each edge), and a single node on each face. Second-order elements provide higher accuracy for smooth problems, and capture stress concentrations more effectively. They are also better for modelling geometric features. For example, a curved surface can be modelled with few elements. Also second order elements are effective for bending dominated problems. The choice of element order would appear to lean towards using a 20 node brick for this analysis, however, the next section explains that this is not always the case.

The bending of 1 st order elements can be improved by using the incompatible modes option. The primary effect of this is to eliminate the stiffness of the element. Switching to 1 st order elements, may, however increase the computation time. 


\subsubsection{Integration Method}

Reduced integration reduces both convergence time and element assembly time, particularly in 3D problems. For example a fully integrated 20 node brick has 27 integration points, and a reduced integration 20 node element has only 8 . Full integration results in undesired volumetric locking when the material is almost incompressible, as is the case in this study. Furthermore, reduced integration has the effect of improving the accuracy of the results.

However, reduced-integration combined with second order elements also develops volumetric locking for almost incompressible materials, but only if significant straining occurs. It is not known in advance what the values of strain will be, so a 20 node element may or may not be appropriate and is investigated. This locking behaviour results in the distorted shapes as shown in Figure 5.1.

Since a more accurate approach is desired for stress analysis purposes, the switch to second order elements, greater number of nodes, is of interest and is investigated. As stated in the ABAQUS literature, second order elements are very effective in bending-dominated problems.

\subsubsection{Hybrid Elements}

It was mentioned that a load-stiffness correction is required for analysis of incompressible solids, thus, the hybrid element option was turned on in ABAQUS for all analysis, unless specified.

Reduced integration second order elements experience volumetric locking at large strains. First order elements experience poor bending behaviour unless used with the incompatible modes option. It is not known what the strains will be in the deforming diaphragm. It is beneficial to analyze the reduced integration second order elements to 
reduce cost of analysis by lowering element number as well as improving stress analysis. The next section describes efforts to assess the performance of the variety of elements.

\subsection{ABAQUS Benchmarking}

The purpose of the benchmark exercise is to assess the ability of ABAQUS to handle non-linear large deformation analysis. Axisymmetric elements, of 1st and 2nd order, were analyzed first. A 3D model with a structured hexagonal mesh was then used with 1st and 2 nd order elements. The element types were assessed on their ability to handle the large deformations. To reduce computation time, an investigation into the coarse and fine meshes were compared. A mesh quality analysis based on current hexagonal metrics was conducted. The process of benchmarking also serves to familiarize the author with ABAQUS, its analysis techniques, and its ease of use. Two types of BC's, built-in and pinned were also analyzed for their effect on the final result. The aforementioned studies were then compared with analysis and results found in the literature.

The following studies all involve inflation of a circular disk. This is the first step in benchmarking the ability of software to analyze large deflections as approached by other studies [51][52][53][17]. A measure of the solver accuracy that is often used in disk inflation analysis is the vertical deflection of the centre node of the circular plate or disk (the apex of the inflated shape). This study uses this measure of accuracy to compare the various boundary conditions, mesh densities and element types.

\subsubsection{Metal Disk Analysis}

Before attempting a rubber analysis, it is necessary to approach the problem of inflation of circular disk using a simple metal disk analysis. The purpose was to verify the boundary conditions and ensure good convergence of the solver and Newton's method. The results 
also show the effect of various boundary conditions, mesh density, and the stiffness of the elements.

The model consisted of a 1 quarter slice of a flat circular disk as shown in Figure 5.3. The diagram shows a coarse mesh $\leq 150$ elements. This study looked at a finer mesh at 620 elements and 2500 elements. This model was used for analysis of metal, rubber and Material \#1. The dimensions of 1 -quarter plate are $0.5 \mathrm{~mm}$ in thickness and $50 \mathrm{~mm}$ in radius.

The mesh was created in ABAQUS/CAE. CAE is a graphical user interface package that comes with ABAQUS that allows the user to visually observe the mesh as it is generated. The mesh is created from seeds which are essentially a rough approximation of the final nodal locations calculated by mesh generator. The seed density is entered by the user so the mesh can be concentrated or evenly spread out to allow for refinement in regions of high stress concentration. For this analysis, an evenly distributed seed density was entered to create the desired number of elements. An analysis of mesh refinement was not conducted in this study for any of the cases.

The swept mesh feature was used to generate the mesh. This requires the user to specify the direction along the peripheral edge as shown in Figure 5.3 for the swept mesh to occur. The swept mesh feature requires the use of wedge elements at the apex of or centre of the dome or circular shape. With this option, it was possible to generate a structured mesh of hexagonal and wedge elements both of 1 st or 2 nd order.

The metal of high elastic modulus and poisson ratio was entered into the ABAQUS solver, $2 \mathrm{e} 11 \mathrm{~Pa}$ and $\mathrm{v}=0.29$. On one side of the disk, an arbitrary pressure of $30000 \mathrm{~Pa}$ $(100 \mathrm{mHg})$ was applied and the resulting finite element deflection was observed. A course mesh of 150 elements was applied. The boundary conditions at the centre and the edges of the quarter plate 3D model are shown in Figure 5.4. As can be seen in Figure 5.4, the nodes at the edge of the plate were built-in, in other words, displacement in 3 principal 


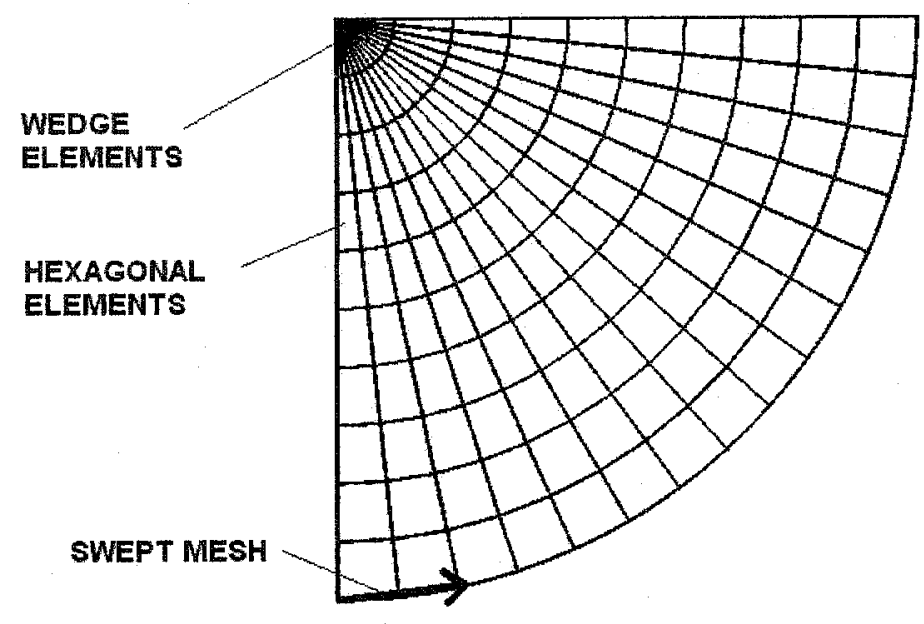

Figure 5.3: Thindisk model used in study with coarse mesh $\leq 150$ elements

directions, $\mathrm{x}, \mathrm{y}$ and $\mathrm{z}$ were set to 0 .

A calculation of defiection in a metal plate under the same loading conditions and with the same dimensions as mentioned earlier, was conducted in MathCAD. The calculation is based on thin plate theory. The theory assumes a clamped edge at the periphery. The details of the calculation are located outlined in the Appendix A. The boundary conditions in ABAQUS for the $3 \mathrm{D}$ model involved constraining all nodes on the peripheral edge of the model as shown in Figure 5.5. The condition is built-in where the displacements in the 3 principal directions are zero. As shown in Figure 5.5 both bottom, top and mid side nodes in contact with the grey region shown in the Figure are constrained. The Figure shows one condition where the nodes on the bottom row are constrained, and, in this study, one simulation was conducted as such. This produces erroneous results. In all other simulations, all nodes along the edge are built-in unless stated otherwise.

An analysis of a metal plate conducted for different 1st and 2nd order hexagonal elements is given in Table 5.1. The first column shows results for clamped or built-in conditions for all nodes along the periphery. The last simulation shows results for constraining only the bottom row of nodes. 

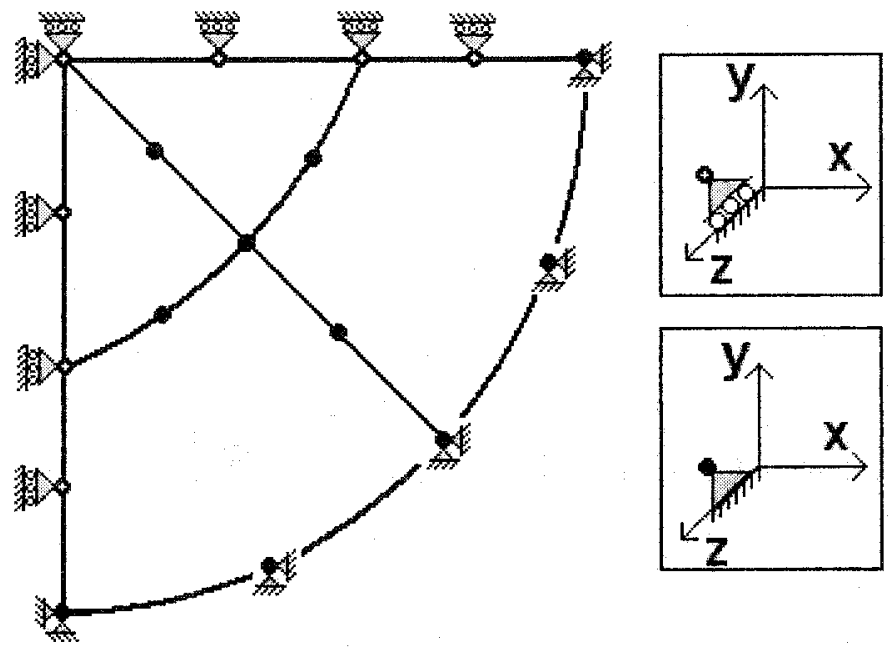

Figure 5.4: Boundary conditions for thin disk model. Filled circles indicate no motion in Z-direction

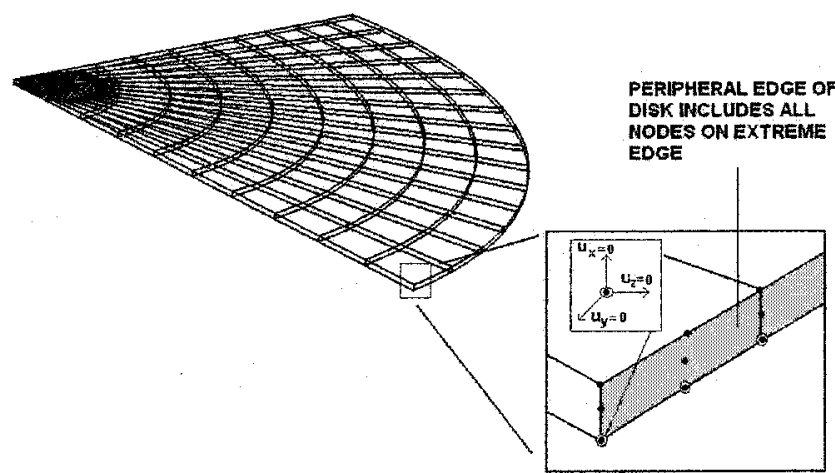

Figure 5.5: Meshed disk with inset showing periphery of disk with built-in, boundary condition along bottom row of nodes 


\begin{tabular}{|l|l|c|c|}
\hline Built-In Boundary Condition & Element Type & Number of Elements & Deflection at Centre \\
\hline THEORY (BUILT IN) & - & - & $1.288 \mathrm{E}-3$ \\
\hline ALL NODES & C3D8R & 150 & $0.703 \mathrm{E}-3$ \\
\hline ALL NODES & C3D8R HYBRID & 150 & $0.762 \mathrm{E}-3$ \\
\hline ALL NODES & C3D20R & 160 & $1.262 \mathrm{E}-3$ \\
\hline ALL NODES & C3D20R HYBRID & 150 & $1.290 \mathrm{E}-3$ \\
\hline ALL NODES & C3D20R HYBRID & 620 & $1.286 \mathrm{E}-3$ \\
\hline ALL NODES & C3D20R HYBRID & 2500 & $1.2861 \mathrm{E}-3$ \\
\hline BOTTOM EDGE ONLY & C3D20R HYBRID & 150 & $0.802 \mathrm{E}-3$ \\
\hline
\end{tabular}

Table 5.1: Deflection at centre for metal disk analysis using various element types and density

1st order, 8-node brick elements were analyzed first. The result from these elements underestimates the hand calculation of the deflection height of the center of the circular disk. This is the result of the 8-node brick being too stiff as the adjustment to a 20 node brick shows.

A second order element, C3D20RH, was used for the same coarse mesh and an acceptable result was obtained. Further refinement of the mesh produced little effect on the accuracy of the result as can be seen in the table. The last row of Table 5.1 shows the results for a different boundary condition as shown in Figure 5.5.

The stress distribution in the metal plate was also analyzed using the MathCAD simulation. The results from the ABAQUS simulation for the 150 element, 20 node brick are superimposed on the theoretical calculation and are shown in Figure 5.6. The results indicate that the radial stresses are lower near the boundary compared to the MathCAD analysis. The constraint in the boundary shows a $50 \%$ lower stress in the ABAQUS simulation.

As mentioned earlier, 1st order elements with incompatible modes can improve the bending stiffness of the element. This may improve the results for 1st order element 


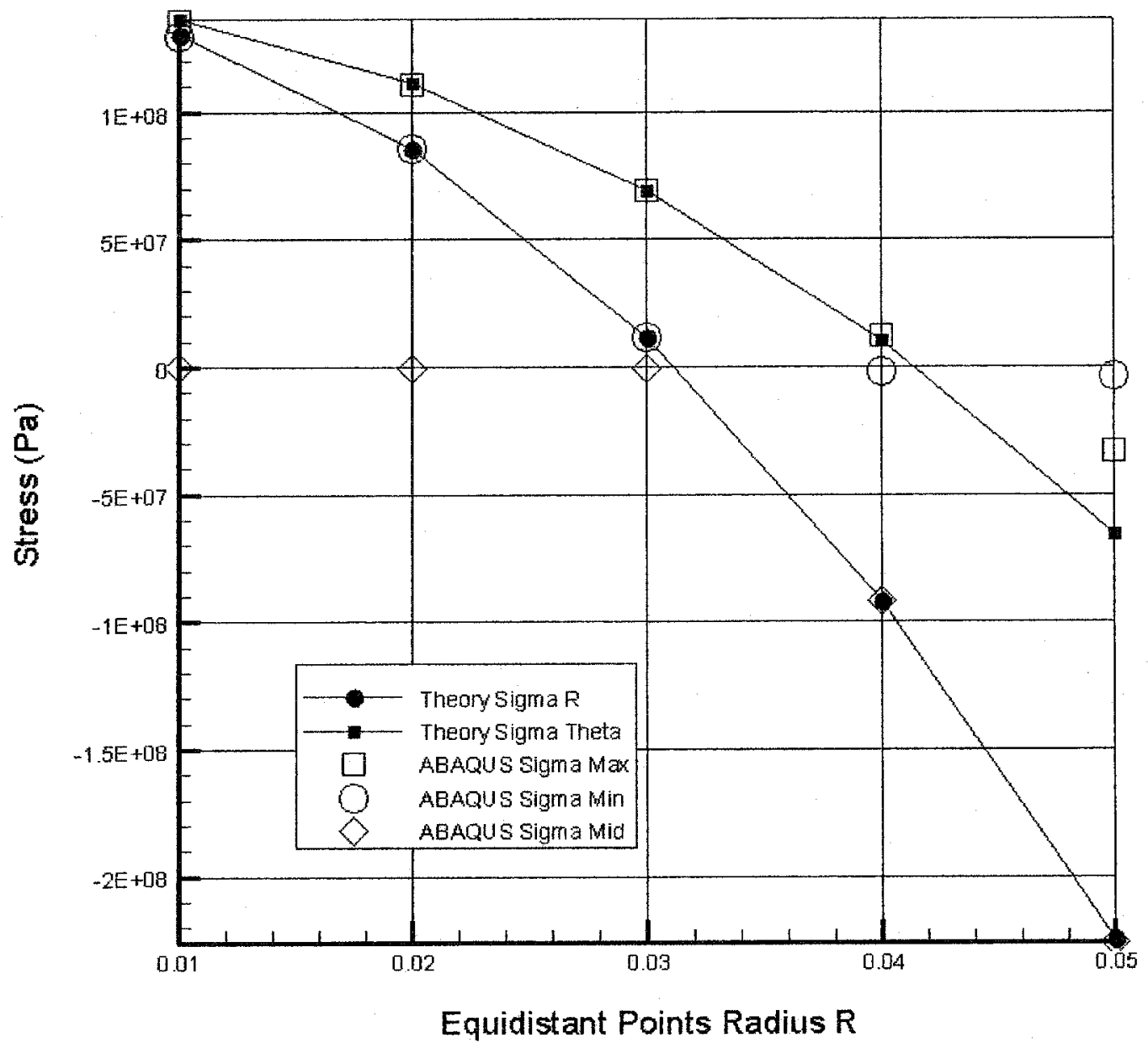

Figure 5.6: Stress distribution at equally spaced nodes along the pressurized metal disk at $30 \mathrm{KPa}$ 
analysis but was not investigated in this study. In summary, the 20-node reduced integration element (C3D20RH) shows excellent correlation with the results from the MathCAD simulation as well as the deflection.

This completes the small-deflection analysis. The next stage is to compare simulation results from ABAQUS with a rubber-like hyperelastic material.

\subsubsection{Rubber Disk Analysis}

The previous analysis showed the response of high elastic modulus steel plate to pressurization. The next 2 models analyzed are also circular disks but use published 2-term Mooney-Rivlin coefficients from 2 previous rubber inflation studies.

The first study involves a thin disk of similar dimensions to the metal disk defined in the last section. It was decided, as a first approach to model this thin disk with second order axisymmetric elements. Two boundary condition types are analyzed.

A second study models a thick rubber disk that is also available in the ABAQUS benchmarks manual. This manual give results for simulations in ABAQUS/Standard for a variety of 1 st order element types for this thick disk model. It was decided to model this disk with 3D continuum elements using the swept mesh technique and evenly distributed elements, ie. no mesh refinement in potential regions of stress concentration.

\section{Axisymmetric Analysis}

The thin disk model was obtained from Oden's text, Finite Element of Non-Linear Continua [16]. The model compares results from the Hart-Smith and Crisp model [17]. In this analysis, a circular disk of radius $2.54 \mathrm{~cm}$ and $0.2 \mathrm{~mm}$ thickness was analysed. The Mooney-Rivlin coefficients used in their analysis were $C_{01}$ and $C_{01}$ values, given by Oden for this analysis, as $931631 \mathrm{~Pa}$ and $171616 \mathrm{~Pa}$, respectively. Results were analyzed by Oden and they show that if a distributed pressure load of $951245 \mathrm{~Pa}$ is applied to one side of the 

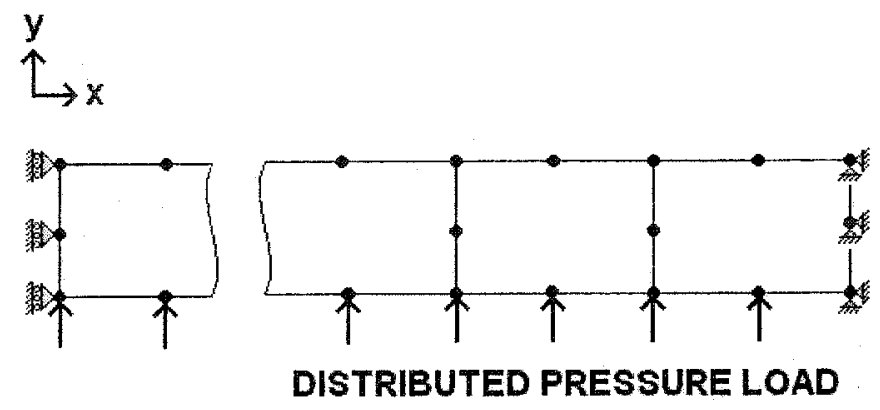

\section{BUILT IN}

Figure 5.7: Built-in boundary conditions for axisymmetric model

disk, the observed deflection would be $15 \mathrm{~cm}$ at the apex of the inflated shape and the finite element error relative to experimental results would be $\pm 6 \%$.

The diaphragm will not see the type of loading, as seen in this model ( $\sim 7000 \mathrm{mmHg})$. Furthermore, given the much lower Mooney-Rivlin coefficients stated, the amount of strain in the material at this deflection is high. Nevertheless, despite the high loads and the large strains associated with such a model $>300 \%$, an attempt was made to use ABAQUS to recreate this situation. A total of 51 elements were distributed evenly at distances across the axisymmetric segment of the model. This resulted in elements $0.468 \mathrm{~mm}$ in width and $0.2 \mathrm{~mm}$ thick. Eight node (second order) axisymmetric elements with reduced integration, were used. The $C_{01}$ and $C_{01}$ values were $931631 \mathrm{~Pa}$ and $171616 \mathrm{~Pa}$ respectively. A distributed pressure load was applied to the model at $951245 \mathrm{~Pa}$.

The analysis was performed with two types of boundary conditions as shown in Figures 5.7 and Figure 5.8. The built-in constraint is similar to that of the metal disk model. The pinned constraint consists of pinning the mid-side node of the last quadratic elements and constraining the top and bottom nodes, shown in the Figure 5.8 as 1 and 2. By applying the equation constraint, the edge is allowed to rotate while still allowing edge to become thinner, ie. the thickness deformation of the diaphragm. 


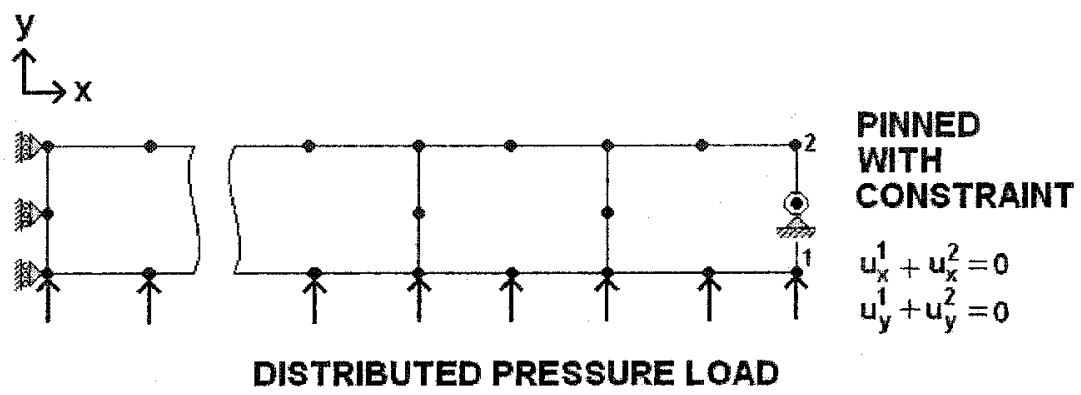

Figure 5.8: Pinned boundary conditions for axisymmetric model

The results show that the relaxed boundary condition of the pinned constraint produces a higher deflection versus, the built-in constraint. The built-in constraint results in a distortion of the elements near the edge as the elements only bend so far before turning in on themselves. In the pinned condition and with the constraint equation, the result is a much higher deflection as expected. Although these were second order elements with reduced integration, they appear to deform well, with no apparent volumetric locking. It is possible to observe this as the locking will show a checker pattern effect where the pressure stresses and strain values will alternate from large to small to large again instead of a smooth monotonic transition in field values from one element to the next. Furthermore, it was observed in the pinned condition simulation that the element with the edge constraint, distorts up to $300 \%$ and there was no apparent volumetric locking in that element. Note that the 1st order incompatible modes produce the same result for the large deflection analysis with a pinned boundary condition. This indicates that the 1st order elements can handle the same deformation, for the same mesh, if used with incompatible modes.

In summary, this simulation provides a quick estimate of the ability of ABAQUS to handle large deflection analysis and also shows the significant impact of the differences in boundary conditions. The built-in boundary conditions can lead to more errors if used with finite elements. 


\begin{tabular}{|c|c|c|c|}
\hline Modelling Method & Element Type & Boundary Condition & Deflection at Centre \\
\hline Oden Theory & - & - & $15 \mathrm{~cm}$ \\
\hline ABAQUS & CAX8RH & Built-In & $13.3 \mathrm{~cm}$ \\
\hline ABAQUS & CAX8RH & Pinned & $17.7 \mathrm{~cm}$ \\
\hline ABAQUS & CAX4RI & Pinned & $17.8 \mathrm{~cm}$ \\
\hline
\end{tabular}

Table 5.2: Deflection at centre for Hart-Smith and Crisp axisymmetric model

\section{D Modelling}

A thick disk analysis with experimental results is available from Oden [16]. This rubber inflation test is a much larger and thicker rubber disk than the previous model, however, with this model, data is available at varying increments of applied pressure up to the maximum pressure. Thus, this is a more suitable model for comparison as ABAQUS convergence can be assessed at much lower strains versus the high strain analysis in the previous model. In this analysis it was decided to provide an assessment of the 1st order elements and, particularly, the incompatible modes option.

The model consists of a circular disk of radius $19.05 \mathrm{~cm}(7.5 \mathrm{in})$ and $1.27 \mathrm{~cm}(0.5 \mathrm{in})$ thickness. For the 3D analysis, Oden gives the two Mooney-Rivlin coefficients, $C_{10}$ and $C_{01}$, with values of $551581 \mathrm{~Pa}(80 \mathrm{PSI})$ and $137895 \mathrm{~Pa}$ (20PSI), respectively.

As in the metal disk analysis, the swept mesh feature was used to generate the mesh. The swept mesh feature requires the use of wedge elements at the centre of the disk. A structured mesh of hexagonal and wedge elements, both of 1st order (8-node) elements, C3D8IH, C3D8RH, were analyzed. Two types of first order elements were analyzed for their response: reduced integration and incompatible modes. As mentioned earlier, incompatible modes was a "fix" for 1st order elements to improve their bending behaviour.

The results are given in Figure 5.9.

The incompatible modes graph and the reduced integration both show excellent cor- 


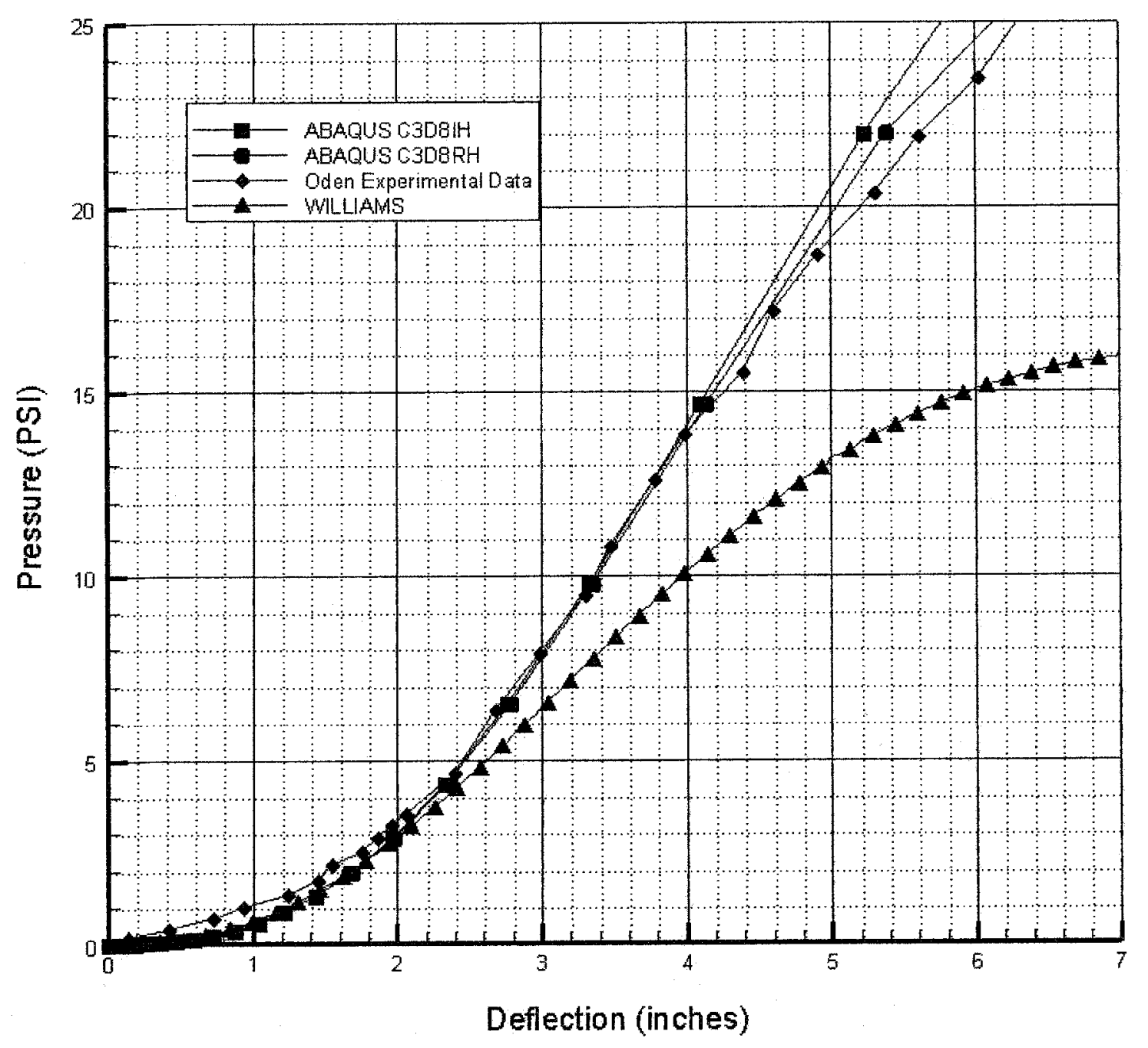

Figure 5.9: Thick rubber disk analysis 
relation up to 5 inches of deflection, after which the C3D8IH elements show a deviation from the observed data provided by Oden. It is important to note that at low deflection the both element types underestimate the Oden data. In summary the incompatible modes appears to have good correlation until the curve is taken into higher strains and the non-linear regions at which point the curve shifts upwards. The C3D8RH curve show's a better correlation with the experimental data. It is also possible that leakage or permanent plastic deformation, or viscoelastic effects in the experimentation are not accounted for in the model and is the cause of the discrepancy. If 1st order elements are to be used they must still be assessed under the different modes of deformation.

Also shown in this figure is the Williams [54] calculation which is described in detail in the following sections. It provides a hand calculation of the inflation of a rubber disk, but is only suitable up until small deflections as is described in the following section.

Hand Calculation: William Model A pseudo-hand calculation was conducted using power law relation over the stress-strain curve as presented by Williams [54]. With this model, deflection height of an inflating disk can be estimated as well as the thickness reduction in the material. A complete derivation can be found in Williams's text [54]. The model uses a power-curve fit. The results appear to be valid over a low range of deflections as the power curve fit is similar to that of the Mooney-Rivlin model in that it follows the curve up to the more linear region of the full elastomeric curve, as shown in Figure 4.7. To generate a stress-strain curve that the Williams model can use, a simple uniaxial case is evaluated in ABAQUS and the stress-strain response is output to a file. The stress-strain data is plotted and a power curve is fit to the data using Tecplot and the power curve coefficients can be determined. The deflection is solved from the equation iteratively using an Excel spreadsheet solver function. An example of the spread sheet is shown in the Appendix B. 


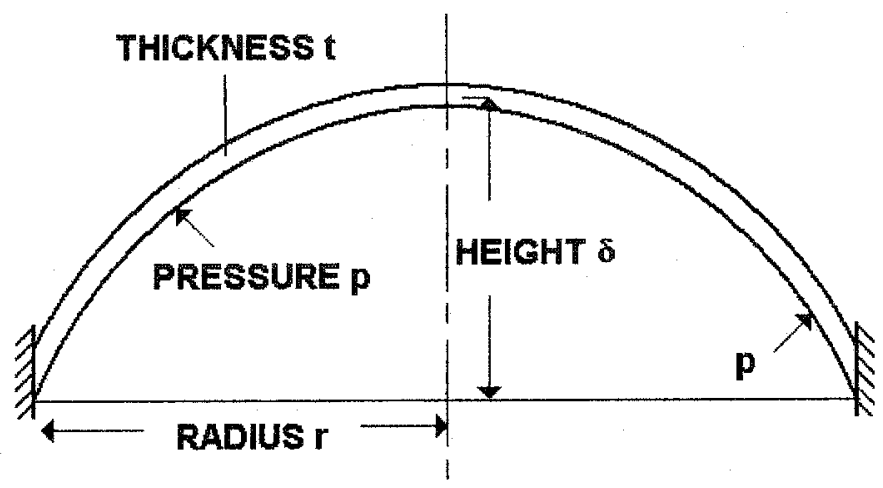

Figure 5.10: Assumed shape of cap of sphere used in Williams model.

The pressure-deflection curve can be determined from the following equation:

$$
\frac{\operatorname{Pr}}{2 t a}=\frac{\left(\ln \left(1+\frac{\delta^{2}}{a^{2}}\right)\right)^{b}\left(2 \frac{\delta}{a}\right)}{\left(1+\frac{\delta^{2}}{a^{2}}\right)^{2}}
$$

where the power curve coefficients, $a$ and $b$ in Equation 5.3 are defined by:

$$
\sigma=a \varepsilon^{b}
$$

and the other variables in Equation 5.3 are defined in Figure 5.10.

Thus, with 2-term Mooney-Rivlin coefficients and dimensions provided for the thick disk model above, pressure versus deflection data can be created. The results of the deflection at various pressures is also shown in Figure 5.9. There appears to be a good match against results for a deflection height up to 2.5 inches.

The Williams model works well with thick disks, however, the values generated for models with low thickness, $0.5 \mathrm{~mm}$ or less, were significantly lower than the finite element analysis as will be shown in the next section.

In summary, the rubber analysis shows that both types of 1st order elements, C3D8RH and C3D8IH, are capable of representing experimental data using 2-term Mooney-Rivlin coefficients provided in the literature. It also stands to reason that second order elements 
are also capable of representing the same behaviour, at reduced cost, however, checking for volumetric locking in the final model in highly constrained regions is necessary.

\subsubsection{Polyurethane Disk Analysis}

\section{D Modelling}

Before proceeding with the actual model, an assessment of the behaviour of the elements with an even stiffer material, polyurethane was tested. To do this, the original metal disk model was used again with the same mesh density and boundary conditions. The entire data range available for Material \#1 was entered into ABAQUS for the uniaxial tensile case and the coefficients produced by the least squares method in ABAQUS are $C_{10}$ and $C_{01}$ values, -920340 and 2962931 respectively, as was presented in Section 4. The boundary conditions are the same as the metal disk analysis shown in Figure 5.4. The purpose was to compare the response of both the 1st and 2 nd order elements to pressurization of a thin plate. Figure 5.11, shows the response of both rubber and Material \#1 in the same model and element type. There was no significant difference between the 1st and 2 nd order elements to the pressurization. The analysis of a thin rubber disk model was also conducted with Mooney-Rivlin coefficients of $C_{10}$ and $C_{01}, 551581 \mathrm{~Pa}$ (80PSI) and $137895 \mathrm{~Pa}$ (20PSI) respectively.

Also shown is the Williams hand calculation. It is noted that the Williams model is sufficient for the rubber and the values were even lower with the more stiffer Material \#1 coefficients. For example, when compared at an arbitrarily low pressure of $100 \mathrm{mmHg}$ $(13,000 \mathrm{~Pa})$, as much as $30 \%$ difference was observed between the hand calculation and the FEA results for thin rubber models. The results of the Williams simulation are also shown in Figure 5.11.

The stress distribution in both the rubber and Material \#1 plate was also performed in 


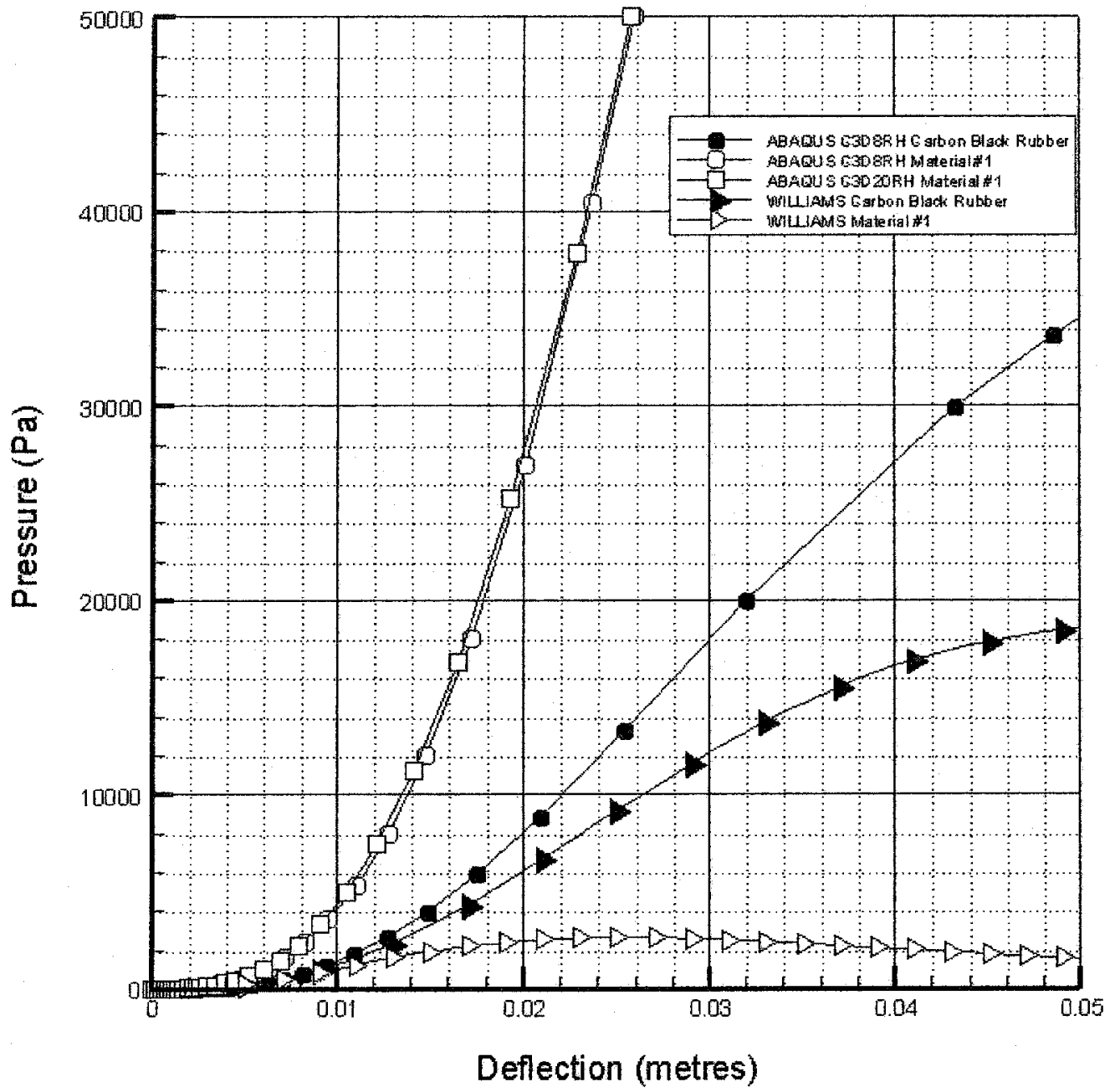

Figure 5.11: Comparison of response of 1st and 2nd order elements. Rubber is superimposed for comparison 


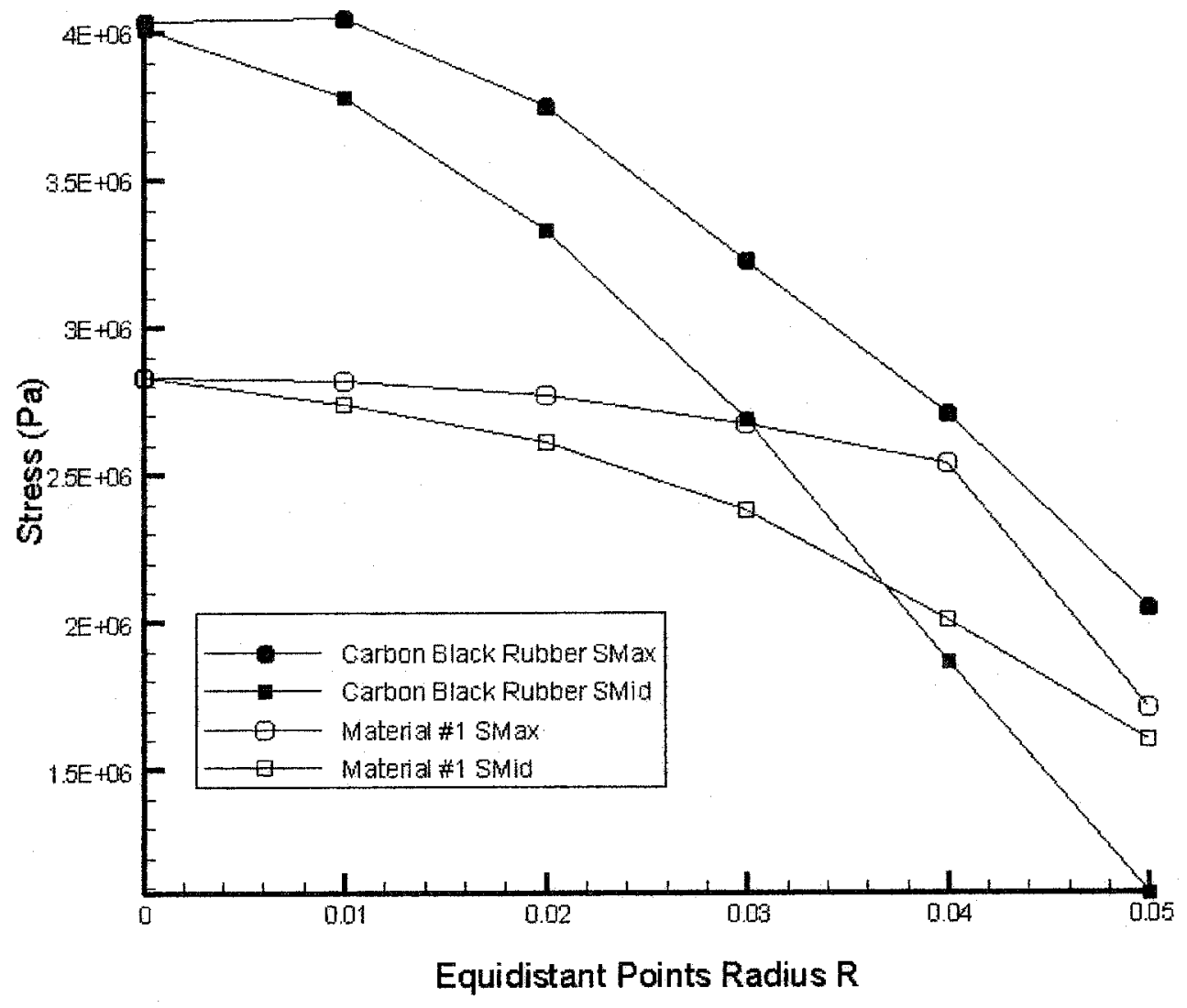

Figure 5.12: Material \#1 disk stress analysis for second order, C3D20RH, elements at $30 \mathrm{KPa}$. Rubber analysis is superimposed. 
a post-processing analysis. The results are from the 150 element, 20 node brick elements subjected to $30 \mathrm{KPa}$ of distributed pressure. The results are shown in Figure 5.12. Plotted on this graph are the maximum principal stresses and the mid-stresses. The maximum principal stress is essentially the hoop-stress in rectangular co-ordinates. Clearly, for the same applied pressure, the rubber experiences higher stress distribution than Material \#1. Also a stress distribution pattern is observed where, similar to the metal disk, the greatest stresses are at the apex of the diaphragm and become less and less near the extreme periphery.

In summary, the boundary condition of built-in is sufficient for the small strain analysis in our model. This is shown by the analysis of the thick disk model where the bending of the elements, near the periphery, gives a smooth response at low strains and pressures and are not observed to distort. Furthermore, the simulation appears to match closely with the Oden's experimental results as shown in Figure 5.9 which has the disk built-in at the periphery. Volumetric locking did occur in the axisymmetric, Hart-Smith and Crisp model which was subjected to high pressures. However, it is important to note as this was an investigation conducted earlier in the development of this project was not the most appropriate case as it involved excessive deformation, $>300 \%$. Also, the case looks at a centre of deflection height at one maximum pressure, whereas the thick 3D model looks at various pressures. With the excellent correlation in the thick model, built-in boundary condition is sufficient for the strain range, $<50 \%$, expected in the diaphragm model.

Overall, for the thick 3D model, 1st order elements follow the centre of deflection of Oden's experimental results with the thick disk very well. This result indicates that the hybrid, brick element with reduced integration can represent deflections at various pressures very well.

The next step is to analyze a thinner model and to verify whether higher order elements can model the pressures and distortions in the diaphragm. Proceeding with the inflation 
of a flat thin disk model for rubber and Material \#1, the 1st and second order elements are compared. The results indicate that second order elements with Material \#1 coefficients, do not experience apparent volumetric locking distortion within the range of pressures applied, $<30 \mathrm{KPa}$ for the thin model. As mentioned earlier, volumetric locking can be observed with the quilt pattern display option in the contour plot of the pressure field value.

Thus, in the efforts to save computational cost, by reducing element number, it is recommended that the reduced integration, second order element, C3D20RH is chosen for the diaphragm model used in this study. Furthermore, for the relatively low strains expected in this study, the built-in boundary condition is sufficient rather than the proposed pinned boundary condition given by the ABAQUS benchmark manual.

The built-in boundary condition and the second order element C3D20RH, is applied to the model of a cast diaphragm, and is detailed in Chapter 6 . 


\section{Chapter 6}

\section{Diaphragm Model}

A typical diaphragm shape is analyzed as a first approach. The proposed shape is based upon measurements from previous HSVAD diaphragms, although, the shape of the diaphragm is simplified to be circular and geometrically axisymmetric. An actual CAD drawing of a potential candidate diaphragm shape was unavailable at the time of this study. However, it is possible to import a final part into ABAQUS/CAE and create the desired mesh. The diaphragm model in the Jin and Clark study [55], for example, is similar to this design.

Geometry The overall shape of the diaphragm appears like the deformed shape of the thin inflated disks. It is a dome shape with a centre height of $20 \mathrm{~mm}$ and a radius of $50 \mathrm{~mm}$. Once again, a 1 quarter piece of the diaphragm is modelled as it is axisymmetric. An isometric view of the diaphragm is shown in Figure 6.1.

The model used to represent the diaphragm in its final shape is slightly thicker at $0.83 \mathrm{~mm}$ than the flat disk model at $0.5 \mathrm{~mm}$. This was the thickness observed in the diaphragms used in the inflation test.

The diaphragm is modelled with a lip at the extreme periphery. The lip represents the diaphragm-housing junction where only the nodes on the edge face are subjected to the 


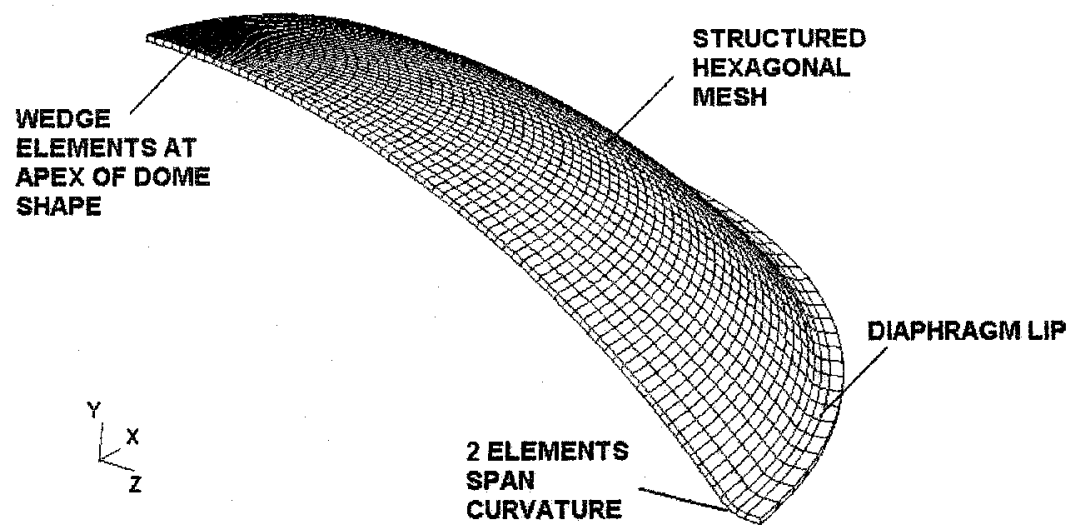

Figure 6.1: Isometric view of diaphragm model

constraint as shown also in thin disk model in Figure 5.5. The edge turn has a radius of curvature of $2.56 \mathrm{~mm}$. It is expected that this radius of curvature will create high strains in the final deformed shape and a potential source for volumetric locking in the analysis. The nodes in the region are refined such that two elements span the curvature of the diaphragm lip.

Boundary Conditions The results from the previous section indicate that a built-in boundary condition is sufficient for this analysis. The boundary conditions are as shown in Figure 5.4 .

Material Properties Raw data from the uniaxial and biaxial material tests of Material \#1 were entered using the ABAQUS data entry option. Uniaxial stress-strain data from $15 \%-40 \%$ and biaxial stress-strain data from $5 \%-22 \%$ was entered into the ABAQUS simulation. The data entered must be nominal stress-strain. As the biaxial data is true stress, this must be divided by $\lambda$ to get the nominal stress values. The uniaxial data is already in terms of nominal stress values. The coefficients can be evaluated in ABAQUS using the evaluation option in the materials window. The resulting coefficients from this 


\begin{tabular}{|c|c|}
\hline Stress State & Stability Limit \\
\hline Uniaxial Tension & 6.01 \\
\hline Uniaxial Compression & -0.95 \\
\hline Biaxial Tension & 3.96 \\
\hline Biaxial Compression & -0.62 \\
\hline Planar Tension & 5.94 \\
\hline Planar Compression & -0.85 \\
\hline
\end{tabular}

Table 6.1: Strain ranges within which stable response is achieved in ABAQUS for entered raw data

evaluation are $C_{10}=1392246$ and $C_{01}=-28395$. It is uncertain what mathematical approximation method ABAQUS uses to converge upon these coefficients from the raw data. ABAQUS also performs an evaluation of the data and produces strain values within which a stable response is achieved. With the data ranges entered as in this study, the strain limits are given in Table 6.1.

The coefficients generated by ABAQUS were evaluated using the stress-strain Equations 4.32 and 4.33 as before. The responses are shown in Figures 6.2 and 6.3 .

Mesh and Elements The elements used in this model are 2nd order, 20-node bricks with reduced integration, C3D20RH. At the apex of the dome shape, the swept mesh automatically generates 15 -node wedge elements. The mesh was generated using the swept mesh option in ABAQUS/CAE. The final mesh consisted of 2304 elements and 21099 nodes. Such aa dense mesh was necessary in order to create a mesh of adequate aspect ratio along the curved geometry, as well as generate a refined mesh in the region of the curvature of the diaphragm lip. Overall, the computation time takes from 5-9 hours.

\subsection{Results}

A negative pressure was applied to the surface of the model pushing against the diaphragm and causing inversion. The magnitude of pressure applied was $30 \mathrm{KPa}(225 \mathrm{mmHg})$. 


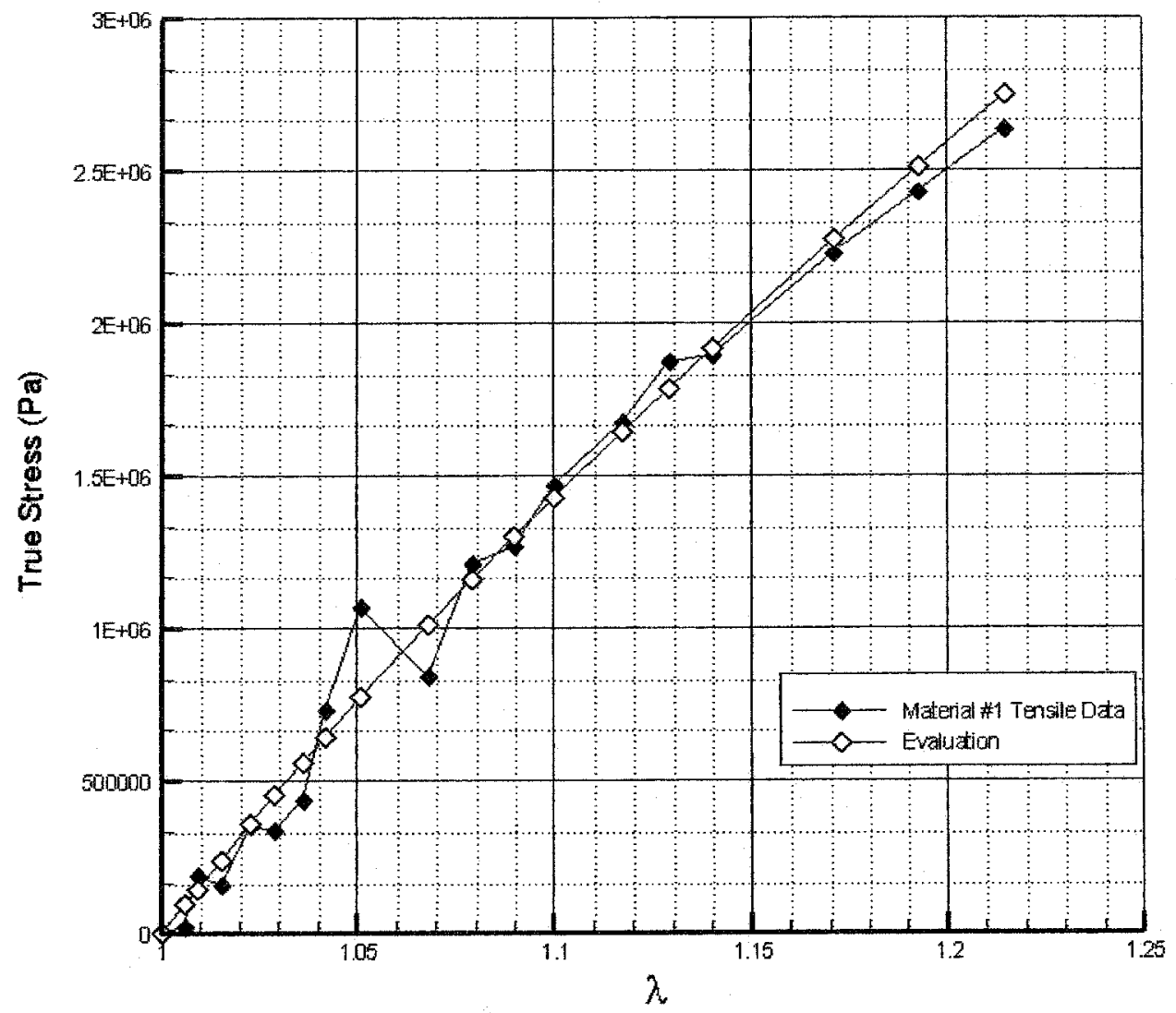

Figure 6.2: Evaluation of ABAQUS generated coefficients in biaxial tension 


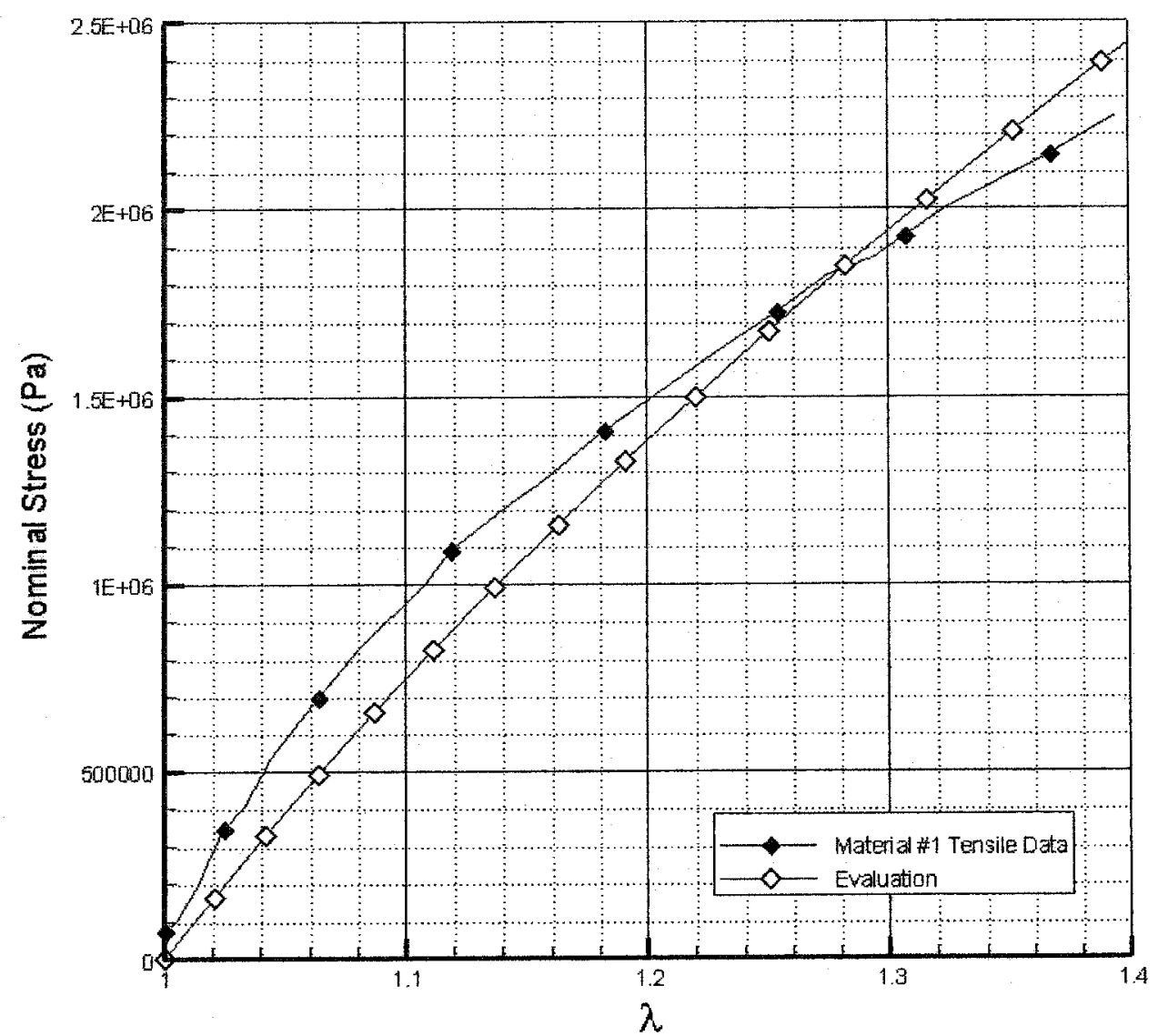

Figure 6.3: Evaluation of ABAQUS generated coefficients in uniaxial tension 

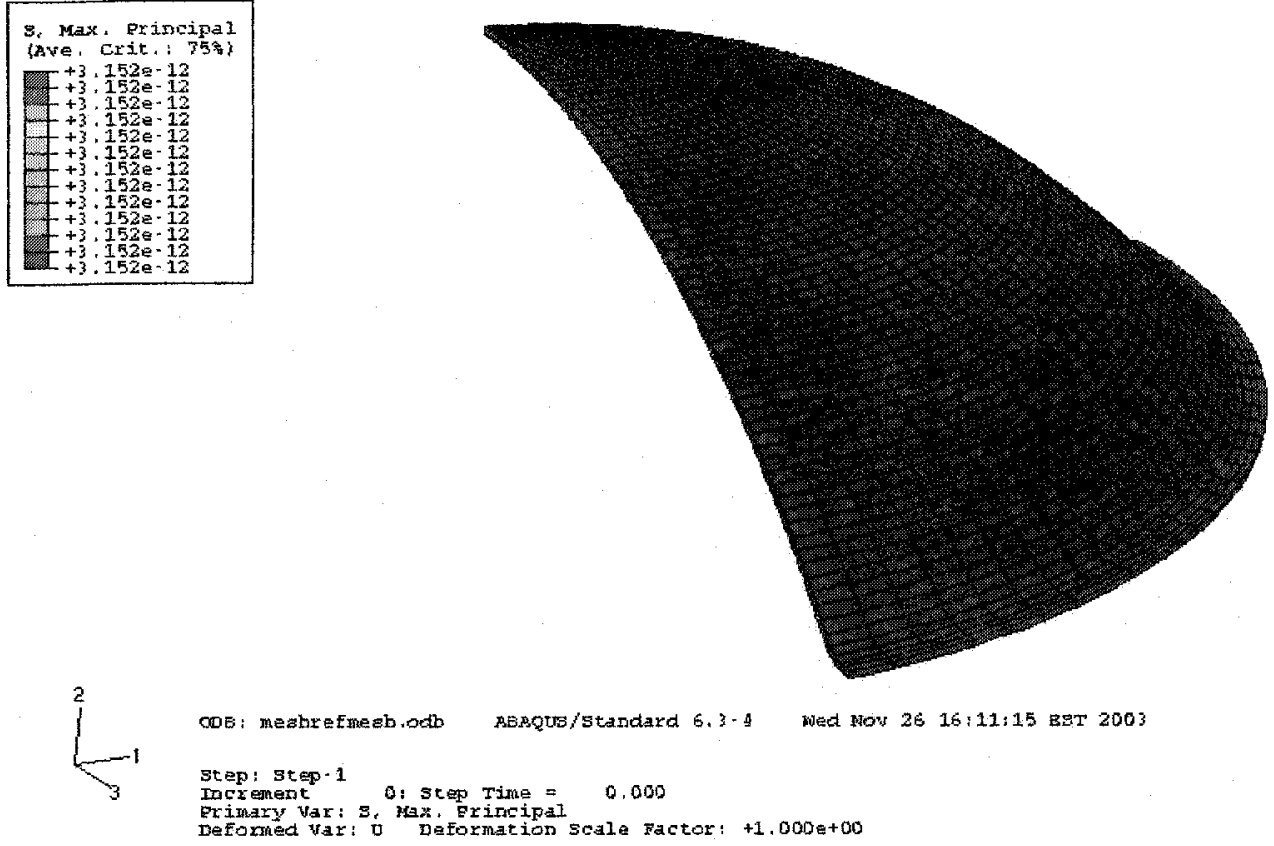

Figure 6.4: Deformation at $0 \mathrm{KPa}$

The resulting centre of deflection from zero or from the $\mathrm{X}-\mathrm{Z}$ plane as shown in Figure 6.1, is $-6.88 \mathrm{~cm}$ for the inflation after inversion. This is a reasonable value given the size of the diaphragm $($ diameter $=10 \mathrm{~cm}$ ) and the original height of the undeformed diaphragm model which is $2 \mathrm{~cm}$.

For the negative pressure model, the simulation shows that the center is depressed first and the depression grows until the diaphragm reaches a limit point and flips over, as expected. The deformed shape at intermediate pressures are shown in Figures 6.4-6.9.

The stresses as the depression propagates along the diaphragm are low. At an arbitrary pressurization of $21 \mathrm{KPa}$, Figure 6.7 , the maximum principal stress is at a maximum of $700 \mathrm{KPa}$. This changes dramatically when the diaphragm is flipped over and the stress values increase by a magnitude of 10 when the diaphragm is allowed to stretch. After inversion, at a pressurization of $27 \mathrm{KPa}$, the maximum stress in the material, if the material is allowed to stretch, is greater than $5 \mathrm{MPa}$ and is concentrated at the periphery of the 

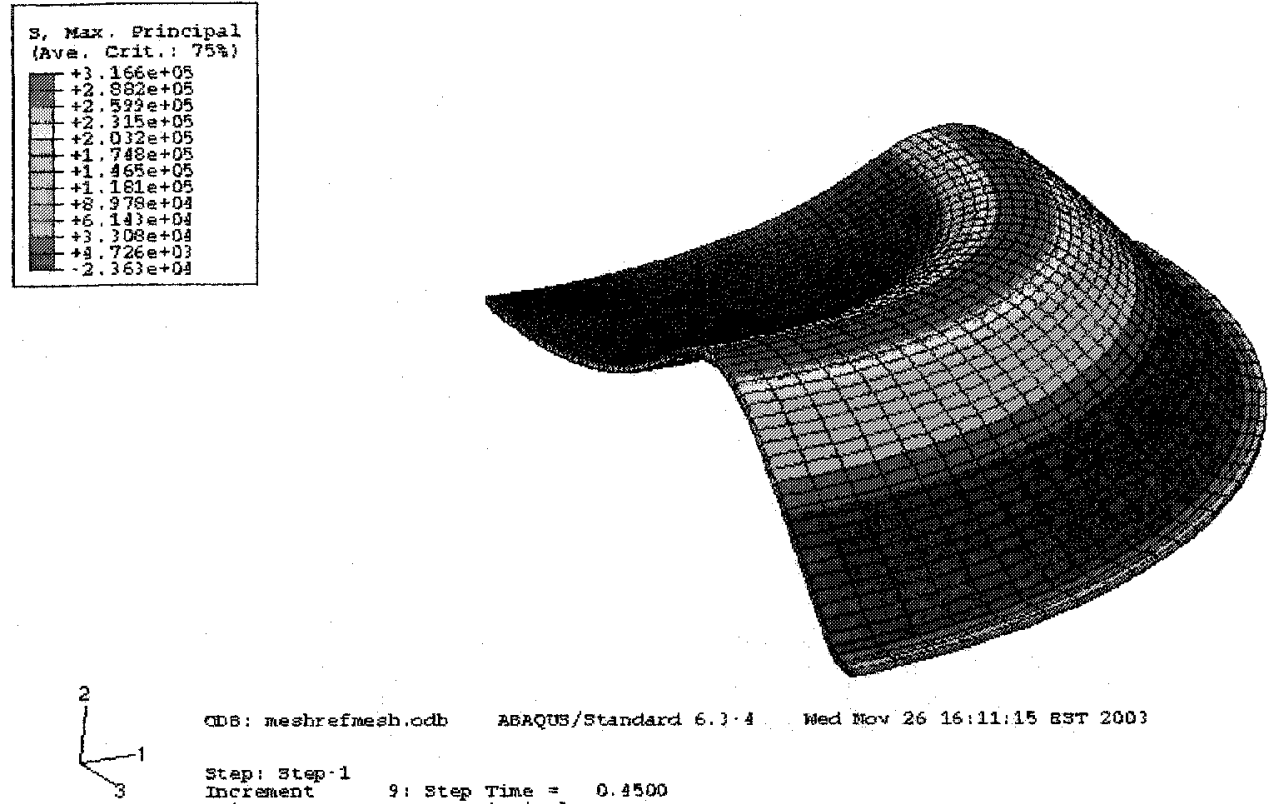

Wo: meshr efjuesh.od

MERQQUS/Standard $6.3 \cdot 4$

Hed noy 26 15:11:15 $\mathrm{BST} 2003$

Step: 3 teg-1

9: step Time $=0.4500$

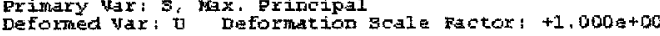

Figure 6.5: Deformation at $7 \mathrm{KPa}$
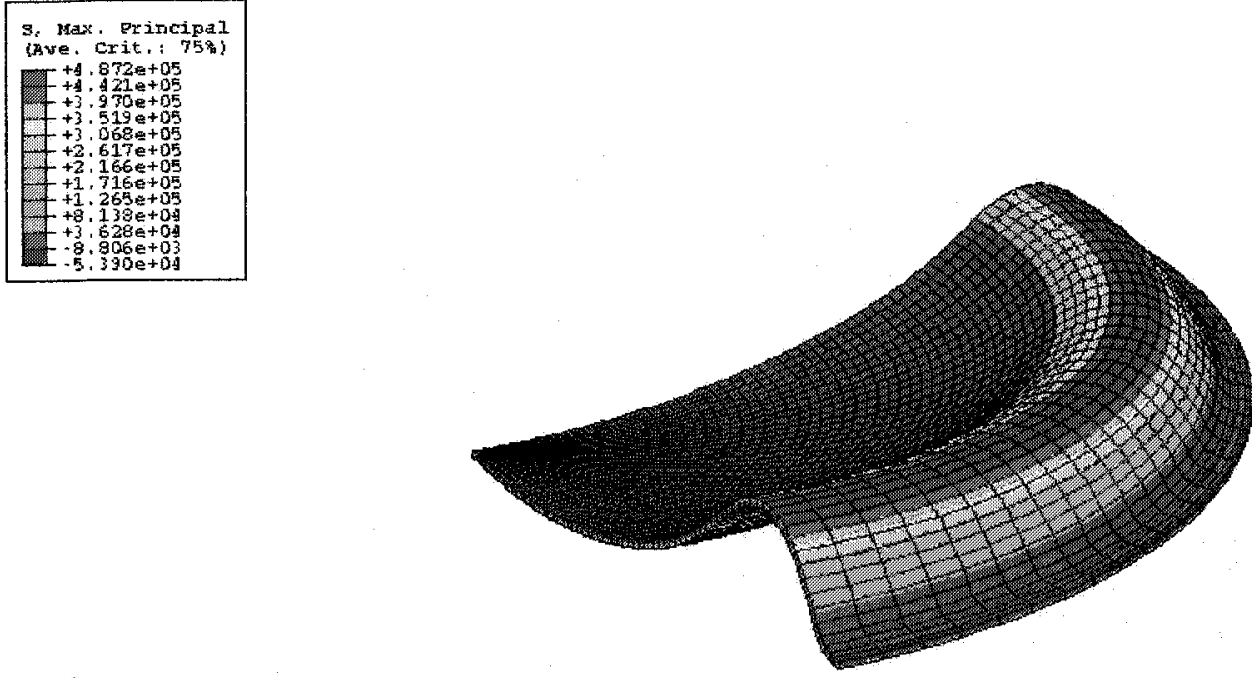

$L_{3}^{2}$

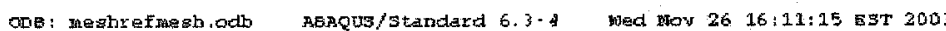

3tep: step-1

erimaty 14: Step Time $=0,871$

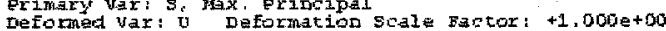

Figure 6.6: Deformation at $14 \mathrm{KPa}$ 

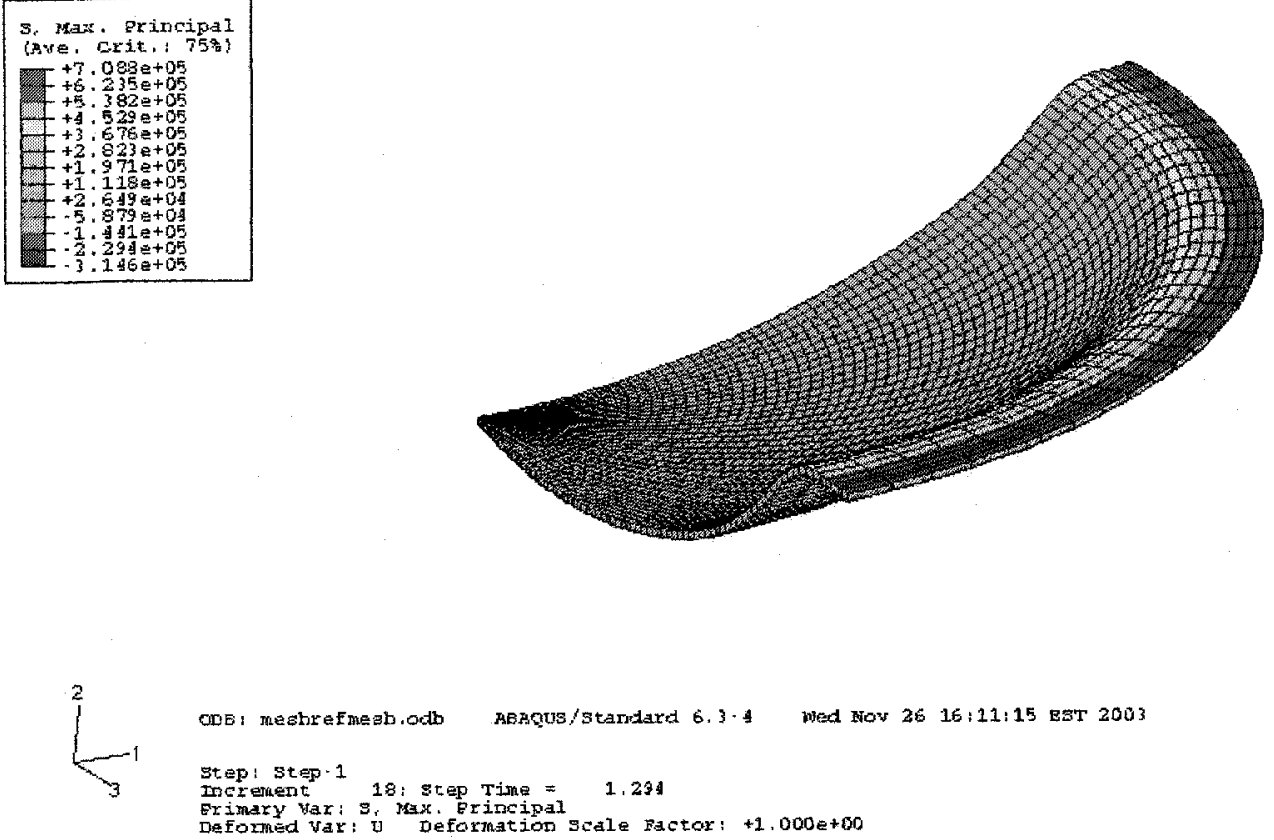

Figure 6.7: Deformation at $21 \mathrm{KPa}$
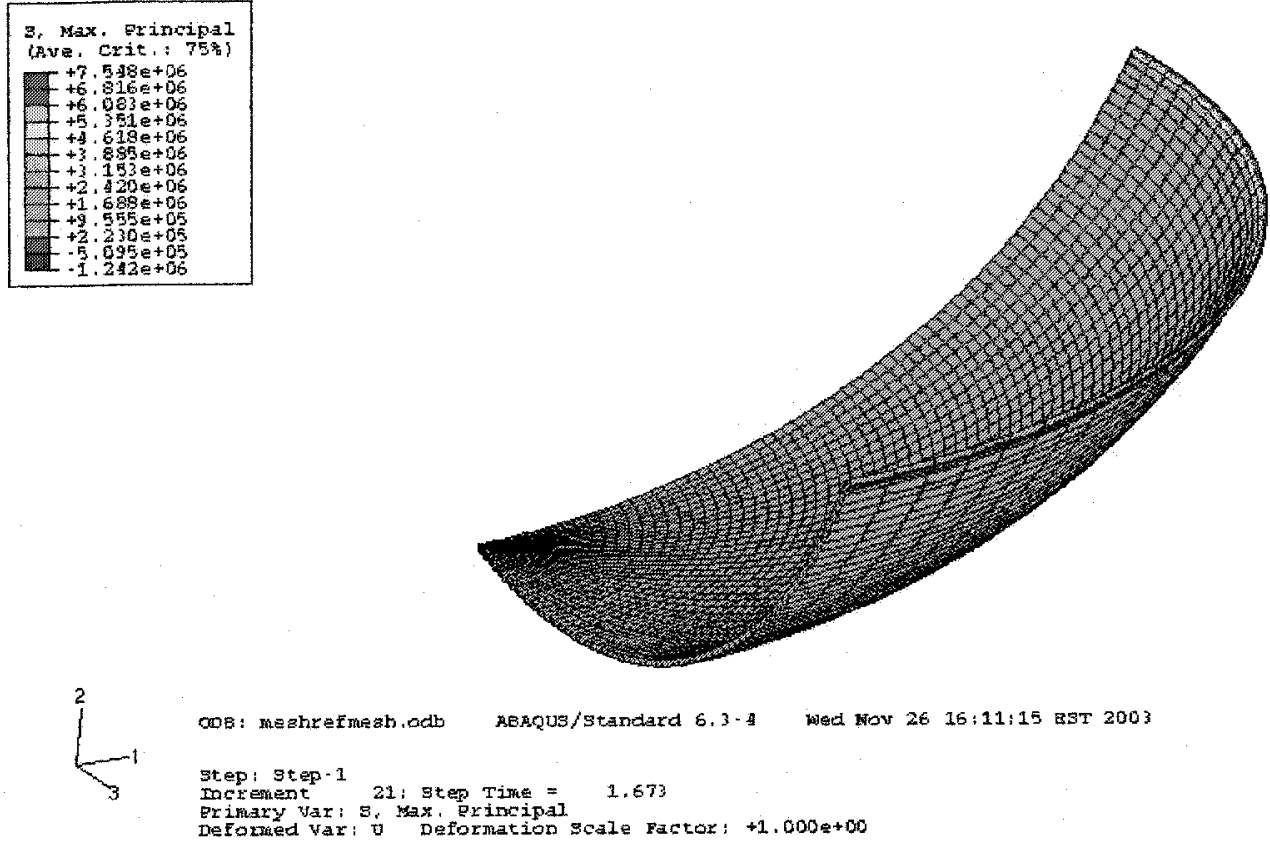

Figure 6.8: Deformation at $27 \mathrm{KPa}$ 


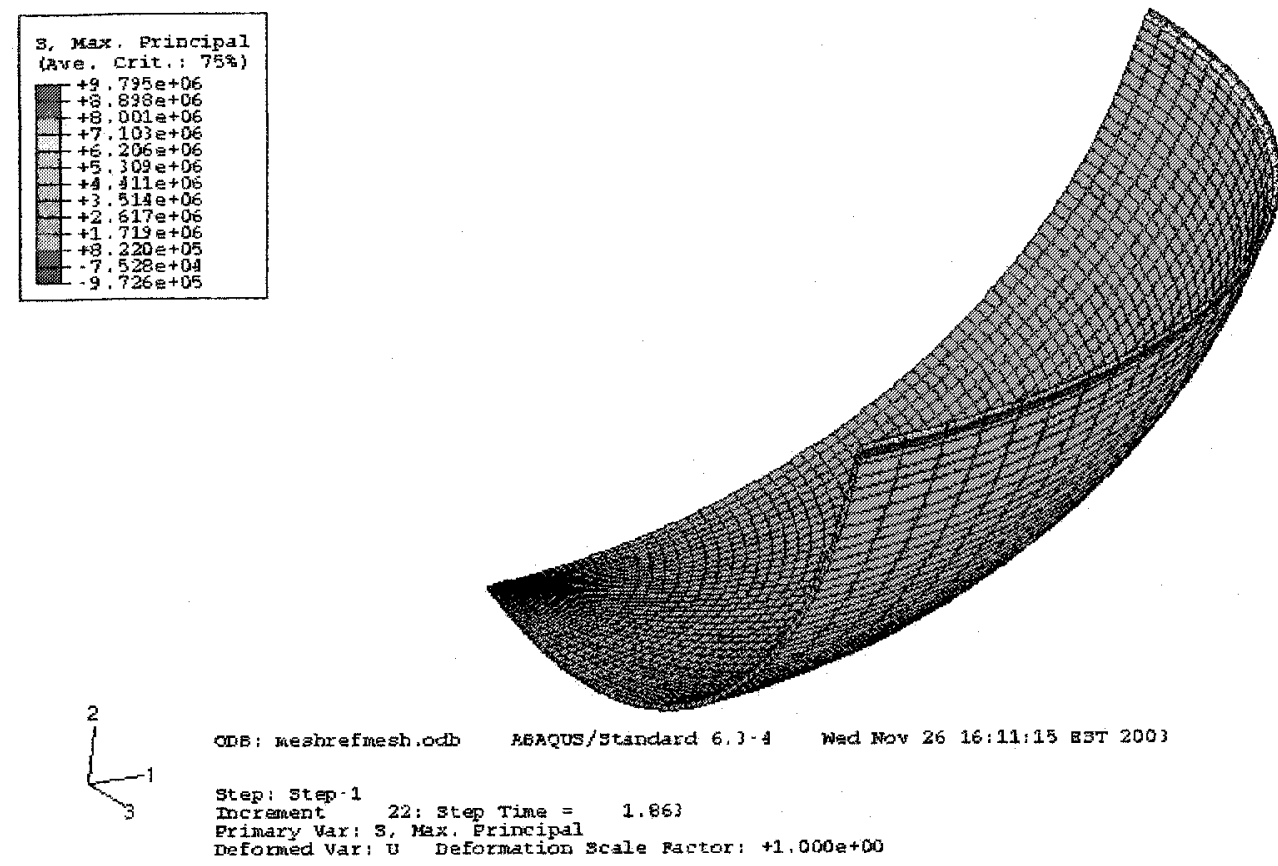

Figure 6.9: Deformation at 30KPa

diaphragm. When pressurization increases above $22 \mathrm{KPa}$, the strains are $>50 \%$ at the periphery.

The biaxial strains at the apex of the diaphragm appear to respond correctly to the coefficients obtained. After inverting the diaphragm, the simulation is stopped at an arbitrary value of $27 \mathrm{KPa}$. At this load, the strains at the apex of the deformed shape, are observed to be $11 \%$ and the stress is $1.65 \mathrm{MPa}$. This is in accordance with the biaxial stress-strain response shown in Figure 4.18.

There are a number of important findings from this analysis. The chosen pressure behind the diaphragm, required to invert the diaphragm is $255 \mathrm{mmHg}(30 \mathrm{KPa}$ ) and is enough to create significant deformation in the diaphragm. From an initial height of $2 \mathrm{~cm}$, the diaphragm can deform a further $4 \mathrm{~cm}$ if allowed room to inflate.

Large stress concentrations exist at the periphery of the diaphragm and are greatest when the diaphragm is inverted. This is expected as the molded shape will stretch to 
allow the deformation. Thus higher stress is experienced on one side of the diaphragm than another and it is clear that the least stressed side should face the most corrosive environment. The stresses in the folds as the depression propagates along the diaphragm are approximately $300 \mathrm{KPa}$. 


\section{Chapter 7}

\section{Discussion, Recommendations and}

\section{Conclusions}

This study showed that to adequately model the artificial heart diaphragm the material properties from biaxial and uniaxial strain must be determined and the finite element package must be qualified to handle meshing and with higher-order elements, non-linear large deformation analysis, and buckling analysis. This section begins with a review of the objectives of this thesis from Section 1.

\subsection{Discussion of Objectives}

Finite Element Analysis Referring back to the objectives of this thesis, the numerical error can be reduced with the proper application of finite element techniques. Firstly, a structured hexagonal mesh is applied, versus a tetrahedral mesh which would be too stiff for the analysis. Secondly, higher order elements are investigated and verified for volumetric locking.

The modified Riks method is used to overcome the dynamic snap through event. A 
solution is obtained using this option showing a dramatic reduction in stress after the event occurs. A new position for the diaphragm is established and pressurization continues.

Theory of Large Deformation Analysis The thesis also served to outline the theory of the ABAQUS software, by introducing the stress and strain measures for large deformation analysis.

Boundary Conditions Boundary conditions are an empirical problem and trying more than one boundary condition is essential in representing the problem. This study looked at the built-in boundary condition and it was found that for strains, $<100 \%$, observed in this study, the built-in boundary condition is acceptable.

Material Properties Modelling of an artificial heart diaphragm begins with the determination of material properties of the diaphragm. The analysis of large deformation hyperelastic materials is available and qualified, however, the material properties must be known to a high degree of accuracy or the output from the analysis is erroneous. In the inflation test the major sources of error are the pressure gauge precision and the magnification of the lens.

For rubber materials, curve fitting the data must be done carefully. It is proposed that in order to properly represent the uniaxial stress-strain curve at low strains, a specific range on the Mooney-Rivlin plot is selected. The most-linear range is not necessarily the most appropriate, as $<50 \%$ strain involves a different phenomenon in the morphology of polyurethane. Obtaining the correct curve fit in the strain range of interest is essential. The stress can be underestimated by as much as $25 \%$ as shown by obtaining coefficients from the "most linear region" of the Mooney-Rivlin plot.

It is also proposed that the full length of the stress-strain curve in biaxial strain need not be obtained. The data collected in this analysis is sufficient for our range of strains. 
The material evaluation also shows that the values generated from ABAQUS from the raw data are reasonable when evaluated against published data and the original stress-strain curves.

In reality, a hysteresis effect occurs that softens the material and lowers the stressstrain curve. This softening (stress reduction) can be as much as $20 \%$ when the material experiences a uniaxial strain of $50 \%$, as observed in Material \#2. This softening effect is further promoted by the introduction to the blood environment where even un-cycled samples exhibit an increase in ultimate strain as mentioned in Chapter 2.

Shear response is also a concern and information regarding pure shear in the sample is required. However, a stable response can be achieved with the biaxial and uniaxial data as a minimum requirement. In this study, it is proposed that the pure shear response is expected to fall between the biaxial and uniaxial stress-strain curves and thus the two tests conducted in this study represent limiting cases.

Polyurethane is a viscoelastic material although a time delayed response to strain is neglected as it is proposed that the strains are low during inversion. Furthermore, although the stress is high in stage 3 as defined in Chapter 2, the loading is instantaneous and the material is constrained from expanding too far by the housing.

\subsection{Recommendations}

It is expected that this study will be of interest to companies wishing to introduce computational analysis to the design process and thus, the following recommendations are made in order to improve the material testing and the model.

Material Testing Two major recommendations are made for the inflation test, both of which can improve the accuracy of the data. Firstly, the pressure gauge and control should be of higher accuracy. Secondly, this study uses a 10x magnification, however, it is possible 
to achieve $20 \mathrm{x}$ magnification on the optical comparator thus improving the accuracy of the data.

Benchmarking It was found that the Williams model was inadequate to describe the inflation of the thin polyurethane disk with high Mooney-Rivlin coefficients. It is recommended that a more suitable model is obtained and/or that this model is analysed for its relevance.

It is possible to assess the ability of the ABAQUS analysis methods and modelling by analyzing a flat disk model of the same diameter as the test sample in the inflation test and the coefficients generated from the raw data. The approximate deflection at centre can be determined from pressurizing one side of the plate.

Finally, with the circular disk model, it is recommended that a diaphragm be cast of the dimension in this study and a static test be conducted to determine the location of the pole of the dome shape at varying pressures. This will further assist in checking the validity of the model and boundary conditions.

Material Sensitivity Analysis Once the above recommendations are addressed, it may be of interest to investigate the impact of adjusting the stress-strain curve by reducing the values equally by $20 \%$ to observe the response of the diaphragm. In other words, inducing softening in the material and observing the effect in the final component design. These adjustments to material properties can also affect the stress and strain response and the thickness reduction. These are also of interest to the designer.

Motion of the Diaphragm Jin and Clark also investigated the motion of the diaphragm in different physical orientations of the VAD. If the VAD is oriented vertically, for example, when the VAD fills, the diaphragm is observed to begin motion closest to the inlet of the blood chamber. A depression appears in one region of the diaphragm and 

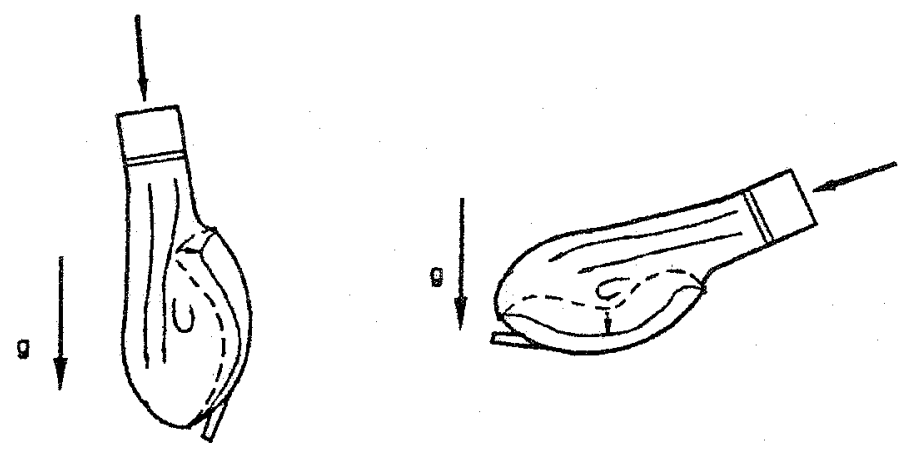

Figure 7.1: Vertical(left) and horizontal(right) orientation of VAD and effect on diaphragm motion [55].

eventually the diaphragm begins to flip over. When the VAD is oriented horizontally, no region of the diaphragm is said to move sooner than another, and the response to pressurization is as obtained in this study. This simulation shows that the center is depressed first and the depression grows until the diaphragm flips over. Thus, it can be said that this study is the condition VAD perpendicular to the gravity vector or when the patient is lying down.

The fluid motion and the hydrostatic effect on the diaphragm motion was presented by Jin and Clark [55] and are shown in Figure 7.1. Thus, for a more complete model, a fluid-solid interaction is proposed and the analysis be performed dynamically versus statically.

\subsection{Conclusions}

Finite Element Analysis, and ABAQUS in particular, is capable of modelling the inflation of rubber membranes and thus is applicable to diaphragm analysis.

The Mooney-Rivlin coefficients obtained for Material \#1, are similar to Biomer.

Second order elements with reduced integration, C3D20RH, exhibit volumetric locking, however, they can be used in inflation of rubber disk analysis exhibiting strains $<50 \%$. 
The built-in boundary condition as defined in this study, is adequate for the inversion of the diaphragm.

The Mooney-Rivlin curve fit can be applied to polyurethanes, such as Material \#2 or Material \#1, provided that raw data are collected within the range of interest as different phenomomen occurs at strains $<50 \%$. 


\section{Bibliography}

[1] J.D. Bronzino, editor. The Biomedical Engineering Handbook. CRC Press, 1995.

[2] Worldheart Corporation. WEB cast, heartsaver VAD release. Internet: www.worldheart.com, February 2003.

[3] A. El-Banayosy, L. Arusoglu, L. Kizner, G. Tenderich, K. Minami, K. Inoue, and R. Korfer. Novacor left ventricular assist system vs. Heartmate vented electric left ventricular assist system as a long-term mechanical circulatory support device in bridging patients: A prospective study. Journal of Thoracic and Cardiovascular Surgery, pages 581-587, March 2000.

[4] R. Sorelle. Assist pumps give diseased organ rest, and chance to recover. Houston Chronicle, 1997.

[5] D. Y. Loisance. Long-term mechanical circulatory support with the wearable Novacor left ventricular assist system. European Journal of Cardio-thoracic surgery, 18:220224,2000 .

[6] S. Murali. Mechanical circulatory support with the novacor lvas: World-wide clinical results. Thoracic Cardiovascular Surgery, 47:321-325, 1999. 
[7] F. de Vivo, L. S. De Santo, C. Maiello, C. Marra, and M. Cotrufo. Novacor left ventricular assist device: Present Experience. The International Journal of Artificial Organs, 22(1):11-13, 1999.

[8] S. Chu and R. Hsu. Current status of artificial hearts and ventricular assist devices. Journal of the Formosan Medical Association, 99(2):79 85, 2000.

[9] P. A. Dew, G. M. Pantalos, J. W. Holfert, K. D. Murray, and D. B. Olsen. Mechanical failures of the pneumatic Utah-100 and Jarvik total artificial hearts. Transactions of the American Society of Artificial Internal Organs, 35:697-699, 1989.

[10] Gayle Ehrenman. A whole new heart. Mechanical Engineering, 125(8):51-53, August 2003.

[11] K. Minami, A. El-Banayosy, A. Sezai, L. Arusoglu, P. Sarnowsky, O. Fey, and R. Korfer. Morbidity and outcome after mechanical ventricular support using throatec, novacor and heartmate for bridging to heart transplantation. Artificial Organs, 24(6):421426,2000 .

[12] H. J. Kim and R. S. Benson. The effects of soft segment structure on the fatigue crack propagation of model polyurethanes. Bio-Medical Materials and Engineering, $4(3): 171-185,1994$.

[13] A. Ertas and J. C. Jones. The Engineering Design Process. John Wiley and Sons Inc., New York, 2nd edition, 1996.

[14] B. Haridas and C. A. Haynes. Predictive analysis at the forefront of medical product development. Medical Device and Diagnostic Industry, 1999.

[15] V L. Poirier. The LVAD: A case study. The Bridge Volume, 27(4), 1997.

[16] J. T. Oden. Finite Elements of Non-Linear Continua. McGraw Hill, New York, 1972. 
[17] L.J. Hart-Smith and J.D.C. Crisp. Large elastic deformations of thin rubber membranes. Int.J.Eng.Sci., 5(1):1-24, 1967.

[18] W. Jin and C. Clark. Pressure development within a sac-type pneumatically driven ventricular assist device. Journal of Biomechanics, 27(11):1319-1329, 1994.

[19] A. Pigeard. Artificial hearts. http://membres.lycos.fr/chezalex/, 2003.

[20] A. Sezai et al. Major organ function under mechanical support: Comparative studies of pulsatile and nonpulsatile circulation. Artificial Organs, 23(3):280-285, 1999.

[21] F. Zartnack, K. Affeld, and E.S. Bucherl. Fatigue problems with artificial blood pumps. In Abstracts for General Papers 2nd International Symposium for Artficial Internal Organs, page 149, Tokyo, Japan, August 26-28 1977. International Society of Artificial Organs.

[22] A. Ionita. Finite Element Analysis of the Deformation of a Rubber Diaphragm. PhD, Virginia Polytechnic and State University Engineering Science and Mechanics, Virginia, February 2001.

[23] B. Knierbien, K. Schleede, Ch. Sturm, H. Reul, B.J. Messmer, and G. Rau. Stress simulation in the PUR diaphragm of a blood pump. Kunstoffe German Plastics, $78: 24-25,1988$.

[24] Didier Chatel, L. Delamare, P. Dang, D. Lebouvier, and F. Trocherie. A biomechanical double sac (Pericardium-Pebax) for specially shaped artificial ventricles: A computerized study to evaluate its mechanical and volumetric properties. International Society of Artificial Organs, 1997. 
[25] A. G. McColgan. Design of a durability test rig for testing of an elastomeric blood pump diaphragm used in a ventricular assist device. M.Eng., Carleton University: Institute of Aerospace and Mechanical Engineering, Ottawa, Ontario, June 2001.

[26] C. P. Sharma and M. Szycher. Blood Compatible Materials and Devices: Perspectives Towards the 21st Century. Technomic Publishing Company, Inc., Pennsylvania, U.S.A, 1991.

[27] C. R. McMillin. Characterization of elastomers for use in circulatory assist devices. ACS Symposium Series, 182nd Meeting of the American Chemical Society, 193:533$548,1981$.

[28] B. O'Conner, G. M. Bernacca, I. Straub, and D. J. Wheatley. Mechanical testing of flexible polyurethanes: Candidate selection for a prosthetic heart valve. Transactions from the Sixth World Biomaterials Congress, Society for Biomaterials, 2000.

[29] S. Doshi. Fatigue testing of biocompatible polymers. Undergraduate, B.Sc., University of Ottawa, Ottawa, Ontario, June 2000.

[30] K. Hayashi, H. Takano, T. Matsuda, and M. Umezu. Effects of implantation on the mechanical properties of the polyurehtane diaphragm of left ventricular assist devices. Biomaterials, 6:82-88, March 1985.

[31] K. Hayashi, H. Takano, T. Matsuda, M. Umezu, Y. Taenaka, and T. Nakamura. Mechanical stability of elastomeric polymers for blood pump applications. Journal of Biomedical Materials Research, 19:179-193, 1985.

[32] F. Orang, C. J. G. Plummer, and H. H. Kausch. Effects of processing conditions and in vitro ageing on the physical properties of biomer. Biomaterials, Elsevier Science, $17: 485-490,1996$. 
[33] J. L. Kardos, A.P. Bhate, and K. P. Gadkaree. Durability prediction and testing of elastomeric biomaterials. Advances in Biomaterials, pages 271-277, 1987.

[34] A. P. Bhate and J. L. Kardos. A novel technique for the determination of high frequency equibiaxial stress-deformation behaviour of viscoelastic elastomers. Polymer Engineering and Science, 24(11):862-868, August 1984.

[35] W. D. Callister. Materials Science and Engineering: An Introduction. Wiley, New York, 6 edition, 2003.

[36] M. Munro. Course notes for MCG2365, Materials 2. University of Ottawa, 1997.

[37] American Plastics Council. Rubbers. Internet: www.americanplasticscouncil.org, 2003.

[38] Alan N. Gent, editor. Engineering with Rubber: How to Design Rubber Components. Oxford University Press, New York, 1992.

[39] G. A. Abraham, P. M. Frontini, and T. R. Cuadrado. Physical and mechanical behavior of sterilized biomedical segmented polyurethanes. Journal of Applied Polymer Science, 65(6):1193, 1997.

[40] David Shaw. Constitutive models cause problems for rubber FEA. European Rubber Journal, 181(10):30, October 1999.

[41] G. E. Mase. Schaum's Outlines: Continuum Mechanics. McGraw Hill, New York, 1970 .

[42] ABAQUS, Inc. Section 10.5.1 Hyperelastic Behaviour, ABAQUS Manual, 6.3 edition.

[43] Mario M. Attard and Giles W. Hunt. Hyperelastic constitutive modeling under finite strain. June 2003. 
[44] L.R.G. Treloar. Stress-strain data for vulcanized rubber under various types of deformation. Transactions of the Faraday Society, 40:59-70, February 1944.

[45] Kurt Miller. Testing elastomers for hyperelastic material models in finite element analysis. Company Product Literature, July 2000.

[46] A. Makino, W. R. Hamburgen, and J. S. Fitch. Fluoroelastomer pressure pad design for microelectronic applications. Research Report 93/7, Digital Western Research Laboratory, Palo Alto, California, November 1993.

[47] MSC.Software Corporation. Non-linear finite element analysis of elastomers. Company Product Literature, 2000.

[48] ABAQUS, Inc. Section 3.2.3, Hybrid Element Solid Element Formulation, ABAQUS Manual, 6.3 edition.

[49] ABAQUS, Inc. Section 2.2, Non-Linear Solution Methods, ABAQUS Manual, 6.3 edition.

[50] ABAQUS, Inc. Modified Riks Procedure: Reference Shelf, ABAQUS Manual, 6.3 edition.

[51] E. D. George. The integration of analysis and testing for the simulation of the response of hyperlastic materials. Finite Elements in Analysis and Design, 4:19-42, 1988.

[52] T. J. R. Hughes and E. Carnoy. Non-linear finite element shell formulation accounting for large membrane strains. AMD, pages 193-208, 1981.

[53] B. Haggblad and J. A. Sundberg. Large strain solutions of rubber components. Computers and Structures, 17(5):835-843, 1983.

[54] J. G. Williams. Stress Analysis of Polymers. Longman Group LTD., London, 1973. 
[55] W. Jin and C. Clark. Experimental investigation of the motions of the pumping diaphragm within a sac-type pneumatically driven ventricular assist device. Journal of Biomechanics, 27(1):43-55, 1994. 


\section{Appendix A}

\section{MathCAD Metal Plate Simulation}




\section{Analysis of Metal Thin Plate and Abaqus FEA, 20 Node Brick}

$$
\begin{aligned}
& \begin{array}{l}
p=30000 \\
\mathrm{x}=0.05 \\
\mathrm{v}=0.29
\end{array} \quad \mathrm{I}=\left(\begin{array}{l}
0.01 \\
0.02 \\
0.03 \\
0.04 \\
0.05
\end{array}\right) \\
& M r:=\frac{p}{16} \cdot\left[a^{2}(1+v)-r^{2} \cdot(3+v)\right] \\
& M=\left(\begin{array}{c}
5.43 E+000 \\
3579375 \mathrm{E}+0000 \\
495 \mathrm{E}-003 \\
-3.823125 \mathrm{E}+000 \\
-9.375 \mathrm{E}+000
\end{array}\right) \\
& M \theta:=\frac{p}{16}\left[a^{2}(1+v)-t^{2}(1+3 v)\right] \\
& M \theta=\left(\begin{array}{c}
5.69625 E+000 \\
4.644375 E+000 \\
2.89125 E+000 \\
436.875 E-0003 \\
-2.71875 E+000
\end{array}\right) \\
& z:=0.00025 \\
& h:=0.0005 \\
& \text { or }:=12 \cdot \mathrm{Mr} \cdot \frac{\mathrm{z}}{\mathrm{h}^{3}} \\
& \nabla \theta:=12 \cdot M \theta \cdot \frac{z}{h^{3}} \\
& \sigma r=\left(\begin{array}{c}
130.32 \mathrm{E}+006 \\
85.905 \mathrm{E}+006 \\
11.88 \mathrm{E}+006 \\
-91.755 \mathrm{E}+006 \\
-225 \mathrm{E}+006
\end{array}\right) \\
& \sigma \theta=\left(\begin{array}{c}
136.71 \mathrm{E}+006 \\
111.465 \mathrm{E}+006 \\
69.39 \mathrm{E}+006 \\
10.435 \mathrm{E}+006 \\
-65.25 \mathrm{E}+006
\end{array}\right) \quad D:=\mathrm{E} \frac{\left(\mathrm{h}^{3}\right)}{\left[12\left(1-\mathrm{v}^{2}\right)\right]} \\
& w=1.288 \mathrm{E}-003
\end{aligned}
$$


Appendix B

Williams Model Spreadsheet 


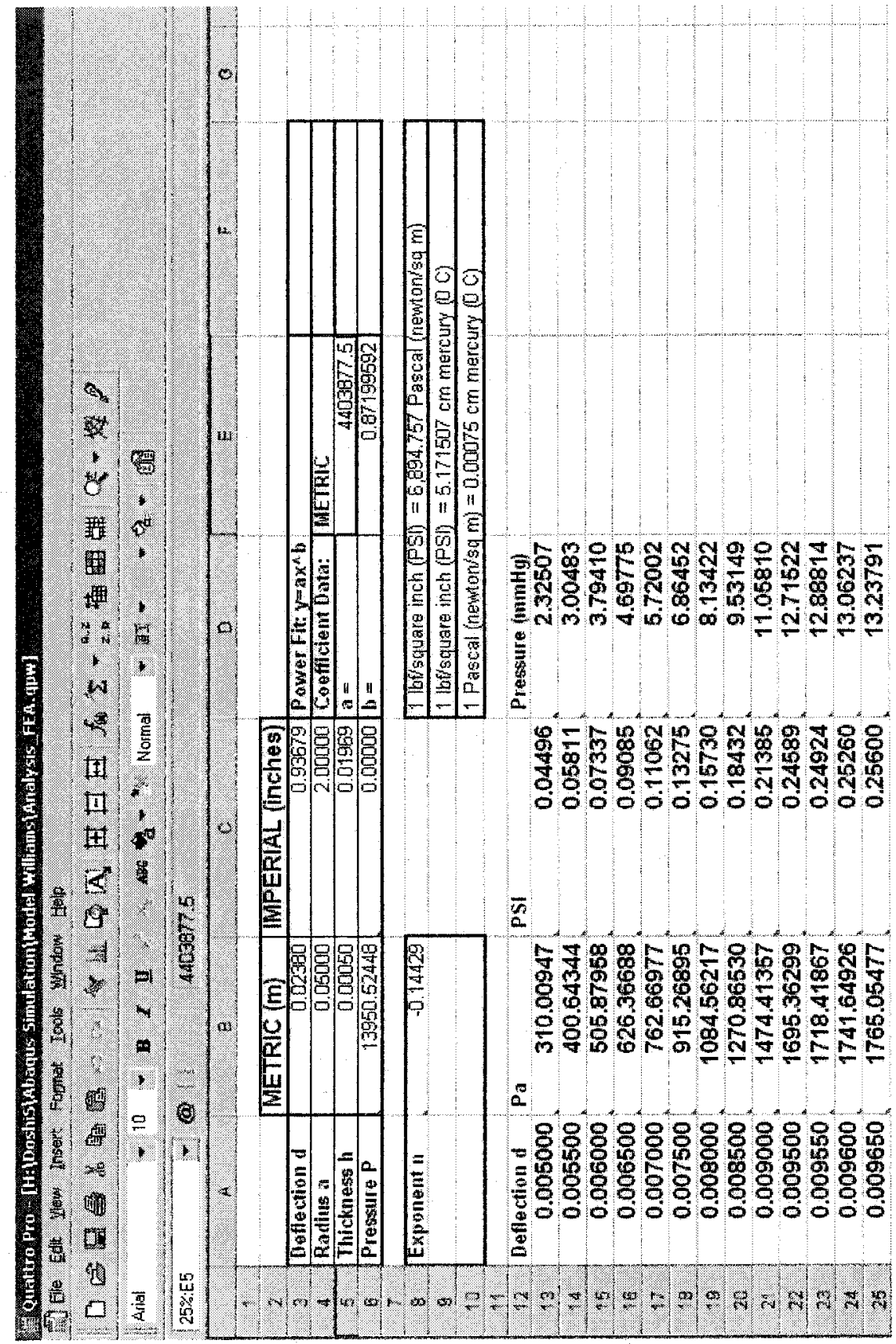




\section{Appendix C}

\section{CAE and Input Files}

Three ABAQUS/Standard input files can be found in the attached CD. Input files can be imported into ABAQUS/CAE and unless noted below, the mesh, loads, boundary conditions and material properties can be changed by the user. CAE files can be opened in ABAQUS/CAE as they are but require that that the JNL file is in the same folder as the CAE file.

thindisk.cae, thindisk.jnl 3D model of thindisk used in metal analysis and rubber analysis.

axisymmetric.inp This model contains the pinned boundary condition for the axisymmetric model. CA8RH elements and geometry are set as in the analysis run in this study. This file contains the final meshed part only.

diaphragm.cae, diaphragm.jnl These files contain the final diaphragm model of this study. Once again, the mesh can be generated using ABAQUS/CAE. An example mesh part exists for the C3D20RH elements. 